\title{
MATCHED FILTER BOUNDS FOR FAST FADING RICIAN CHANNELS
}

\author{
by
}

\section{VAIBHAV DINESH}

B. E. (Electronics and Communications), Guru Jambheshwar University, India, 1998

A THESIS SUBMITTED IN PARTIAL FULFILLMENT OF

THE REQUIREMENTS FOR THE DEGREE OF

MASTER OF APPLIED SCIENCE

in

THE FACULTY OF GRADUATE STUDIES

DEPARTMENT OF ELECTRICAL AND COMPUTER ENGINEERING

We accept this thesis as conforming

to the required standard

THE UNIVERSITY OF BRITISH COLUMBIA

March 2002

(C) Vaibhav Dinesh, 2002 
In presenting this thesis in partial fulfilment of the requirements for an advanced degree at the University of British Columbia, I agree that the Library shall make it freely available for reference and study. I further agree that permission for extensive copying of this thesis for scholarly purposes may be granted by the head of my department or by his or her representatives. It is understood that copying or publication of this thesis for financial gain shall not be allowed without my written permission.

Department of ELECTRICAL AND COMPUTER ENGINEERING

The University of British Columbia

Vancouver, Canada

Date O8 MARCH 2002. 


\begin{abstract}
A new matched filter bound (MFB) for a dispersive Rician fading channel with unrestricted normalized Doppler rate is presented. Analytical expressions are derived for BPSK modulation.
\end{abstract}

The channel model is based on the standard linear time varying filter. The BER performance, in general, is found to improve with an increase in the fading rate due to the inherent diversity present within a single pulse. The shape of the transmitted pulse is shown to affect the BER in fast fading conditions. It has been found that the specular component improves the BER at lower fading rates; for very high fading rates, the implicit diversity effect becomes dominant in diminishing the effect of fading.

Discrete (two and three beam) models are assumed for the dispersive channel. The error bounds for the three-beam model are derived as a function of the inter-beam delay and correlation parameters. In general, the delay spread was found to result in lowering the BER for both the slow and fast fading cases when the fading in the beams is uncorrelated. In the two-beam frequency selective case, the BER is very sensitive to the degree of beam overlap.

Finally, assuming errors in estimating the channel fading waveform, the BER for a nonideal receive filter is obtained. It was observed that, for the specified model, the system degradation is higher for phase mismatches than for amplitude mismatches for a given error variance. 


\section{Table of Contents}

Abstract $\quad$ ii

List of Figures $\quad$ v

Glossary vii

Acknowledgment $\quad$ xi

$\begin{array}{llr}\text { Chapter } 1 & \text { Introduction } & 1\end{array}$

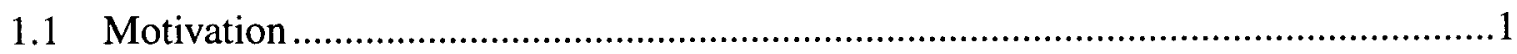

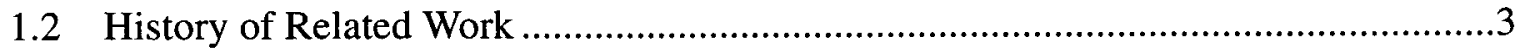

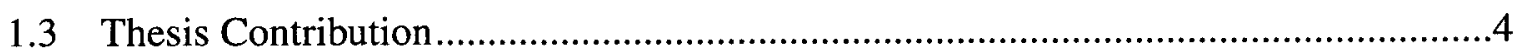

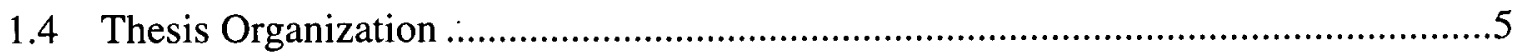

$\begin{array}{lll}\text { Chapter } 2 & \text { The Wireless Channel } & 6\end{array}$

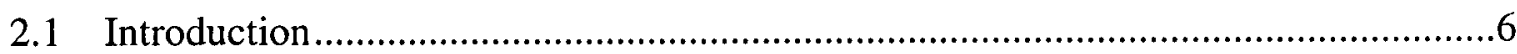

2.2 Signal Propagation in a Wireless Channel .....................................................6

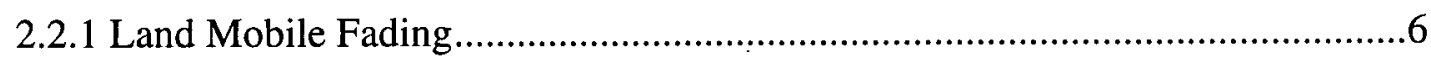

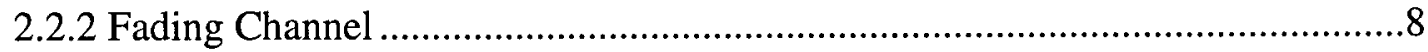

2.3 Channel Model and it's statistical Characterization..........................................11

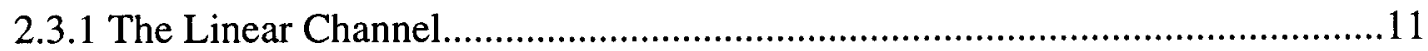

2.3.2 RF Spectrum and Autocorrelation of the Fading Process...........................17

$\begin{array}{llr}\text { Chapter } 3 & \text { Derivation of the MFB's } & 20\end{array}$

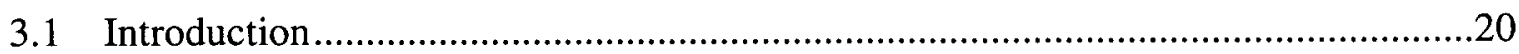

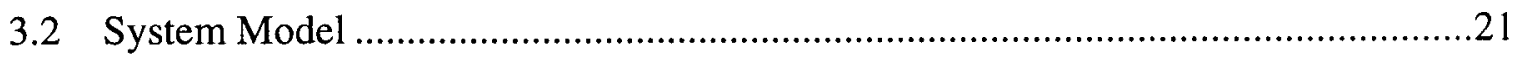

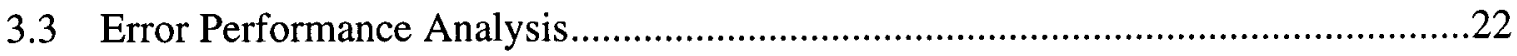

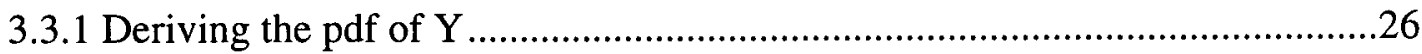




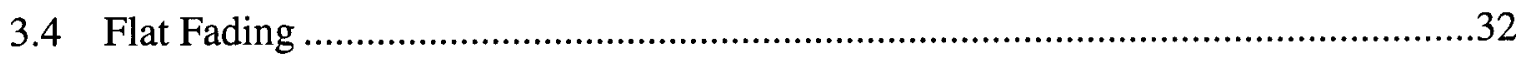

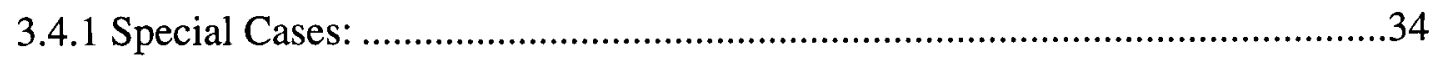

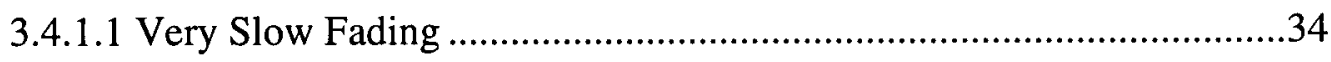

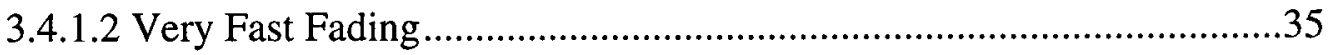

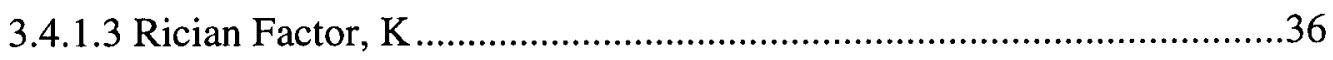

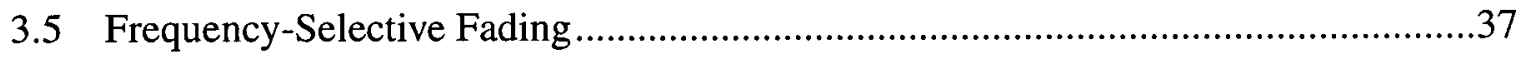

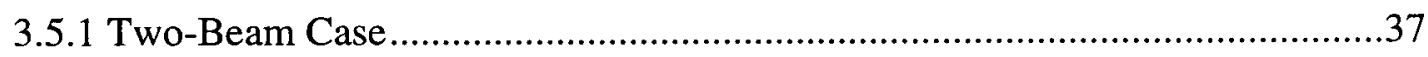

3.5.2 Three-Beam Case ........................................................................... 41

$\begin{array}{lll}\text { Chapter } 4 \text { Numerical Results } & 45\end{array}$

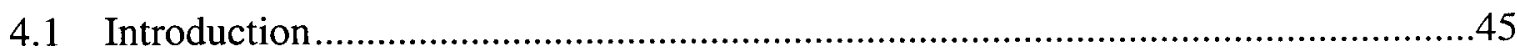

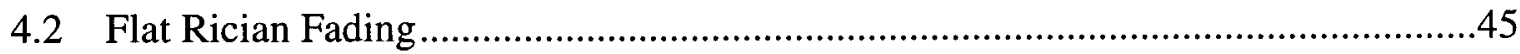

4.3 Frequency-Selective Rician Fading ......................................................50

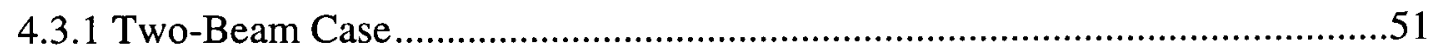

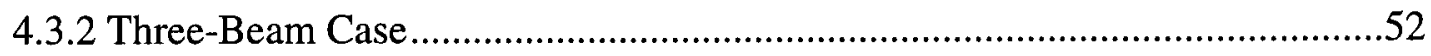

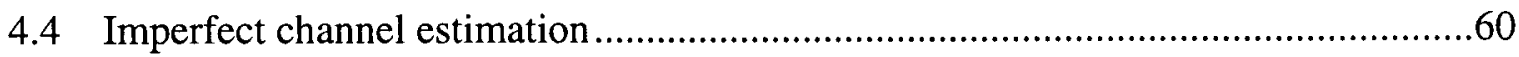

Chapter 5 Conclusions and Suggestions for Future Work 65

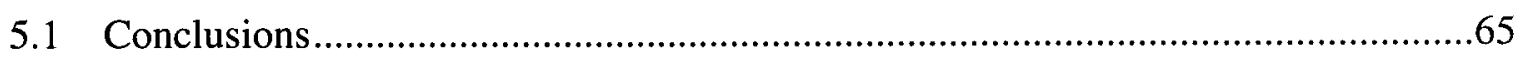

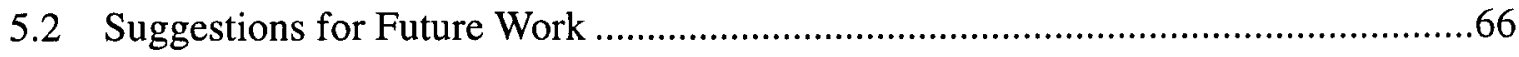

Appendix A : MFB (slow fading $0.0<f_{N}<0.01$ ) 68

Appendix B : MFB (fast fading $f_{N} \rightarrow \infty$ )

$\begin{array}{ll}\text { Appendix C : Solving the Integral } & 74\end{array}$

Appendix D : Calculating the Errors in Amplitude and Phase estimation 77

$\begin{array}{ll}\text { Bibliography } & 79\end{array}$ 


\section{List of Figures}

Figure 1.1 Received signal corresponding to slow and fast fading channels ..............2

Figure 2.1 Continuously Dispersive and Discrete Path channel outputs.....................9

Figure 2.2 $\quad$ Linear System model ................................................................. 12

Figure 2.3 3-D view of the continuous-time impulse response, $|z(t, \tau)| \ldots \ldots \ldots \ldots \ldots \ldots . . .14$

Figure 2.4 3-D view of the discrete-time impulse response, $|z(t, \tau)| \ldots \ldots \ldots \ldots \ldots \ldots \ldots . . . .17$

Figure 2.5 Power Spectrum of an unmodulated CW carrier ..................................19

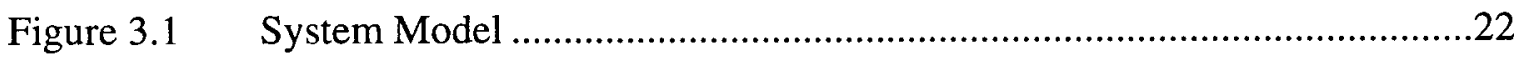

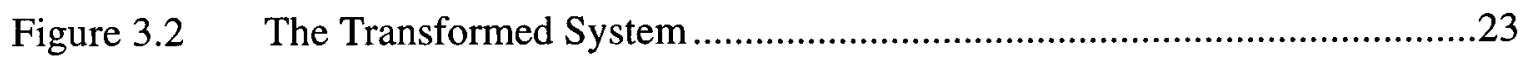

Figure 4.1 BER as a function of $f_{N}$ for the Rician channel, $K_{d B}=0 \mathrm{~dB} \ldots \ldots \ldots \ldots . . .46$

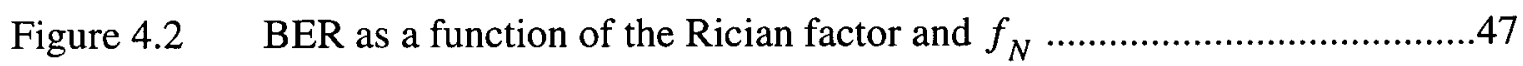

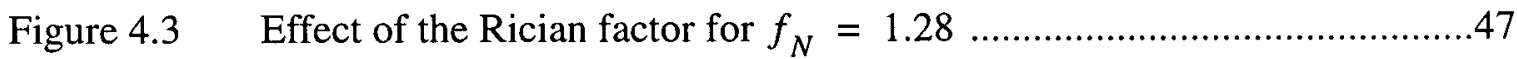

Figure 4.4 Effect of pulse shape in fast fading Rician channel, $K_{d B}=0 \mathrm{~dB} \ldots \ldots \ldots . .48$

Figure 4.5 Channel averaging ability of different pulse shapes ..............................49

Figure 4.6 Effect of the roll-off factor on the system performance, $K_{d B}=0 \mathrm{~dB} \ldots . .50$

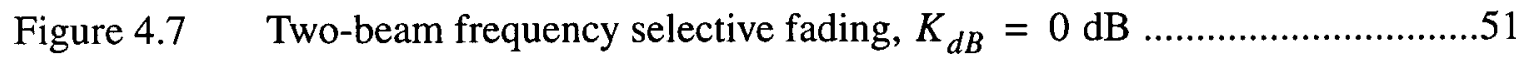

Figure 4.8 Three-beam frequency selective fast fading, $f_{N}=0.64$ and $\sigma=0 \ldots .52$

Figure 4.9 Three-beam frequency selective very-slow fading, $\sigma=0 \ldots \ldots \ldots \ldots \ldots \ldots \ldots . .53$

Figure 4.10 Three-beam frequency selective fast fading, $f_{N}=0.64$ and $\sigma>0 \ldots \ldots .54$

Figure 4.11 Three-beam frequency selective very-slow fading, $\sigma>0 \ldots \ldots \ldots \ldots \ldots \ldots \ldots . . .55$

Figure 4.12 Three-beam fast fading, $K_{d B}=0 \mathrm{~dB}$ and $f_{N}=0.64 \ldots \ldots \ldots \ldots \ldots \ldots \ldots . . .56$

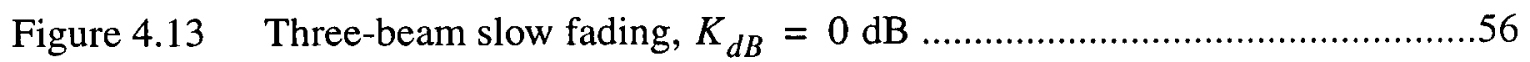

Figure 4.14 Three-beam fast fading as a function of $K ;[\sigma ; \rho]:[0.0 ; 0.0],[1.0 ; 1.0] \ldots \ldots .57$ 
Figure 4.15 Three-beam fast fading as a function of $K ;[\sigma ; \rho]:[0.0 ; 1.0],[1.0 ; 0.0] \ldots \ldots . .58$

Figure 4.16 Three-beam slow fading as a function of $K ;[\sigma ; \rho]:[0.0 ; 0.0],[1.0 ; 1.0] \ldots .58$

Figure 4.17 Three-beam slow fading as a function of $K ;[\sigma ; \rho]:[0.0 ; 1.0],[1.0 ; 0.0] \ldots .59$

Figure 4.18 Performance degradation in a flat fading Rician channel ......................60

Figure 4.19 Performance degradation in a flat fading Rayleigh channel ...................61

Figure 4.20 Effect of mis-match in phase on the BER in a Rician channel ................62

Figure 4.21 Effect of mis-match in phase on the BER in a Rayleigh channel ............62

Figure 4.22' Effect of mis-match in amplitude on the BER in a Rician channel ..........63

Figure 4.23 Effect of mis-match in amplitude on the BER in a Rayleigh channel ......64

Figure D.1 Resolution of the fading sample with/without error .............................77 


\section{Glossary}

\section{Acronyms}

$\begin{array}{ll}\text { ASK } & \text { Amplitude Shift Keying } \\ \text { AWGN } & \text { Additive White Gaussian Noise } \\ \text { BER } & \text { Bit Error Rate } \\ \text { BFSK } & \text { Binary Frequency Shift Keying } \\ \text { BPSK } & \text { Binary Phase Shift Keying } \\ \text { BS } & \text { Base Station } \\ \text { CD } & \text { Continuously Dispersive } \\ \text { CW } & \text { Continuous Wave } \\ \text { DP } & \text { Discrete Path } \\ \text { IFD } & \text { Intrinsic Frequency Diversity } \\ \text { ISI } & \text { Inter-Symbol Interference } \\ \text { LOS } & \text { Line Of Sight } \\ \text { MFB } & \text { Matched Filter Bound } \\ \text { MS } & \text { Mobile Station } \\ \text { MSE } & \text { Mean Square Error } \\ \text { OFDM } & \text { Orthogonal Frequency Division Multiplexing } \\ \text { PAM } & \text { Pulse Amplitude Modulation } \\ \text { PSK } & \text { Phase Shift Keying } \\ \text { RRC } & \text { Root Raised Cosine } \\ \text { SNR } & \text { Signal to Noise Ratio } \\ & \end{array}$




\section{Symbols}

$a$

A

$c$

$D_{r m s}$

$E_{b}$

$f_{c}$

$\Delta f_{c}$

$f_{d}$

$f_{N}$

$f(t)$

$f_{Y}(y)$

$h(t)$

$J_{0}($.

$K$

$K_{d B}$

$\mathrm{N}$

$N$

$N_{0}$

$n(t)$

$P_{b}$

$p(t)$

$Q(v, w)$

$r(t)$

$r_{c d}(t)$

$r_{d p}(t)$

$R_{f}\left(t_{l}, t_{2}\right)$

$r_{\text {fad }}(t)$

$r_{s}(t)$

$S(f)$ roll-off factor of the root raised cosine pulse

transmitted signal amplitude

speed of light

rms delay spread

bit energy

carrier frequency

Coherence bandwidth

maximum Doppler frequency

maximum normalized Doppler rate

received pulse shape corresponding to the diffused channel

pdf of $Y$

impulse response of the receive filter

zeroth order Bessel function of the first kind

Rician factor of the channel

Rician factor (in dB)

total number of paths in the discrete channel model

noise component at the matched filter output

power spectral density of AWGN

AWGN noise

probability of bit error

transmitted pulse shape

Marcum's $Q$-function

received (faded and noisy) signal

received signal (for a $\mathrm{CD}$ channel)

received signal (for a DP channel)

autocorrelation of the process $f(t)$

faded signal at the channel output

received signal without noise (for the DP channel)

power spectrum density of the Rayleigh fading process 


$\begin{array}{ll}s(t) & \text { transmitted signal } \\ s_{f a d}(t) & \text { received pulse shape } \\ T & \text { received signal duration } \\ \Delta t & \text { sampling interval } \\ \Delta t_{c} & \text { Coherence time } \\ T_{M} & \text { delay spread of the channel } \\ T_{p} & \text { pulse duration } \\ v & \text { maximum velocity of MS } \\ \mathrm{W}_{\lambda, v}(z) & \text { Whittaker function } \\ X & \text { matched filter output after sampling } \\ x(t) & \text { matched filter output } \\ Y & \text { signal component at the matched filter output } \\ z(t, \tau) & \text { impulse response of a linear channel (generalized notation) } \\ z_{c d}(t, \tau) & \text { impulse response of a CD channel } \\ z_{r a y}(t, \tau) & \text { impulse response of the diffused channel } \\ z_{r i c e}(t, \tau) & \text { impulse response of the Rician channel } \\ z_{n}(t) & \text { complex fading process corresponding to the } n \text {th beam }\end{array}$




\section{Greek Symbols}

\begin{tabular}{|c|c|}
\hline$\alpha^{2}$ & diffused beam strength \\
\hline$\alpha_{0}$ & attenuation corresponding to the direct/specular path \\
\hline$\alpha_{n}$ & strength of the $n$th faded beam \\
\hline$\alpha_{n}(t)$ & $n$th path attenuation \\
\hline$\Psi(v)$ & characteristic function \\
\hline$\delta(t)$ & delta function \\
\hline$\Gamma(z)$ & Gamma function \\
\hline$\varphi_{n}(t)$ & $n$th path phase/ eigen-function \\
\hline$\phi(t)$ & autocorrelation function of the Rayleigh fading process \\
\hline$\phi_{z_{1} z_{2}}(\tau)$ & cross-correlation function of the fading processes \\
\hline$\lambda$ & signal wavelength/ eigenvalue \\
\hline$\rho$ & normalized correlation coefficient of the fading processes \\
\hline $\mathfrak{K}(\tau)$ & delay power spectrum of the channel \\
\hline$\Re_{f a \dot{d e}}(\tau)$ & delay power spectrum of the diffused channel \\
\hline $\mathfrak{R}_{\mathrm{z}}\left(\Delta \mathrm{t} ;\left(\tau_{1}, \tau_{2}\right)\right)$ & autocorrelation function of $z(t, \tau)$ \\
\hline$\sigma$ & normalized delay parameter \\
\hline$\sigma_{\mathrm{e}}^{2}$ & variance of the additive error (complex Gaussian random variable) \\
\hline$\sigma_{n}$ & delay corresponding to the $n$th faded beam \\
\hline$\sigma_{N}^{2}$ & variance of the noise component \\
\hline$\theta_{n}$ & angle of arrival of the $n$th wave \\
\hline$\tau_{0}$ & delay corresponding to the direct/specular path \\
\hline$\tau_{\max }$ & maximum delay spread \\
\hline$\tau_{\min }$ & delay with respect to the first faded component \\
\hline$\tau_{n}(t)$ & $n$th path delay \\
\hline$\omega_{d}$ & maximum Doppler angular frequency \\
\hline
\end{tabular}




\section{Acknowledgment}

This thesis is a tribute to my parents, Shri Dinesh C. Jain and Smt. Trishla Jain for their infinite support and encouragement. I am equally grateful to my brother, Dr. Gaurav Dinesh and other near and dear ones for their well wishes and moral support throughout.

Of special mention is my thesis advisor, Dr. Cyril Leung, whose guidance and critical thinking helped me reach the goal. I would also like to thank my friends especially Cyril Iskander, Xinrong Wang and Farshid Agharebparast for providing with useful suggestions and help. Thanks also go to the members of my thesis examination committee for their time and efforts.

This work was supported by Natural Sciences and Engineering Research Council of Canada (NSERC) grant \#OGP0001731. 


\section{Chapter 1 Introduction}

Mankind has been on an unending quest to improve his communication ability. It began with the use of sign language and gradually developed into well defined speech. Communication at a distance was a challenge. The practice of sending handwritten messages/letters through messengers is a very old one and still in prevalence. However, the twentieth century has seen dramatic changes in the field of telecommunications: the telegraph, telephone, internet and cellular phones are but the most visible examples. For many people, the need to be able to communicate anytime, anywhere is satisfied using cellular systems. However the design of mobile communication systems presents challenging problems because of the random time-varying nature of the land mobile radio channels.

\subsection{Motivation}

One of the main problems with transmission over land mobile radio channels is the multipath fading phenomenon which results in amplitude and phase fluctuations of the received signal, at times causing significant dispersion leading to interference.

Various diversity schemes [1] have been proposed to combat fading. Most of the commonly used schemes involve multiple transmission and/or reception of the signal in space, time, frequency and polarization planes. Although these methods can lower the error rates, they often achieve this at the cost of different system parameters like the transmission rates, bandwidth and system complexity. Orthogonal Frequency Division Multiplexing (OFDM) [2] is a concept that works on a simple principle of inducing time diversity inherently in the system by lengthening the symbol period so that the system is more robust to impulse noise and channel fades. 
Since the coherence time of a channel is inversely proportional to the carrier frequency, the application of higher operating frequencies (in the $\mathrm{GHz}$ range), for fixed (or relatively less increase in the) transmission rates, would increase the fading rate in the channel resulting in the channel to be treated as time variant over a single symbol. Figure 1.1 illustrates the difference between a fast and a slow flat-fading channel response assuming a fixed symbol/pulse duration. It is important to realize that a system is referred to as slow or fast fading with respect to both, the channel parameter i.e. Doppler rate, $f_{d}$, and the symbol parameter i.e. pulse duration, $T_{p}$, which are together incorporated in the normalized Doppler rate, $f_{N}=f_{d} \cdot T_{p}$.
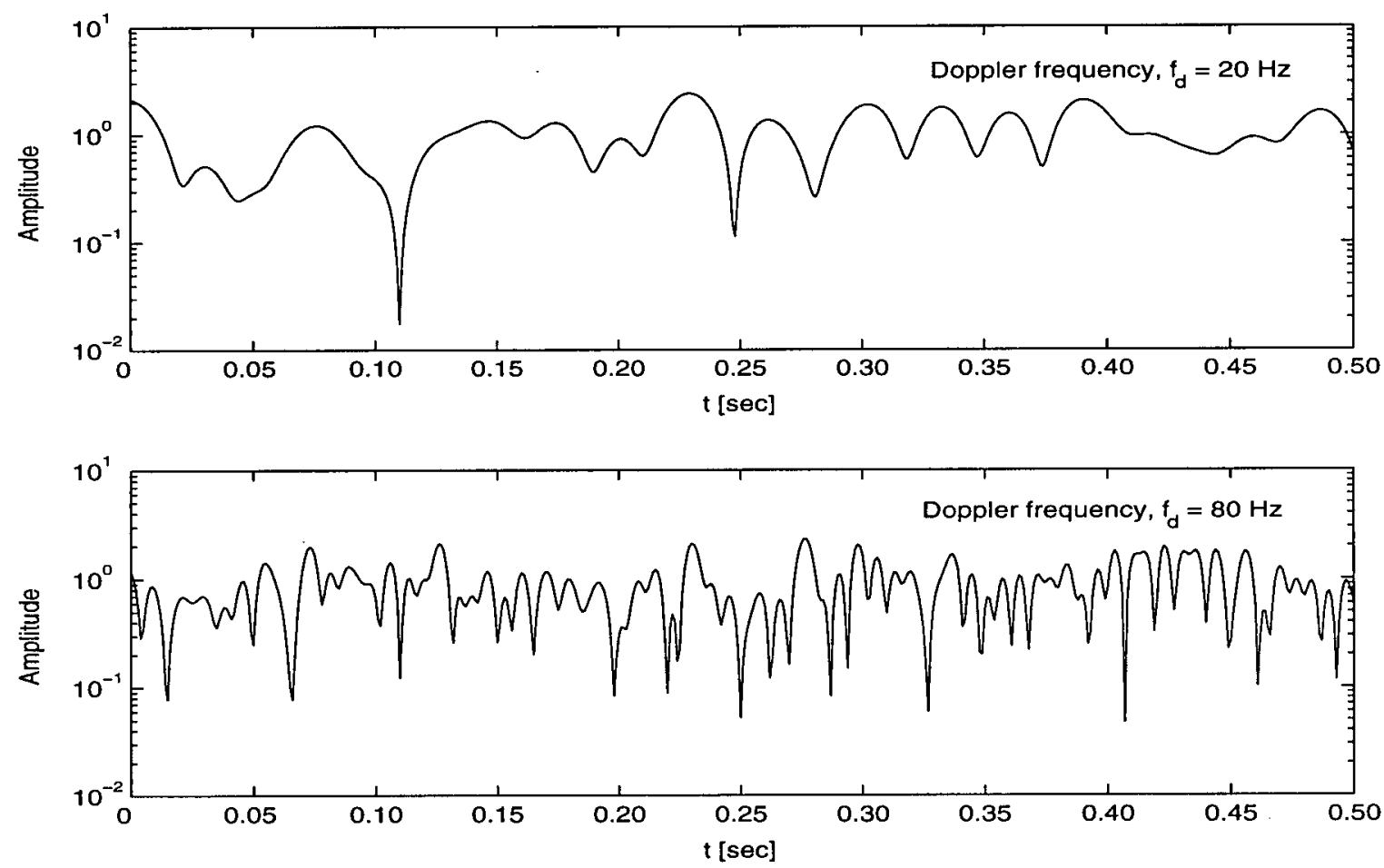

Figure 1.1 Received signal corresponding to slow and fast fading channels

In addition, as shown in [3], microcellular systems i.e. mobile radio systems configured with many small cells having low-powered base stations, often have Rician rather than Rayleigh envelope fading characteristics. The deployment of such systems in urban mobile radio channels 
and the use of multi-carrier modulation schemes like OFDM, provides the motivation for the study of the effect of frequency dispersion along with time dispersion in Rician fading channels.

\subsection{History of Related Work}

Communication over wireless channels has been studied since the early 1960's. In [4], the error probabilities for coherent and non-coherent multichannel (or multibranch) communication system involving Rician fading are obtained. The paper is among the pioneering works in obtaining the performance bounds for the coherent and non-coherent multichannel systems subjected to Rician and Rayleigh fading. It shows that the effectiveness of the multichannel reception is more pronounced in the case of channels with small Rician factors. However, it does not consider time dispersion within each of the channels.

Reference [5] discusses signal propagation through a Rayleigh fading channel without any explicit diversity (multichannel reception). The paper presents more general results than those in [4] by considering cases of a single-beam (flat-fading) and two-beam (frequency-selective fading) signal reception taking into account correlation between the beams. An extension of the above work appears in [6] which provides the performance analysis of a Rayleigh channel with continuous and discrete delay profiles for a space-diversity mobile radio receiver. This work compares the gain in bit error rate performance, obtained with space diversity over the gain obtained with frequency selectivity in the channel and the effectiveness of frequency selectivity, in lowering the error rate, in a single branch system over a multiple branch system.

The Rayleigh fading channel is a commonly used model when there is no line of sight (LOS) path between the transmitter and the receiver. When LOS path is present, a Rician fading model can be adopted. Matched filter bounds over a multipath Rician fading channel of discrete 
and continuous dispersion are derived in [7]. The paper emphasizes that the diversity gain achievable with intrinsic frequency diversity(IFD) (or frequency selectivity) diminishes with the increase in the Rician factor of the channel. It is worth noting that [4] and [7] refer to the diversity gain from different aspects. The former defines diversity gain with respect to multiple branch system when the diversity is induced in the system whereas the latter relates it to the IFD of the channel.

A more recent and useful contribution to the system presented in [6] appears in [8], [14] and [9] where the channel is no longer assumed to be time-invariant. The effect of fading rate in the Rayleigh fading channel is studied. In [8] a general algorithm for obtaining matched filter bounds for an uncoded BPSK modulation scheme is devised and the effect of pulse shape on receiver's performance is analyzed. Similar approach is applied in [9], although in the frequency domain and the assumption of uncorrelated scattering in the channel is made in deriving the results for a continuously dispersive channel. The exploitation of Doppler diversity (frequency dispersion) in fast fading channels is also discussed in [10].

\subsection{Thesis Contribution}

The research presented in the thesis involves deriving the optimum bit error rate performance of a system, comprising of a linear time-varying Rician fading channel, with uncoded BPSK modulation. In compliance with the previous results, [8] and [14], the probability of error is found to be significantly affected by the channel's fading rate and unlike in slow fading case, the shape of the transmitted pulse poses an issue. The system behavior as a function of different parameters like the Rician factor, normalized Doppler rate etc. for frequency flat and in addition, the delay spread and beam correlation for frequency selective channels is studied. Finally, bit 
error rate performance of a system employing a non-optimum receive filter is presented.

\subsection{Thesis Organization}

The research work, background information and conclusions are, in all, presented in five chapters, a brief description of which follows:

- Chapter 1: The chapter introduces the challenge undertaken in the present research and chronologically provides the background of the problem. It also specifies, in brief, the contributions of the thesis.

- Chapter 2: The objective is to provide a necessary and basic understanding of the fading phenomenon, the communication system, the wireless channel and other related topics.

- Chapter 3: This chapter forms the core of the thesis as it presents the solution to the problem stated in the introductory chapter. A detailed analysis of the matched filter bounds is presented.

- Chapter 4: All the analytical and simulation results are graphically presented in this chapter. It includes physical interpretation of the results and comparisons between some of the plots.

- Chapter 5: The conclusions derived from this research and some useful suggestions for future work in related field are presented in this chapter. 


\section{Chapter 2 The Wireless Channel}

\subsection{Introduction}

The effect of a fading wireless channel on a transmitted signal is both complex and interesting. The main purpose of this chapter is to provide a basic understanding of the fading phenomenon, its cause and effects; this is necessary background for the work presented in this thesis.

\subsection{Signal Propagation in a Wireless Channel}

In a wireless communication system, the transmitted signal often reaches its destination via multiple paths. The mechanisms that impact signal propagation can be classified into three types, namely reflection, diffraction and scattering [1], [11]:

- Reflection is caused by the radio wave striking and reflecting off a smooth surface with very large dimension compared to the signal wavelength, $\lambda$.

- Diffraction occurs due to the obstruction of the radio path between the transmitter and the receiver by a dense body with large dimension relative to $\lambda$ resulting in the formation of secondary waves behind the obstructing body.

- Scattering occurs when the signal impinges on either a large rough surface or any surface with dimension of the order of $\lambda$ or less, causing the reflected energy to scatter in all directions.

Fading is a cumulative effect of the above mechanisms.

\subsubsection{Land Mobile Fading}

The arrival of the transmitted signal at the receiver over multiple paths gives rise to the 
phenomenon of fading ${ }^{1}$, in which a signal undergoes random and time varying attenuation and delay.

It is useful to distinguish between two types of fading effects [1]:

Large-scale fading which represents average signal power attenuation or path loss due to motion over large areas and small-scale fading which refers to the changes in signal amplitude and phase owing to small changes in the location of the receiver with respect to the transmitter. Although both fading aspects are significant from different perspectives, the present work focuses on small-scale fading as encountered in land mobile fading channels.

It has been often observed that, due to the dense population of sky-scrapers and other tall structures within a city, there seldom exists a direct line of sight (LOS) path between a Base Station (BS) and a Mobile Station (MS) whereas in sub-urban areas this is usually not the case. Rather, there are generally some prominent structures which may act as fixed scatterers or signal reflectors. Thus, inspite of the lack of a LOS path, the MS may continue to receive a signal of a fixed, appreciable strength in addition to the randomly varying ${ }^{2}$ signals from other scatterers and reflectors which are in relative motion to the mobile.

Accordingly, the channel is modeled as Rician fading if it is characterized by the presence of LOS, fixed scatterers or signal reflectors in the medium or Rayleigh fading if the specular path (i.e. the non-fading component of the channel) is absent. The Rician fading channel is considered

1 When the multipath effect is caused by physical changes in the propagating medium for example variations in the density of ions in the ionospheric layers, it is referred as Scintillation. This is especially applicable in radio astronomy; however both terms, fading and scintillation, refer to a signal's random fluctuations and differ mainly in their causes of occurrence [11].

2 Here the random variations in the signal are being treated as variations with respect to different symbols or variations within a symbol of a finite duration. 
as a more general model in which the absence of the specular path results in the Rayleigh fading channel.

\subsubsection{Fading Channel}

In a wireless communication system, the received signal is often a random transformation of the transmitted one.

Two types of channel models have been proposed in [7]: continuously dispersive (CD) and discrete path (DP) channel models. A CD channel has a continuum of diffused components. For a transmitted signal, $s(t)$, the received signal can be written as [12]

$$
r_{c d}(t)=\int_{0}^{T_{M}} z_{c d}(t, \tau) \cdot s(t-\tau) d \tau
$$

where $T_{M}$ is the channel's delay spread and $z_{c d}(t, \tau)$ is the impulse response of the CD channel which is defined as the response of the channel at time $t$ to an impulse applied at time $t-\tau$.

On the other hand, we model the DP channel as consisting of a large number of discrete paths (multipath); each path $n$ has independent time varying attenuation, $\alpha_{n}(t)$, delay, $\tau_{n}(t)$, and phase, $\varphi_{n}(t)$, with respect to the transmitted signal, $s(t)$. The signal, $r_{d p}(t)$, at the channel output is

$$
r_{d p}(t)=\sum_{n} \alpha_{n}(t) \cdot e^{-j \varphi_{n}(t)} \cdot s\left(t-\tau_{n}(t)\right)
$$

The difference between the above two channel models can be clearly observed in Figure 2.1 which displays the response of a CD and DP channel to a short pulse (ideally an impulse). The 
received signal appears as a continuously time-varying signal or a train of pulses respectively.
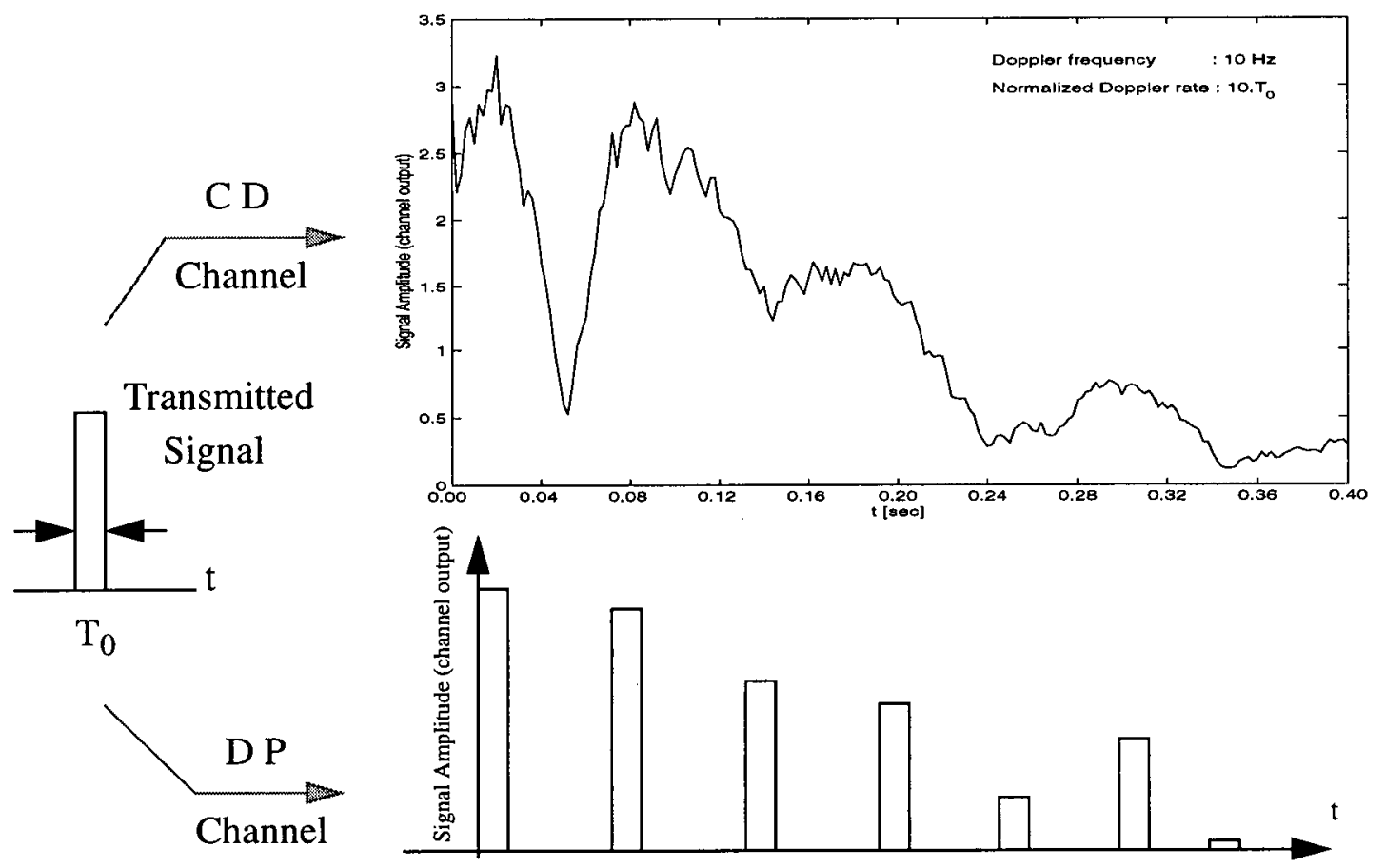

Figure 2.1 Continuously Dispersive and Discrete Path channel outputs

Small variations in the spatial separation between the transmitter and the receiver and/or random changes in the surroundings have two major manifestations:

- Time-spreading and

- Frequency-spreading of the signal

Time-spreading, commonly known as signal dispersion, is the elongation of the transmitted signal as it undergoes multipath propagation. It is caused by the fact that the different signal paths vary in length causing copies of the signal to reach the receiver at different times. As a result, not only is the signal spread in time but the signal components arriving at the receiver also differ in phase. Thus, a signal component with phase $\varphi_{2}$, transmitted at time $t_{2}$, may arrive in conjunction with another component of phase $\varphi_{1}$ transmitted at time $t_{1}$, resulting in constructive or 
destructive interference between the two. We can characterize signal dispersion in terms of Delay $\operatorname{Spread}\left(T_{M}\right)$. Viewed in the frequency domain, signal dispersion is expressed in terms of Coherence Bandwidth $\left(\Delta f_{c}\right)$ which implies that the signal components having frequency separation less than $\Delta f_{c}$ are treated alike by the channel. It can be shown [12] that $\Delta f_{c} \approx \frac{1}{T_{M}}$. Based on this, we can further classify fading as:

- Flat or frequency non-selective fading: When $\Delta f_{c}$ is large relative to the signal bandwidth, the channel is said to be flat. Viewed in the time domain, the channel ideally causes no delay spread in the transmitted signal, i.e. $T_{M}=0$.

- Frequency Selective fading: If the coherence bandwidth is small compared to the signal bandwidth, the channel is said to be frequency selective. The attendant delay spreading causes inter-symbol interference (ISI).

Frequency-spreading of the signal is a direct consequence of the spatial changes of the receiver relative to its surroundings, accounting for propagation path changes. As a result, the channel offers a time-variant response. This is characterized by the Coherence Time $\Delta t_{c}$, which is defined as the duration over which the channel's impulse response can be considered as timeinvariant. Coherence time is inversely proportional to the Doppler Spread, $\Delta t_{c} \propto \frac{1}{f_{d}}[12]$. The Doppler spread is a function of the MS velocity, $v$, and signal wavelength, $\lambda$, and is a measure of the spectral broadening of the signal

$$
f_{n}=f_{d} \cdot \cos \left(\theta_{n}\right)
$$

where $f_{n}$ is the Doppler shift [15] for the $n^{\text {th }}$ wave arriving at an angle $\theta_{n}$ with respect to the 
direction of motion of the MS and $f_{d}\left(f_{d}=\frac{v}{\lambda}\right)$ is the maximum Doppler frequency.

Depending on the channel's coherence time with respect to the signal duration, the Doppler spreading results in:

- Fast Fading: The channel exhibits a rapidly time-varying impulse response relative to the symbol duration and the signal experiences spectral broadening. Fast fading often causes the occurrence of frequent deep fades in the received signal.

- Slow Fading: When the channel's coherence time is long compared to the symbol duration, the channel is considered as slowly fading. Equivalently, the Doppler spread is small.

It is important to understand that the time-spreading and frequency-spreading are independent phenomenons. A wirreless channel may exhibit any combinations of Flat/Frequency Selective and Fast/Slow fading.

\subsection{Channel Model and its Statistical Characterization}

\subsubsection{The Linear Channel}

In terms of the complex lowpass equivalent form of a bandpass system [12], we can express the impulse response of the channel as $z(t, \tau)$, which represents the response of the channel at time $t$ to an impulse applied at time $t-\tau$. In this section an expression for $z(t, \tau)$ applicable for both flat and frequency selective fading channels is obtained. In doing so, we follow an approach similar to that of [12]. The general expression of the impulse response is given as

$$
z(t, \tau)=\sum_{n} \alpha_{n}(t) \cdot e^{-j 2 \pi f_{c} \tau_{n}(t)} \cdot \delta\left(\tau-\tau_{n}(t)\right)
$$

where $f_{c}$ is the carrier frequency of the bandpass signal; $\alpha_{n}(t)$ and $\tau_{n}(t)$ are the attenuation and 
delay of the $n^{\text {th }}$ ray at time $t$, respectively.

Equation (2.3.1) is a general expression from the point of view that it accounts for both Rician and Rayleigh fading channels and accommodates for all types of fading discussed in Section 2.2.2.

Since the specular path is a non-fading component of the channel, we can implement the impulse response of a Rician fading channel, $z_{\text {rice }}(t, \tau)$, by taking a constant attenuation, $\alpha_{0}$, and associated delay, $\tau_{0}$, for the first arriving ray

$$
z_{\text {rice }}(t, \tau)=\alpha_{0} \cdot e^{-j 2 \pi f_{c} \tau_{0}} \cdot \delta\left(\tau-\tau_{0}\right)+\sum_{n} \alpha_{n}(t) \cdot e^{-j 2 \pi f_{c} \tau_{n}(t)} \cdot \delta\left(\tau-\tau_{n}(t)\right)
$$

Figure 2.2 shows the block diagram representation of the response $r(t)$ of a fading channel $z(t, \tau)$, corrupted with noise $n(t)$, to an input $s(t)$.

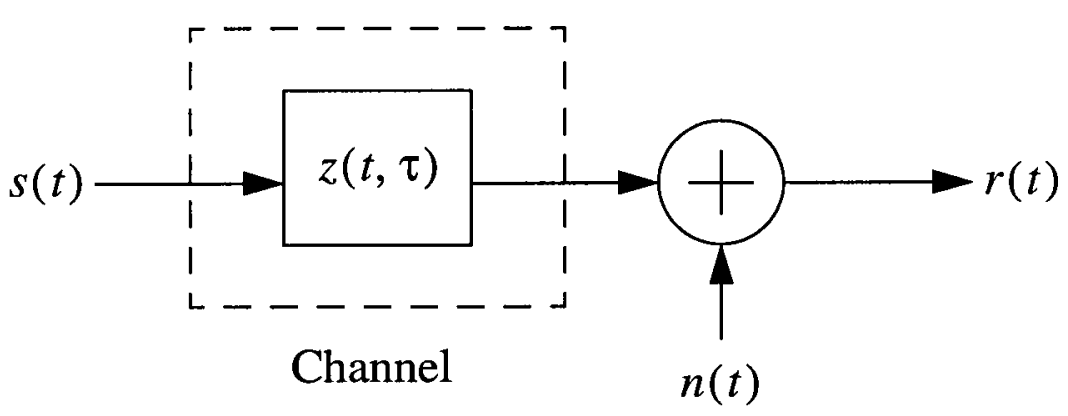

AWGN

Figure 2.2 Linear System model

The signal $r(t)$ can be written as

$$
r(t)=\int_{-\infty}^{\infty} z(t, \tau) s(t-\tau) d \tau+n(t)
$$

Substituting (2.3.1) in (2.3.3) and neglecting the noise term for the moment, the received signal, 
$r_{s}(t)$, is given as

$$
r_{s}(t)=\sum_{n} \alpha_{n}(t) \cdot e^{-j 2 \pi f_{c} \tau_{n}(t)} \cdot s\left(t-\tau_{n}(t)\right)
$$

The autocorrelation function of $z(t, \tau)$ is defined as [12]

$$
\Re_{z}\left(\Delta t ;\left(\tau_{1}, \tau_{2}\right)\right)=\frac{1}{2} \cdot E\left[z^{*}\left(t, \tau_{1}\right) \cdot z\left(t+\Delta t, \tau_{2}\right)\right]
$$

Assuming uncorrelated scattering, i.e. independence of attenuation and phase shifts associated with different path delays, and letting $\Delta t=0,(2.3 .5)$ can be re-written as

$$
\Re_{z}\left(0 ;\left(\tau_{1}, \tau_{2}\right)\right)=\Re\left(\tau_{1}\right) \cdot \delta\left(\tau_{1}-\tau_{2}\right)
$$

The function $\Re(\tau)$, known as the delay power spectrum of the channel, represents the average power output of the channel as a function of the time delay $\tau$. In urban environments, $\Re(\tau)$ can be approximated by the continuous one-sided exponential function [13], i.e.

$$
\Re(\tau)=\frac{1}{D_{r m s}} \cdot \exp \left(-\frac{\tau}{D_{r m s}}\right), \quad \tau \geq 0
$$

where $D_{r m s}$ is the rms delay spread. In some previous works [6], the class of two-sided continuous spectra is considered by assuming the Gaussian spectrum. However, for simplicity, we confine our analysis to one and two beam cases (Double-Spike spectrum (2.3.8)) which can easily be extended to a system with $\mathrm{N},(\mathrm{N}>2)$, beams by applying a similar procedure.

$$
\Re(\tau)=\frac{1}{2}(\delta(\tau)+\delta(\tau-\sigma))
$$

A snapshot of the impulse response with continuous one-sided exponential delay power spectrum is shown in Figure 2.3. The figure is plotted assuming $D_{r m s}=0.1 \mathrm{sec}$ and a Doppler 
frequency of $10 \mathrm{~Hz}$. One needs to be careful while comparing Figure 2.1, pertaining to the response of the fading channel to an impulse, with the three-dimensional plot of the impulse response, $|z(t, \tau)|$; since $z(t, \tau)$ is a function of two time variables, $t$ and $\tau$, whereas the response of the channel is a function of $t$ only. It is, however, possible to trace the channel's response to an impulse from the three-dimensional plot of it's impulse response.

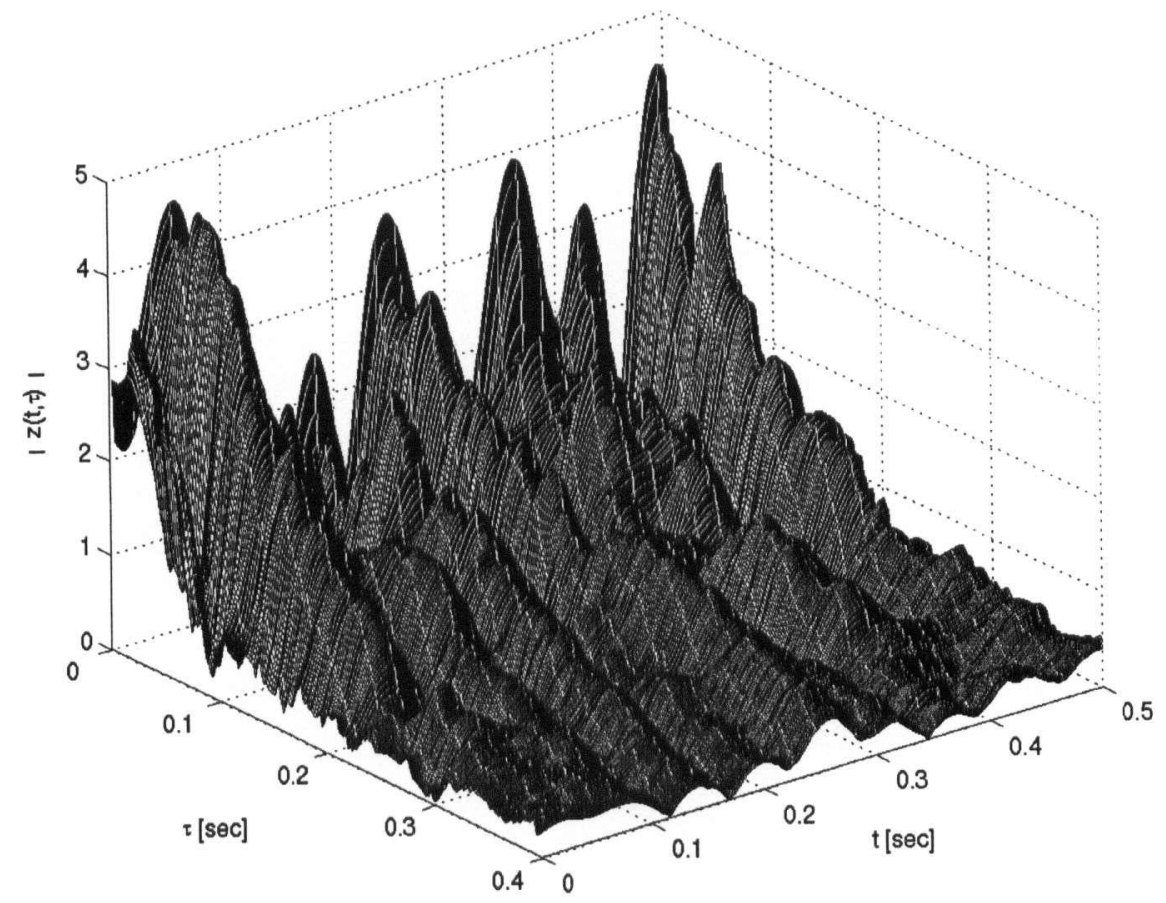

Figure 2.3 3-D view of the continuous-time impulse response, $|z(t, \tau)|$

It should be noted that the terms 'one beam' and 'two beam' models do not simply refer to the arrival of one and two rays at the mobile; rather each beam may be interpreted as a collection of several rays with varying path-delays closely distributed about the mean delays $\bar{\tau}_{1}$ and $\bar{\tau}_{2}$, respectively [14].

Thus, on splitting the second term in (2.3.2) into two parts (summation series) each reflecting the case of a single Rayleigh faded beam and denoting their respective indices by $n_{1}$ and $n_{2}$, 
with each ray in the group having an associated delay of $\tau_{n_{1}}(t)$ and $\tau_{n_{2}}(t)$, respectively, where

$$
\begin{aligned}
& \tau_{n_{1}}(t)=\bar{\tau}_{1}+\Delta \tau_{n_{1}}(t), \quad \Delta \tau_{n_{1}}(t) \ll T_{p} \\
& \tau_{n_{2}}(t)=\bar{\tau}_{2}+\Delta \tau_{n_{2}}(t), \quad \Delta \tau_{n_{2}}(t) \ll T_{p}
\end{aligned}
$$

$T_{p}$ is the transmitted signal block/pulse duration, we obtain

$$
z_{\text {rice }}(t, \tau)=\alpha_{0} \cdot e^{-j 2 \pi f_{c} \tau_{0}} \cdot \delta\left(\tau-\tau_{0}\right)+z_{1}(t, \tau)+z_{2}(t, \tau)
$$

where

$$
\begin{aligned}
& z_{1}(t, \tau)=e^{-j 2 \pi f_{c} \bar{\tau}_{1}} \sum_{n_{1}} \alpha_{n_{1}}(t) \cdot e^{-j 2 \pi f_{c} \Delta \tau_{n_{1}}(t)} \cdot \delta\left(\tau-\left(\bar{\tau}_{1}+\Delta \tau_{n_{1}}(t)\right)\right) \\
& z_{2}(t, \tau)=e^{-j 2 \pi f_{c} \bar{\tau}_{2}} \sum_{n_{2}} \alpha_{n_{2}}(t) \cdot e^{-j 2 \pi f_{c} \Delta \tau_{n_{2}}(t)} \cdot \delta\left(\tau-\left(\bar{\tau}_{2}+\Delta \tau_{n_{2}}(t)\right)\right) .
\end{aligned}
$$

An upper-bound on $\Delta \tau_{n}(t)$ can be obtained by realizing [14] that the greatest change in the path length occurs when the mobile is directly in line with the direction of arrival of the signal. Over the duration $T_{p}$, this would correspond to a change in path-length of $v \cdot T_{p}$ for a mobile moving at velocity $v$. Therefore the change in delay $\left(\Delta \tau_{n}(t)\right)_{\max }$ associated with the change in path-length would be $v \cdot T_{p} / c$, where $c$ is the speed of light. Since $\left(\Delta \tau_{n}(t)\right)_{\max }$ is much smaller than the pulse duration, we can drop the $\Delta \tau_{n}(t)$ factor from the delta functions in (2.3.11). However, the same cannot be neglected in the exponential terms due to the large value of $f_{c}$.

Assuming that each of the two beams consists of a large number of independent rays and 
the phases of the rays are uniformly distributed from 0 to $2 \pi$ [15], the Central Limit Theorem [16] can be applied to (2.3.11). Taking $\bar{\tau}_{1}=0$ and $\bar{\tau}_{2}=\sigma,(2.3 .10)$ simplifies to

$$
z_{\text {rice }}(t, \tau)=\alpha_{0} \cdot e^{-j 2 \pi f_{c} \tau_{0}} \cdot \delta\left(\tau-\tau_{0}\right)+z_{1}(t) \cdot \delta(\tau)+e^{-j 2 \pi f_{c} \sigma} z_{2}(t) \cdot \delta(\tau-\sigma)
$$

where $z_{1}(t)$ and $z_{2}(t)$ are complex Gaussian random processes accounting for the attenuation and phase of the two independent beams.

Since the direct/specular path is assumed to have the shortest path-length and the first Rayleigh faded component arrives with negligible delay, we have $\tau_{0}=0$. Normalizing the two random processes such that $E\left[\left|z_{1}(t)\right|^{2}\right]=E\left[\left|z_{2}(t)\right|^{2}\right]=1$ and explicitly specifying the strengths of the two Rayleigh faded beams as $\alpha_{1}$ and $\alpha_{2}$ respectively, we can re-write (2.3.12) as

$$
z_{\text {rice }}(t, \tau)=\alpha_{0} \cdot \delta(\tau)+\alpha_{1} z_{1}(t) \cdot \delta(\tau)+\alpha_{2} z_{2}(t) \cdot \delta(\tau-\sigma)
$$

Equation (2.3.13) is a general expression for the impulse response of a time-varying, frequency selective channel. The impulse response corresponding to a flat fading channel is

$$
z_{\text {rice }}(t, \tau)=\alpha_{0} \cdot \delta(\tau)+\alpha_{1} z_{1}(t) \cdot \delta(\tau)
$$

Figure 2.4 illustrates the impulse response corresponding to (2.3.13) assuming a unity gain channel with a Rician factor of 1 . The figure is plotted with a Doppler frequency of $20 \mathrm{~Hz}$ and a $\operatorname{delay}(\sigma)$ of $0.2 \mathrm{sec}$. 


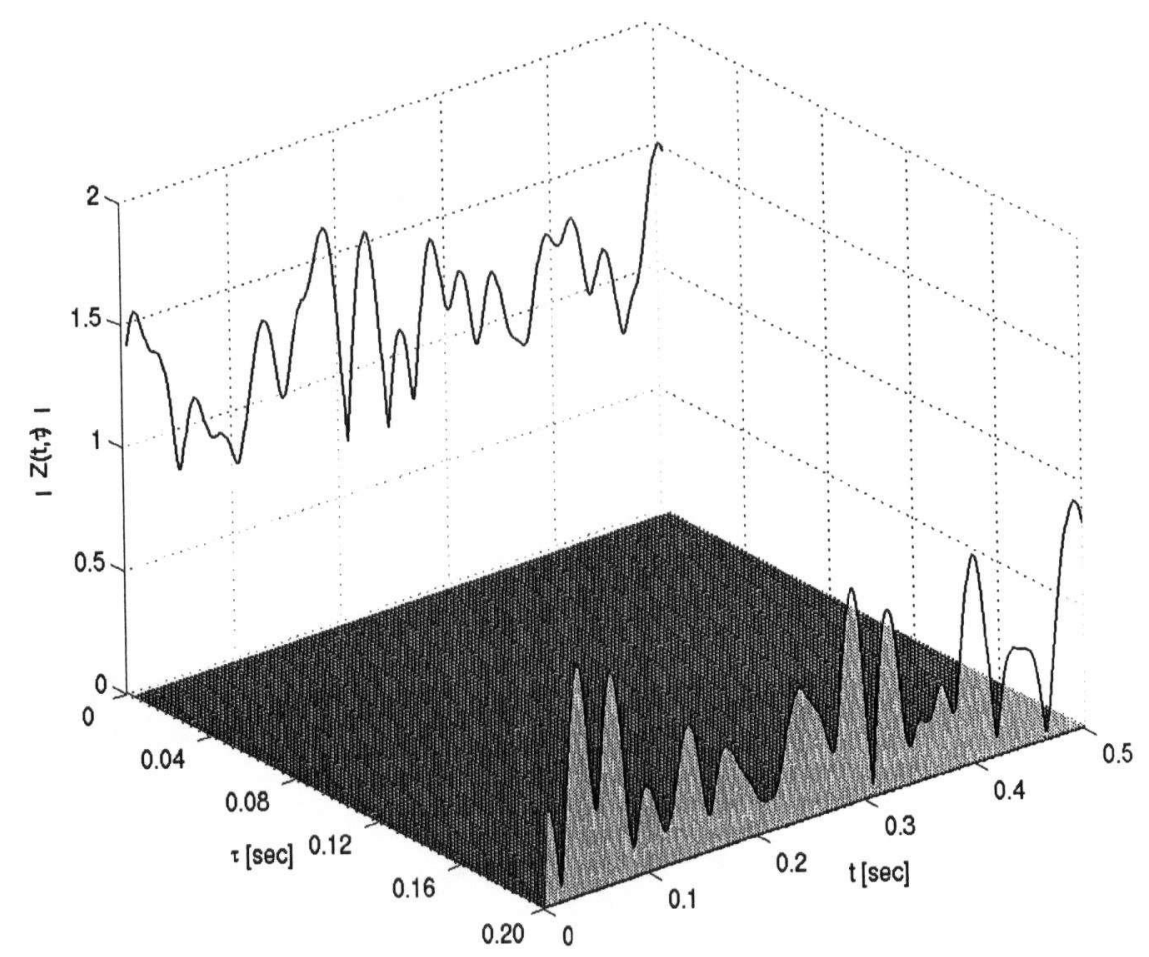

Figure $2.43-\mathrm{D}$ view of the discrete-time impulse response, $|z(t, \tau)|$

The Rician factor $K$ is defined as the power of the specular component relative to the power in the Rayleigh faded or diffused component and is often expressed in $\mathrm{dB}$. Thus

$$
K_{d B}=10 \cdot \log _{10}(K)=10 \cdot \log _{10}\left(\frac{\left(\alpha_{0}\right)^{2}}{\int \Re_{f a d e}(\tau) d \tau}\right)
$$

where $\mathfrak{R}_{\text {fade }}(\tau)$ is the average delay power spectrum of the fading component of the channel, $\left(\alpha_{0}\right)^{2}$ is the power of the specular path and the power of the diffused component is obtained by integrating the average delay power spectrum i.e. $\int \Re_{\text {fade }}(\tau) d \tau$.

\subsubsection{RF Spectrum and Autocorrelation of the Fading Process}

The present work is based on Clarke's model [17] which assumes a fixed transmitter with 
a vertically polarized antenna. The statistical characteristics of the random process $z_{i}(t)$ are based on isotropic scattering of the components/rays randomly arriving at the receiver at uniformly distributed angles and experiencing similar attenuation over small-scale distances.

Figure 2.5 depicts the spectral broadening of an unmodulated continuous wave (CW) carrier at frequency $f_{c}$ due to Doppler shift caused by the Rayleigh channel. Thus each of the rays arrives at the MS in its own frequency, offset from $f_{c}$ within $\pm f_{d}$. The corresponding power spectrum density $S(f)$ and autocorrelation function $\phi(t)$ of the Rayleigh fading process $z_{i}(t)$ for the case of a vertical whip antenna on the MS are given by [15]

$$
S(f)=\frac{3 \cdot b}{\omega_{d} \cdot\left(1-\left(\frac{f-f_{c}}{f_{d}}\right)^{2}\right)^{1 / 2}}
$$

where $b$ is the average power received by an isotropic antenna and $\omega_{d}=2 \pi f_{d}, f_{d}$ being the maximum Doppler frequency, and

$$
\phi(t)=1.5 \cdot b \cdot J_{0}\left(\omega_{d} \cdot t\right)
$$

where $J_{0}(x)$ is the zeroth order Bessel function of the first kind. 


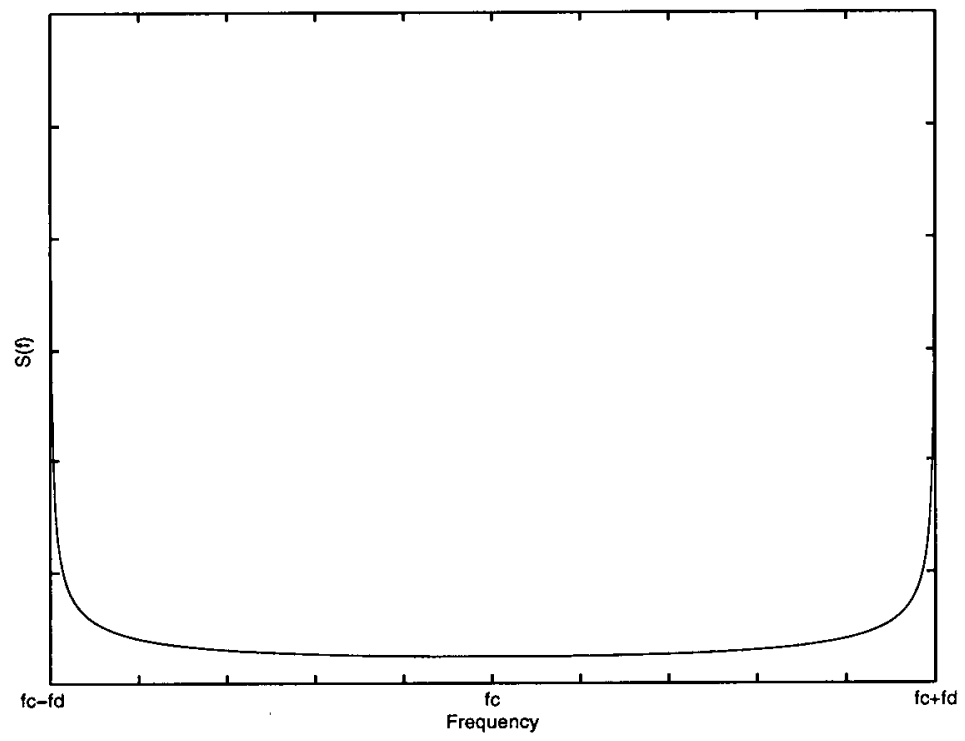

Figure 2.5 Power Spectrum of an unmodulated CW carrier 


\section{Chapter 3 Derivation of the MFB's}

\subsection{Introduction}

Matched Filter Bounds (MFB's) are useful to system designers in setting a goal for the practical realization of the system [6]. Assuming perfect equalization, the channel is considered free from Inter-symbol Interference (ISI). However the transmitted signal gets subjected to timevarying channel fades which, in most of the previous analyses, are assumed constant during a symbol duration. This chapter presents the MFB analysis for an uncoded BPSK system on a Rician fading channel with an arbitrary normalized Doppler rate. We assume an adaptive matched filter whose impulse response varies in accordance with the received (faded) signal.

By varying the Rician factor, the derived expressions can be used to obtain the bit error rate (BER) performance in strongly (Rayleigh) and weakly fading channels and compare their behavior under fast and slow fading conditions. Although developed for the cases of one and two beam (in addition to the non-fading component) channels, the analysis can be extended to include multiple $(>2)$ beams with arbitrary arrival times and inter-beam correlations.

We define a channel as slowly or fast fading with respect to the normalized Doppler rate $f_{N}=f_{d} \cdot T_{p}$ where $f_{d}$ is the Doppler frequency and $T_{p}$ is the symbol duration. In land mobile radio systems operating in the range of $900 \mathrm{MHz}$ to $1.8 \mathrm{GHz}, f_{d}$ typically ranges from 50 to 100 $\mathrm{Hz}$ for a vehicle speed of $60 \mathrm{~km} / \mathrm{hr}$. Therefore a system using a transmission rate of $10^{4}$ symbols/ sec would have a maximum normalized Doppler rate of 0.01 . This means that there are negligible variations in the channel characteristics over the duration $T_{p}$. We will refer to a system for which 
$f_{N}<0.01$ as slowly fading. For an OFDM system employing hundreds of tones, $T_{p}$ would be hundreds of times longer and in this case an analysis applicable to fast fading is required.

\subsection{System Model}

We assume BPSK modulation for simplicity, although the analysis presented in the chapter can be applied to any PAM scheme with minor modifications. Figure 3.1 shows the complex baseband representation of the communication system. A signal, $s(t)$, is sent by the modulator over the Rician channel. It is convenient to write

$$
s(t)= \pm A \cdot p(t), \quad A=\sqrt{E_{b}}
$$

where $p(t)$ is a unit energy pulse of duration $T_{p}$, so that $s(t)$ has energy $E_{b}$.

From Section 2.3.1, the complex baseband equivalent form of the impulse response of the time-varying channel is denoted by $z(t, \tau)$ which is defined as the response of the channel at time $t$ to an impulse applied at time $t-\tau$ [12]. The response $z(t, \tau)$ can be written as the sum of the impulse response of the direct path, $\alpha_{0} \cdot \delta(\tau)$, and the response of the diffused beam, $z_{\text {ray }}(t, \tau)$, i.e.

$$
z(t, \tau)=\alpha_{0} \cdot \delta(\tau)+z_{\text {ray }}(t, \tau)
$$

where $\alpha_{0}^{2}$ is the power of the direct/specular component. The real and imaginary parts of $z_{\text {ray }}(t, \tau)$ are independent and identically distributed (i.i.d.) zero mean Gaussian random processes.

The channel imposes a maximum delay spread of $\tau_{\max }$ on the transmitted signal $s(t)$. 


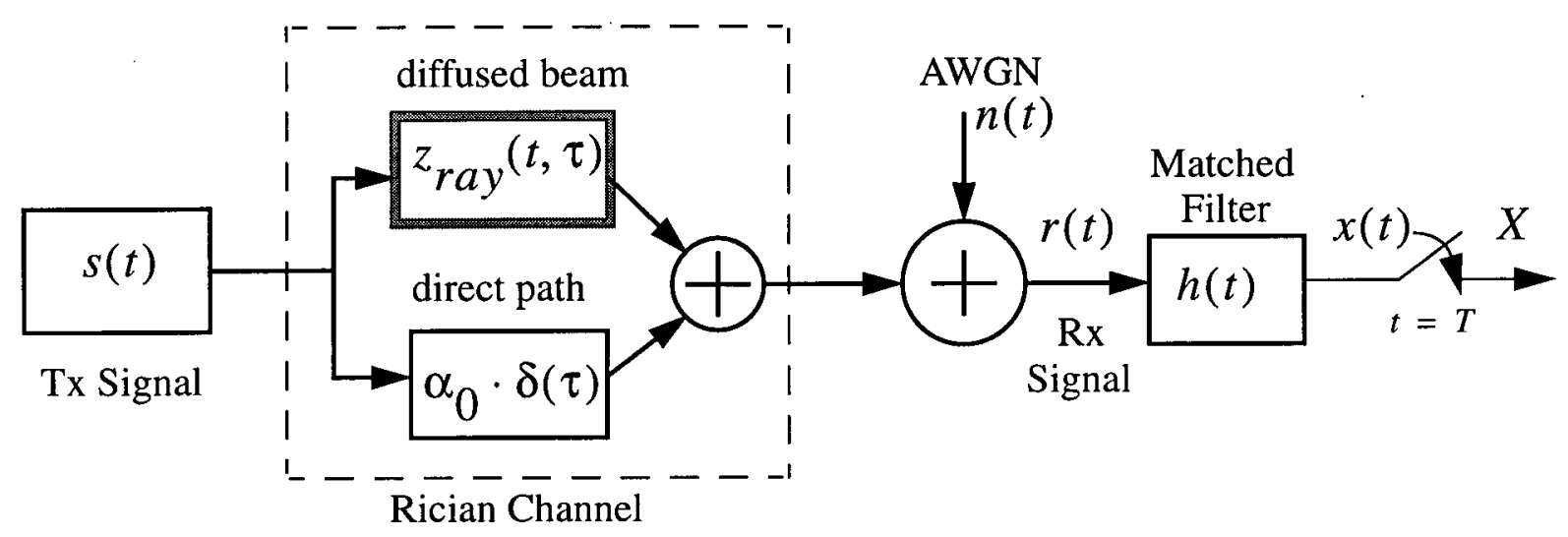

Figure 3.1 System Model

The received signal $r(t)$ is given by

$$
r(t)=\int_{0}^{\tau_{\max }} z(t, \tau) \cdot s(t-\tau) d \tau+n(t),
$$

where $n(t)$ is a sample function of the zero mean complex additive white Gaussian noise process with power spectral density $N_{0}$. The receiver's decision, as to which signal was transmitted, is based on the real component, $X_{r}$, of the output variable $X=x(T)$, where

$$
x(t)=\int_{0}^{T} h(t-\tau) \cdot r(\tau) d \tau, \quad T=T_{p}+\tau_{\max }
$$

\subsection{Error Performance Analysis}

The faded signal at the channel output is written as

$$
r_{f a d}(t)= \pm A \cdot s_{f a d}(t)
$$

where 


$$
\begin{aligned}
s_{f a d}(t) & =\int_{0}^{\tau_{\max }} z(t, \tau) \cdot p(t-\tau) d \tau \\
& =\alpha_{0} \cdot p(t)+\int_{\tau_{\min }}^{\tau_{\max }} z_{\text {ray }}(t, \tau) \cdot p(t-\tau) d \tau .
\end{aligned}
$$

In (3.3.2) we have assumed that the direct path has zero delay whereas the impulse response of the diffused beam, $z_{\text {ray }}(t, \tau)$, extends from $\tau_{\min }$ to $\tau_{\max }$.

It is well known that a matched filter (MF) is the optimal receiver for a signal transmitted over a non-fading AWGN channel [18]. Therefore, by having the impulse response, $h(t)$, of the receive filter matched to the complex signal, $s_{f a d}(t)$, we can obtain the optimum BER performance, i.e.

$$
h(t)=s_{f a d} *(T-t) .
$$

Thus the entire system in Figure 3.1 can be interpreted in the form shown in Figure 3.2. Using (3.3.3) in (3.2.4), we can write the filter output as

$$
x(t)=\int_{0}^{T} s_{f a d} *(T-t+\tau) r(\tau) d \tau
$$

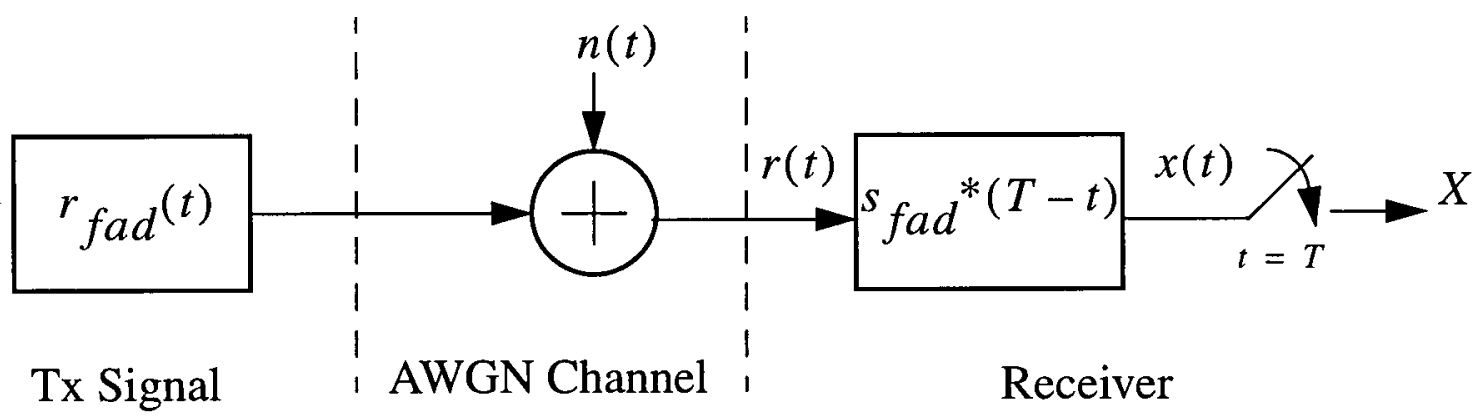

Figure 3.2 The Transformed System 
Therefore, the decision variable $X_{r}$ can be expressed as

$$
X_{r}= \pm A \cdot Y+N
$$

where the signal component, $Y$, is given by

$$
\begin{aligned}
Y & =\int_{0}^{T}\left|s_{f a d}(t)\right|^{2} d t \\
& =\int_{0}^{T}\left(\left|\alpha_{0} \cdot p(t)+\int_{\tau_{\min }}^{\tau_{\max }} z_{\text {ray }}(t, \tau) \cdot p(t-\tau) d \tau\right|^{2}\right) d t
\end{aligned}
$$

and the noise component, $N$, is given by

$$
\begin{aligned}
N & =\operatorname{Re}\left[\int_{0}^{T} s_{f a d}{ }^{*}(t) \cdot n(t) d t\right] \\
& =\operatorname{Re}\left[\int_{0}^{T}\left(\alpha_{0} \cdot p^{*}(t)+\int_{\tau_{\min }}^{\tau_{\max }} z_{\text {ray }}{ }^{*}(t, \tau) \cdot p^{*}(t-\tau) d \tau\right) \cdot n(t) d t\right] .
\end{aligned}
$$

In order to find the probability of error, we need to know the probability density function (pdf) of $X_{r}$. For convenience it is assumed that $s(t)=A \cdot p(t)$ is transmitted. Since the noise component, $N$, is Gaussian, the pdf of $X_{r}$ conditioned on the signal component, $Y$, is also Gaussian with mean $A \cdot Y$; it's variance can be obtained using Parseval's theorem [18] as

$$
\begin{aligned}
\sigma_{N}^{2} & =\frac{N_{0}}{2} \cdot \int\left|s_{f a d} *(T-t)\right|^{2} d t \\
& =\frac{N_{0}}{2} \cdot Y .
\end{aligned}
$$

Equation (3.3.8) shows that the noise variance, being a function of $Y$, is itself a random variable. 
The pdf of $X_{r}$ conditioned on $Y$ is given as

$$
f_{X_{r}}\left(x_{r} \mid y\right)=\frac{1}{\sqrt{2 \pi \sigma_{N}^{2}}} \exp \left[\frac{-\left(x_{r}-(A y)\right)^{2}}{2 \sigma_{N}^{2}}\right] \text {. }
$$

The conditional probability of error is the probability that $X_{r}$ is less than zero, i.e.

$$
P_{r}\left(X_{r}<0 \mid Y\right)=\int_{-\infty}^{0} f_{X_{r}}\left(x_{r} \mid y\right) d x_{r}
$$

The overall probability of error $P_{b}$ is obtained by averaging (3.3.10) over the pdf of $Y$

$$
P_{b}=\int_{-\infty}^{\infty} P_{r}\left(X_{r}<0 \mid Y\right) \cdot f_{Y}(y) d y .
$$

Using (3.3.9) and (3.3.10) and the fact that $Y \geq 0$ from (3.3.6), we can rewrite $P_{b}$ as

$$
P_{b}=\int_{0}^{\infty}\left(\int_{-\infty}^{0}\left(\frac{1}{\sqrt{2 \pi \sigma_{N}^{2}}} \exp \left[\frac{-\left(x_{r}-(A y)\right)^{2}}{2 \sigma_{N}^{2}}\right]\right) d x_{r}\right) \cdot f_{Y}(y) d y
$$

Equation (3.3.12) can be expressed in terms of the complementary error function, $\operatorname{erfc}(x)$, as

$$
\begin{aligned}
P_{b} & =\frac{1}{2} \cdot \int_{0}^{\infty} \operatorname{erfc}\left(\frac{A y}{\sqrt{2 \sigma_{N}^{2}}}\right) \cdot f_{Y}(y) d y \\
& =\frac{1}{2} \cdot \int_{0}^{\infty} \operatorname{erfc}\left(\sqrt{\frac{E_{b} \cdot y}{N_{0}}}\right) \cdot f_{Y}(y) d y .
\end{aligned}
$$

The term $E_{b} y / N_{0}$ can be interpreted as the received SNR where $E_{b} / N_{0}$ is the transmitted SNR. The BER can be calculated using (3.3.13) if the pdf $f_{Y}(y)$ is available. The determination of $f_{Y}(y)$ can be a challenging task. The following quote from [14] summarizes the problem, 
'The effects of fast fading, correlations and diversity are reflected solely in how they affect the pdf of $Y^{\prime}$.

\subsubsection{Deriving the pdf of $Y$}

Expanding (3.3.6), $Y$ can be written as

$$
Y=Y_{1}+Y_{2}+Y_{3}
$$

where

$$
\begin{gathered}
Y_{1}=\alpha_{0}^{2} \cdot \int_{0}^{T_{p}} p^{2}(t) d t=\alpha_{0}^{2} \\
Y_{2}=2 \alpha_{0} \int_{\tau_{\min }}^{T_{p}}\left(\operatorname{Re}\left[\int_{\tau_{\min }}^{\tau_{\max }} z_{\text {ray }}(t, \tau) \cdot p(t-\tau) d \tau\right] \cdot p(t)\right) d t \\
Y_{3}=\int_{\tau_{\min }}^{T}\left|\int_{\tau_{\min }}^{\tau_{\max }} z_{\text {ray }}(t, \tau) \cdot p(t-\tau) d \tau\right|^{2} d t \\
=\int_{\tau_{\min }}|f(t)|^{2} d t
\end{gathered}
$$

and $f(t)=\int_{\tau_{\min }}^{\tau_{\max }} z_{\text {ray }}(t, \tau) \cdot p(t-\tau) d \tau$. Following [14], we approximate (3.3.17) as a finite summation, i.e.

$$
Y_{3}=\Delta t \cdot \sum_{n=1}^{M}\left|f_{n}\right|^{2}
$$


where $M=\left\lfloor\frac{T_{p}+\tau_{\max }-\tau_{\min }}{\Delta t}\right\rfloor$ and $f_{n}=f\left(\left(n-\frac{1}{2}\right) \cdot \Delta t\right) ;$ the floor function $\lfloor x\rfloor$ is used to denote the largest integer $\leq x$. Since $f(t)$ is a zero mean complex Gaussian random process comprising of i.i.d. real and imaginary components, each of the $M$ terms, $\left|f_{n}\right|^{2}$, has a central chisquared degree 2 distribution. However (3.3.18) is a sum of correlated random variables. In order to express $Y_{3}$ as a sum of independent random variables, we use an orthogonal expansion (Karhunen-Lo'eve Expansion, [16], [19]), i.e.

$$
f_{n} \cdot \sqrt{\Delta t}=\sum_{k=1}^{M} U_{k} \cdot \sqrt{\lambda_{k}} \cdot \varphi_{n k}
$$

where $\left\{\varphi_{n k}\right\}=\left\{\varphi_{k}\left(\left(n-\frac{1}{2}\right) \cdot \Delta t\right)\right\}$ are orthonormal vectors (3.3.20) and $\left\{U_{k}\right\}$ are orthogonal complex zero-mean Gaussian random variables with unit variance as shown in (3.3.21)

$$
\begin{gathered}
\sum_{n=1}^{M} \varphi_{n l} \cdot \varphi_{n m}{ }^{*}=\delta_{l m}=\left\{\begin{array}{l}
1, l=m \\
0, l \neq m
\end{array}\right. \\
E\left(U_{l} \cdot U_{m}{ }^{*}\right)=\delta_{l m} .
\end{gathered}
$$

$\left\{\lambda_{k}\right\}$ and $\left\{\varphi_{k}(t)\right\}$ are recognized as the eigenvalues and eigen-functions [16] that satisfy the integral equation

$$
\int_{\tau_{\min }}^{T} R_{f}\left(t_{1}, t_{2}\right) \cdot \varphi_{k}\left(t_{2}\right) d t_{2}=\lambda_{k} \cdot \varphi_{k}\left(t_{1}\right)
$$

where $R_{f}\left(t_{1}, t_{2}\right)$ is the autocorrelation function of the process $f(t)$. The function 


$$
\begin{aligned}
R_{f}\left(t_{1}, t_{2}\right) & =E\left[f\left(t_{1}\right) \cdot f^{*}\left(t_{2}\right)\right] \\
& =E\left[\int_{\tau_{\min }}^{\tau_{\max } \tau_{\max }} \int_{\tau_{\max }} z_{\text {ray }}\left(t_{1}, \tau_{a}\right) z_{\text {ray }} *\left(t_{2}, \tau_{b}\right) p\left(t_{1}-\tau_{a}\right) p^{*}\left(t_{2}-\tau_{b}\right) d \tau_{a} d \tau_{b}\right]
\end{aligned}
$$

can be expressed as a Hermitian matrix $R_{f}$ whose $(l, m)$ th element is

$$
\begin{aligned}
R_{f(l, m)} & =E\left[f_{l} \cdot f_{m}{ }^{*}\right] \\
& =\int_{\tau_{\min }}^{\tau_{\max } \tau_{\max }} \int_{\boldsymbol{m}_{2}} \Re_{z}\left[(l-m) \Delta t ;\left(\tau_{a}, \tau_{b}\right)\right] p\left(l^{\prime} \Delta t-\tau_{a}\right) p^{*}\left(m^{\prime} \Delta t-\tau_{b}\right) d \tau_{a} d \tau_{b}
\end{aligned}
$$

where $l^{\prime}=l-\frac{1}{2}, m^{\prime}=m-\frac{1}{2}$ and $1 \leq l, m \leq M$. From (2.3.5), the autocorrelation of $z_{\text {ray }}(t, \tau)$ is given by

$$
\Re_{z}\left[t_{1}-t_{2} ;\left(\tau_{a}, \tau_{b}\right)\right]=E\left[z_{\text {ray }}\left(t_{1}, \tau_{a}\right) \cdot z_{\text {ray }}{ }^{*}\left(t_{2}, \tau_{b}\right)\right]
$$

Accommodating $\Delta t$ in (3.3.24) and using (3.3.19), we obtain

$$
R_{f} \cdot \Delta t=E\left[f \cdot f^{H}\right]=\varphi \cdot \Lambda \cdot \varphi^{H}
$$

where $f=\sqrt{\Delta t} \cdot\left[\begin{array}{lll}f_{1} & \ldots & f_{M}\end{array}\right]^{T}, \quad \varphi=\left[\begin{array}{lll}\varphi_{1} & \ldots & \varphi_{M}\end{array}\right] \quad$ with $\quad \varphi_{k}=\left[\begin{array}{lll}\varphi_{1 k} & \ldots & \varphi_{M k}\end{array}\right]^{T}$ and $\Lambda=\operatorname{diag}\left(\lambda_{1} \ldots \lambda_{M}\right)$. The eigenvectors are real and the eigenvalues are real and non-negative [20]. This also follows from [21] which shows that the eigenvalues of a positive definite matrix, $R_{f}$, are both real and positive.

$Y_{3}$ can now be expressed as a sum of uncorrelated random variables, $U_{1}, U_{2}, \ldots, U_{M}$. This follows from (3.3.18) and (3.3.19) from which we can write 


$$
\begin{aligned}
Y_{3} & =f^{H} \cdot f \\
& =\left[\sum_{k=1}^{M} U_{k} \sqrt{\lambda_{k}} \varphi_{1 k} \ldots \sum_{k=1}^{M} U_{k} \sqrt{\lambda_{k}} \varphi_{M k}\right] \cdot\left[\sum_{k=1}^{M} U_{k} \sqrt{\lambda_{k}} \varphi_{1 k} \cdots \sum_{k=1}^{M} U_{k} \sqrt{\lambda_{k}} \varphi_{M k}\right]^{T} \\
& =\sum_{k=1}^{M} \lambda_{k} \cdot\left|U_{k}\right|^{2}
\end{aligned}
$$

where the random variable, $U_{n}$, can be written as

$$
U_{n}=V_{n}+j W_{n}
$$

and $\left|U_{n}\right|^{2}=V_{n}^{2}+W_{n}^{2}$ has a central chi-squared degree 2 pdf. Also, from (3.3.19) and (3.3.21), the real component, $V_{n}$, and the imaginary component, $W_{n}$, of $U_{n}$ are i.i.d. zero-mean Gaussian random variables with variance $\sigma_{V_{n}}^{2}=\sigma_{W_{n}}^{2}=\frac{1}{2}$. The characteristic function of $Y_{3}$ is then easily obtained from the characteristic function of chi-squared degree 2 random variables as

$$
\Psi_{Y_{3}}(v)=\prod_{n=1}^{M} \frac{1}{\left(1-j \lambda_{n} v\right)}
$$

Equation (3.3.29) gives the characteristic function of the signal component $Y$ when the fading is Rayleigh.

Writing (3.3.16) in terms of $f(t)$ and using (3.3.19), we have

$$
\begin{aligned}
Y_{2} & =2 \alpha_{0} \int_{\tau_{\min }}^{T_{p}} \operatorname{Re}[f(t)] \cdot p(t) d t \\
& =2 \alpha_{0}\left(\sum_{i} \operatorname{Re}\left[f_{i^{\prime}}\right] \cdot p_{i} \cdot \Delta t\right) \\
& =2 \alpha_{0} \sum_{i=D}^{N}\left(\sum_{k=1}^{M} \operatorname{Re}\left[U_{k}\right] \cdot \sqrt{\lambda_{k}} \cdot \varphi_{i^{\prime} k}\right) \cdot p_{i} \cdot \sqrt{\Delta t}
\end{aligned}
$$


where $D=\left\{\begin{array}{c}\left\lfloor\frac{\tau_{\min }}{\Delta t}\right\rfloor, T_{p}>\tau_{\min }>0 \\ 1, \tau_{\min }=0\end{array}, N=\left\lfloor\frac{T_{p}}{\Delta t}\right\rfloor\right.$ and $i^{\prime}=i-D+1$

Interchanging the order of summations yields

$$
\begin{aligned}
Y_{2} & =2 \alpha_{0} \sqrt{\Delta t} \sum_{k=1}^{M} \operatorname{Re}\left[U_{k}\right] \cdot \sqrt{\lambda_{k}} \cdot\left(\sum_{i=D}^{N} \varphi_{i^{\prime} k} \cdot p_{i}\right) \\
& =\sum_{k=1}^{M} V_{k} \sqrt{\lambda_{k}} \cdot \beta_{k}
\end{aligned}
$$

where $\beta_{k}=2 \alpha_{0} \sqrt{\Delta t} \cdot \sum_{i=D}^{N} \varphi_{i^{\prime} k} \cdot p_{i}$ is a constant. Equation (3.3.31) suggests that $Y_{2}$ is a weighted sum of independent zero-mean real Gaussian random variables, $\left\{V_{k}\right\}$, with variance $\sigma_{V_{k}}^{2}$. Adding $Y_{2}, Y_{3}$ and simplifying, we obtain

$$
\begin{aligned}
Y_{3}+Y_{2} & =\left(\sum_{n=1}^{M} \lambda_{n} \cdot\left(V_{n}^{2}+W_{n}^{2}\right)\right)+\left(\sum_{n=1}^{M}\left(V_{n} \sqrt{\lambda_{n}}\right) \cdot \beta_{n}\right) \\
& =\sum_{n=1}^{M}\left(V_{n} \sqrt{\lambda_{n}}+\frac{\beta_{n}}{2}\right)^{2}-\sum_{n=1}^{M}\left(\frac{\beta_{n}}{2}\right)^{2}+\sum_{n=1}^{M}\left(\sqrt{\lambda_{n}} W_{n}\right)^{2}
\end{aligned}
$$

From (3.3.14) and (3.3.15), we have

$$
\begin{aligned}
Y & =\left(\alpha_{0}^{2}-\sum_{n=1}^{M}\left(\frac{\beta_{n}}{2}\right)^{2}\right)+\sum_{n=1}^{M}\left(V_{n}^{\prime}\right)^{2}+\sum_{n=1}^{M}\left(W_{n}{ }^{\prime}\right)^{2} \\
& =Y_{I}+Y_{I I}+Y_{I I I}
\end{aligned}
$$

where $V_{n}^{\prime}=V_{n} \sqrt{\lambda_{n}}+\frac{\beta_{n}}{2}$ and $W_{n}^{\prime}=W_{n} \sqrt{\lambda_{n}}$. In (3.3.33) $Y$ is represented as a sum of three independent terms. The characteristic function of $Y$ is therefore the product of the characteristic 
functions of each term. Realizing that the first term, $Y_{I}$, is a constant, its characteristic function is written as

$$
\Psi_{Y_{I}}(v)=\exp \left(j v\left(\alpha_{0}^{2}-\sum_{n=1}^{M}\left(\frac{\beta_{n}}{2}\right)^{2}\right)\right)
$$

The second term, $Y_{I I}$, has a non-central chi-squared distribution of degree 1 . Since $V_{n}^{\prime}$ has variance $\frac{\lambda_{n}}{2}$ and mean $\frac{\beta_{n}}{2}$, it's characteristic function is given by [12]

$$
\Psi_{Y_{I I}}(v)=\prod_{n=1}^{M} \frac{1}{\sqrt{\left(1-j \lambda_{n} v\right)}} \exp \left(\frac{j v \beta_{n}^{2}}{4\left(1-j \lambda_{n} v\right)}\right)
$$

The third term, $Y_{I I I}$, has a central chi-squared distribution of degree 1 with $W_{n}{ }^{\prime}$ having variance $\frac{\lambda_{n}}{2}$. Accordingly, we have from [12]

$$
\Psi_{Y_{I I}}(v)=\prod_{n=1}^{M} \frac{1}{\sqrt{\left(1-j \lambda_{n} v\right)}}
$$

The characteristic function of $Y$ is thus given by

$$
\begin{aligned}
\Psi_{Y}(v) & =\Psi_{Y_{l}}(v) \cdot \Psi_{Y_{I I}}(v) \cdot \Psi_{Y_{I I}}(v) \\
& =\exp \left(j v \alpha_{0}^{2}\right) \cdot \prod_{n=1}^{M} \frac{1}{\left(1-j \lambda_{n} v\right)} \exp \left(\frac{-v^{2} \beta_{n}^{2} \lambda_{n}}{4\left(1-j \lambda_{n} v\right)}\right)
\end{aligned}
$$

The corresponding pdf of $Y$ can be obtained numerically by taking the inverse Fourier transform of (3.3.37), i.e. 


$$
f_{Y}(y)=\frac{1}{2 \pi} \int_{-\infty}^{\infty} \Psi_{Y}(v) \exp (-j v y) d v
$$

\subsection{Flat Fading}

In flat fading, all the frequency components suffer similar attenuation and phase-shift by the channel and the delay power spectrum of the channel $\Re(\tau)$ is ideally an impulse. Therefore, for very-slow fading, we may write the channel's impulse response as

$$
z(t, \tau)=\alpha_{0} \delta(\tau)+Z \cdot \delta(\tau), \quad 0 \leq \alpha_{0} \leq 1
$$

where $Z$ is a complex Gaussian random variable; assuming that the channel has unity power gain, $E\left[|Z|^{2}\right]=1-\alpha_{0}^{2}$. However in the general case, when the attenuation may vary within the pulse duration, the channel's impulse response is given by (2.3.14)

$$
z(t, \tau)=\alpha_{0} \delta(\tau)+\alpha_{1} z(t) \delta(\tau)
$$

where $\alpha_{1}^{2}$ is the power of the diffused beam. The Rician factor of the channel is defined as

$$
K_{d B}=10 \cdot \log _{10}\left(\frac{\alpha_{0}^{2}}{\alpha_{1}^{2}}\right)
$$

$z(t)$ is a complex Gaussian random process with $E\left[|z(t)|^{2}\right]=1$. Thus from (3.2.2) $z_{\text {ray }}(t, \tau)=\alpha_{1} z(t) \delta(\tau)$ and the corresponding autocorrelation function is given by

$$
\begin{aligned}
\mathfrak{R}_{z}\left(t_{1}-t_{2} ;\left(\tau_{1}, \tau_{2}\right)\right) & =E\left[z\left(t_{1}\right) z^{*}\left(t_{2}\right)\right] \cdot \delta\left(t_{1}\right) \delta\left(t_{2}\right) \\
& =\phi_{z}\left(t_{1}-t_{2}\right) \cdot \delta\left(t_{1}\right) \delta\left(t_{2}\right)
\end{aligned}
$$

From (3.3.24) the $(l, m)$ th element of the corresponding Hermitian matrix $R_{f}$ is given by 


$$
R_{f(l, m)}=\int_{0}^{\tau_{\max } \tau_{\max }} \int_{0} \phi_{z}((l-m) \Delta t) \delta\left(\tau_{a}\right) \delta\left(\tau_{b}\right) p\left(l^{\prime} \Delta t-\tau_{a}\right) p^{*}\left(m^{\prime} \Delta t-\tau_{b}\right) d \tau_{a} d \tau_{b}
$$

Using the channel autocorrelation function for a mobile whip antenna with isotropic scattering [15]

$$
\phi_{z}(\tau)=J_{0}\left(\omega_{d} \cdot \tau\right)
$$

where $J_{0}(x)$ is a zeroth order Bessel function and $\omega_{d}=2 \pi f_{d}$ is the maximum Doppler angular frequency, (3.4.5) simplifies to

$$
R_{f(l, m)}=J_{0}\left(2 \pi f_{d}(l-m) \Delta t\right) p\left(l^{\prime} \Delta t\right) p^{*}\left(m^{\prime} \Delta t\right)
$$

The eigenvalues and eigenvectors corresponding to the matrix $R_{f} \Delta t$ can be easily obtained using mathematical tools [22]. Since $\tau_{\max }=\tau_{\min }=0$, from (3.3.30), $N=M$ and $i^{\prime}=i$ which gives

$$
\begin{aligned}
Y_{2} & =2 \alpha_{0} \alpha_{1} \sum_{i=1}^{M}\left(\sum_{k=1}^{M} V_{k} \sqrt{\lambda_{k}} \cdot \varphi_{i k}\right) p_{i} \sqrt{\Delta t} \\
& =\alpha_{1} \sum_{k=1}^{M}\left(V_{k} \sqrt{\lambda_{k}}\right) \cdot \beta_{k}
\end{aligned}
$$

and from (3.3.27) we have

$$
Y_{3}=\alpha_{1}^{2} \sum_{k=1}^{M} \lambda_{k}\left(V_{k}^{2}+W_{k}^{2}\right)
$$

Since the channel is assumed to be time-varying within the pulse duration, we expect the pulse shape to have a significant effect on the system performance as the effective channel averaging depends on the pulse shape. This can also be observed from the expression of the received 
SNR where the signal component $Y$ depends on the pulse $p(t)$. Therefore three pulse shapes are chosen

rect pulse:

$$
p_{\text {rect }}(t)= \begin{cases}1 / \sqrt{T_{0}}, & 0 \leq t \leq T_{0} \\ 0, & \text { otherwise }\end{cases}
$$

half sin pulse:

$$
p_{h s}(t)= \begin{cases}\sqrt{2 / T_{0}} \cdot \sin \left(\pi t / T_{0}\right), & 0 \leq t \leq T_{0} \\ 0, & \text { otherwise }\end{cases}
$$

root raised cosine pulse:

$$
p_{r s}(t)= \begin{cases}\sqrt{\frac{1}{2 T_{0}}\left(1+\cos \left(\frac{\pi\left(t-a T_{0}\right)}{a T_{0}}\right)\right)}, & 0 \leq t<a T_{0} \\ 1 / \sqrt{T_{0}}, & a T_{0} \leq t \leq T_{0} \\ \sqrt{\frac{1}{2 T_{0}}\left(1+\cos \left(\frac{\pi\left(t-T_{0}\right)}{a T_{0}}\right)\right)}, & T_{0}<t \leq T_{0}(1+a) \\ 0, & \text { otherwise }\end{cases}
$$

where $a$ is the roll-off factor of the pulse and $0 \leq a \leq 1$. The notation $T_{p}$, in general, refers to the symbol/pulse duration; henceforth $T_{p}=T_{0}(1+a)$ where $a=0$ for the rect pulse and the half sin pulse.

\subsubsection{Special Cases:}

\subsubsection{Very Slow Fading}

When the fading is very slow, $0<f_{N}<0.01$, using (3.4.1), the signal component $Y$ in 
(3.3.6) reduces to $Y=\left|\alpha_{0}+Z\right|^{2}$ which implies that $E[Y]=1.0$. This shows that the BER does not depend on the pulse shape and $P_{b}$ is calculated by averaging the error probability for a non-fading AWGN channel over the non-central chi-squared degree 2 pdf, i.e.

$$
P_{b}=\frac{1}{2} \cdot \int_{0}^{\infty} \operatorname{erfc}\left(\sqrt{\frac{E_{b} \cdot y}{N_{0}}}\right) \cdot \frac{1}{\left(1-\alpha_{0}^{2}\right)} \exp \left(-\frac{\alpha_{0}^{2}+y}{1-\alpha_{0}^{2}}\right) I_{0}\left(\frac{2 \sqrt{y} \alpha_{0}}{1-\alpha_{0}^{2}}\right) d y .
$$

Equation (3.4.13) is simplified in [4] and $P_{b}$ is expressed in terms of the Marcum's $Q$-function

$$
P_{b}=Q(v, w)-\frac{1}{2}\left(1+\sqrt{\frac{\varepsilon}{1+\varepsilon}}\right) \exp \left(-\frac{v^{2}+w^{2}}{2}\right) I_{0}(v w)
$$

where $\varepsilon=E\left[|Z|^{2}\right] \cdot E_{b} / N_{0}$ and

$$
\begin{aligned}
& v=\sqrt{\frac{K[1+2 \varepsilon-2 \sqrt{\varepsilon(1+\varepsilon)}]}{2(1+\varepsilon)}} \\
& w=\sqrt{\frac{K[1+2 \varepsilon+2 \sqrt{\varepsilon(1+\varepsilon)}]}{2(1+\varepsilon)}}
\end{aligned}
$$

$K$ is the Rician factor, $K=\alpha_{0}^{2} / E\left[|Z|^{2}\right], I_{\beta}(x)$ is the $\beta$ th order modified Bessel function of the first kind and the Marcum's $Q$-function is defined as [12]

$$
Q(v, w)=\exp \left(-\frac{v^{2}+w^{2}}{2}\right) \sum_{n=0}^{\infty}\left(\frac{v}{w}\right)^{n} I_{n}(v w), \quad w>v>0
$$

\subsubsection{Very Fast Fading}

It has been shown in Appendix B that in the extreme condition when the normalized Doppler rate increases without limit, i.e. $f_{N} \rightarrow \infty$, the variance of the signal component $Y$ approaches zero. Since the mean, $E[Y]=1.0$, this implies that the pdf, 
$\lim _{f_{N} \rightarrow \infty} f_{Y}(y)=\delta(y-1)$. Applying this in (3.3.13), we obtain

$$
\begin{aligned}
\lim _{f_{N} \rightarrow \infty} P_{b} & =\frac{1}{2} \cdot \int_{0}^{\infty} \operatorname{erfc}\left(\sqrt{\frac{E_{b} \cdot y}{N_{0}}}\right) \cdot \delta(y-1) d y \\
& =\frac{1}{2} \cdot \operatorname{erfc}\left(\sqrt{\frac{E_{b}}{N_{0}}}\right)
\end{aligned}
$$

which gives the BER for a non-fading AWGN channel. Thus using an extremely long signaling pulse relative to the channel's coherence time or having a very high Doppler frequency has essentially made the fading channel look like a non-fading one [14].

\subsubsection{Rician Factor}

Equation (3.3.13) gives the general expression of the BER for a Rician fading channel. Varying the Rician factor, $K$, corresponds to varying the power of the non-fading component with respect to that of the fading component. We consider two extreme cases corresponding to a Rayleigh channel $(K=0)$ and a non-fading channel $(K \rightarrow \infty)$.

When $K=0, \alpha_{0}=0$ and the direct/specular path is absent. Accordingly, the signal component $Y$ in (3.3.14) equals $Y_{3}$. Using (3.4.2) with $\alpha_{1}=E\left[|z(t)|^{2}\right]=1$ and $\alpha_{0}=0$, we have the following results from [8]

$$
P_{b}= \begin{cases}\frac{1}{2}\left(1-\sqrt{\frac{E_{b} / N_{0}}{E_{b} / N_{0}+1}}\right), & 0<f_{N}<0.01 \\ \frac{1}{2}\left(1-\sum_{n=1}^{L} \prod_{\substack{m=1 \\ m \neq n}}^{L} \frac{\lambda_{n}}{\lambda_{n}-\lambda_{m}} \cdot \sqrt{\frac{\lambda_{n}\left(E_{b} / N_{0}\right)}{\lambda_{n}\left(E_{b} / N_{0}\right)+1}}\right), & \text { otherwise }\end{cases}
$$


where $L$ is the number of non-zero eigenvalues of the matrix $R_{f} \Delta t$. On the other extreme when $K \rightarrow \infty$, the channel's impulse response (3.4.2) reduces to that of a non-fading AWGN channel and the BER is given by

$$
\lim _{K \rightarrow \infty} P_{b}=\frac{1}{2} \operatorname{erfc}\left(\sqrt{\alpha_{0}^{2} E_{b} / N_{0}}\right)
$$

\subsection{Frequency-Selective Fading}

When there is a significant amount of delay spread relative to the pulse duration, the channel is said to be frequency selective. This implies that the channel is selective in it's response to the signal at different frequencies and the fading at different frequencies is not necessarily correlated. We present the general cases of two and three beam signal reception when the direct (non-faded) and diffused versions of the transmitted pulse arrive at different time instants.

\subsubsection{Two-Beam Case}

In our two-beam case, the channel is modeled with the following impulse response

$$
z(t, \tau)=\alpha_{0} \delta(\tau)+\alpha_{1} z_{1}(t) \delta\left(\tau-\sigma_{1}\right)
$$

Assuming that the non-faded beam has the shortest path length, the first and the second terms correspond to the non-delayed (direct component) and delayed (faded component) responses of the channel respectively. Considering a unity gain channel, $\alpha_{1}=\sqrt{1-\alpha_{0}^{2}}$ and $E\left[\left|z_{1}(t)\right|^{2}\right]=1$. By varying the delay parameter $\sigma_{1}$, we can study the influence of signal dispersion on the system performance.

The signal component $Y$ for the present case is written as 


$$
Y=\alpha_{0}^{2}+2 \alpha_{0} \alpha_{1} \int_{\sigma_{1}}^{T_{p}} \operatorname{Re}\left[z_{1}(t) p\left(t-\sigma_{1}\right)\right] p(t) d t+\alpha_{1}^{2} \cdot \int_{\sigma_{1}}^{T}\left|z_{1}(t) p\left(t-\sigma_{1}\right)\right|^{2} d t
$$

where $T=T_{p}+\sigma_{1}$. Using (3.3.30) and (3.3.31), the second term in (3.5.2) can be written as

$$
\begin{aligned}
Y_{2} & =2 \alpha_{0} \alpha_{1} \sqrt{\Delta t} \sum_{i=D}^{N}\left(\sum_{k=1}^{M} V_{k} \sqrt{\lambda_{k}} \cdot \varphi_{i^{\prime} k}\right) p_{i} \\
& =\alpha_{1} \sum_{k=1}^{M} V_{k} \sqrt{\lambda_{k}} \cdot \beta_{k}
\end{aligned}
$$

where

$$
D=\left\{\begin{array}{l}
\left\lfloor\frac{\sigma_{1}}{\Delta t}\right\rfloor, \sigma_{1}>0 \\
1, \sigma_{1}=0
\end{array}\right.
$$

and $N=\left\lfloor\frac{T_{p}}{\Delta t}\right\rfloor, i^{\prime}=i-D+1 .\left\{\lambda_{k}\right\}$ are the eigenvalues of the matrix $R_{f} \cdot \Delta t ;$ the $(l, m)$ th element of matrix $R_{f}$ is defined in (3.4.7).

When $\sigma_{1} \geq T_{p}, Y_{2}=0$ which gives

$$
\begin{aligned}
Y & =\alpha_{0}^{2}+\alpha_{1}^{2} \cdot \int_{\sigma_{1}}^{T}\left|z_{1}(t) p\left(t-\sigma_{1}\right)\right|^{2} d t \\
& =\alpha_{0}^{2}+\alpha_{1}^{2} \cdot Y_{\text {ray }}
\end{aligned}
$$

where $Y_{\text {ray }}=\int_{\sigma_{1}}^{T}\left|z_{1}(t) p\left(t-\sigma_{1}\right)\right|^{2} d t$ is the received signal component from the diffused beam. 
The pdf of $Y$, obtained from the pdf of $Y_{\text {ray }}$ [14], is given as

$$
f_{Y}(y)= \begin{cases}\frac{1}{\alpha_{1}^{2}} \sum_{n=1}^{L} \frac{B_{n}}{\lambda_{n}} \exp \left(-\frac{\left(y-\alpha_{0}^{2}\right)}{\alpha_{1}^{2} \cdot \lambda_{n}}\right), & y \geq \alpha_{0}^{2} \\ 0, & \text { otherwise }\end{cases}
$$

where the constants $B_{n}=\left[\left(1-j \lambda_{n} v\right) \cdot \prod_{i=1}^{L} \frac{1}{\left(1-j \lambda_{i} v\right)}\right]_{v=\frac{1}{j \lambda_{n}}}$ and $L$ is the number of non-zero eigenvalues. The expression for the BER is thus given by

$$
P_{b}=\frac{1}{2 \alpha_{1}^{2}} \cdot \int_{\alpha_{0}^{2}}^{\infty} \operatorname{erfc}\left(\sqrt{\frac{E_{b} \cdot y}{N_{0}}}\right) \cdot \sum_{n=1}^{L} \frac{B_{n}}{\lambda_{n}} \exp \left(-\frac{\left(y-\alpha_{0}^{2}\right)}{\alpha_{1}^{2} \cdot \lambda_{n}}\right) d y .
$$

Performing integration by parts on (3.5.7) we get

$$
P_{b}=\frac{1}{2 \alpha_{1}^{2}} \sum_{n=1}^{L} \frac{B_{n}}{\lambda_{n}} \exp \left(\frac{\alpha_{0}^{2}}{\alpha_{1}^{2} \lambda_{n}}\right)\left(-\left.e r f c\left(\sqrt{\frac{E_{b} y}{N_{0}}}\right) e^{-\frac{y}{\alpha_{1}^{2} \cdot \lambda_{n}} \alpha_{1}^{2}} \lambda_{n}\right|_{\alpha_{0}^{2}} ^{\infty}-\alpha_{1}^{2} \lambda_{n} \sqrt{\frac{E_{b}}{N_{0} \pi}} \int_{\alpha_{0}^{2}}^{\infty} e^{\left[-y q_{n}\right]} \cdot y^{-1 / 2} d y\right)
$$

and using (A.10)

$$
P_{b}=\frac{1}{2} \sum_{n=1}^{L} B_{n}\left[\operatorname{erfc}\left(\sqrt{\frac{E_{b} \alpha_{0}^{2}}{N_{0}}}\right)-\sqrt{\frac{E_{b}}{N_{0} q_{n}}} \operatorname{erfc}\left(\sqrt{\alpha_{0}^{2} q_{n}}\right) \exp \left(\frac{\alpha_{0}^{2}}{\alpha_{1}^{2} \lambda_{n}}\right)\right]
$$

where $q_{n}=\frac{E_{b}}{N_{0}}+\frac{1}{\alpha_{1}^{2} \lambda_{n}}$ are constants.

For a very-slow fading case (with any degree of overlapping between the direct and the diffused beams), (3.5.1) and (3.5.2) are given as

$$
z(t, \tau)=\alpha_{0} \delta(\tau)+Z_{1} \delta\left(\tau-\sigma_{1}\right)
$$




$$
\begin{aligned}
Y & =\alpha_{0}^{2}+2 \alpha_{0} k \cdot Z_{r 1}+\left(Z_{r 1}^{2}+Z_{i 1}^{2}\right) \\
& =\alpha_{0}^{2}\left(1-k^{2}\right)+\left(Z_{r 1}+\alpha_{0} k\right)^{2}+Z_{i 1}^{2}
\end{aligned}
$$

where $Z_{1}=Z_{r 1}+j Z_{i 1}, E\left[\left|Z_{1}\right|^{2}\right]=E\left[Z_{r 1}^{2}+Z_{i 1}^{2}\right]=1-\alpha_{0}^{2} ; Z_{r 1}$ and $Z_{i 1}$ are i.i.d. zero mean Gaussian random variables and $k=\int_{\sigma_{1}}^{T_{p}} p(t) p\left(t-\sigma_{1}\right) d t$ is a constant. By employing simple transformations to the non-central chi-squared degree 2 distribution, the pdf of $Y$ is written as

$$
f_{Y}(y)=\frac{1}{1-\alpha_{0}^{2}} \exp \left(-\frac{2 \alpha_{0}^{2} k^{2}-\alpha_{0}^{2}+y}{1-\alpha_{0}^{2}}\right) I_{0}\left(\frac{\sqrt{y-\alpha_{0}^{2}\left(1-k^{2}\right)} \cdot 2 \alpha_{0} k}{1-\alpha_{0}^{2}}\right)
$$

When the two beams are non-overlapping, $\sigma_{1} \geq T_{p}$ and $k=0$. Consequently (3.3.13) simplifies to

$$
P_{b}=\frac{1}{2\left(1-\alpha_{0}^{2}\right)} \cdot \int_{\alpha_{0}^{2}}^{\infty} \operatorname{erfc}\left(\sqrt{\frac{E_{b} \cdot y}{N_{0}}}\right) \cdot \exp \left(-\frac{y-\alpha_{0}^{2}}{1-\alpha_{0}^{2}}\right) d y
$$

Applying a procedure similar to the one used in obtaining (3.5.9), the BER is given by

$$
P_{b}=\frac{1}{2}\left[\operatorname{erfc}\left(\sqrt{\frac{E_{b} \alpha_{0}^{2}}{N_{0}}}\right)-\exp \left(\frac{\alpha_{0}^{2}}{1-\alpha_{0}^{2}}\right) \sqrt{\frac{E_{b} / N_{0}}{\frac{E_{b}}{N_{0}}+\frac{1}{1-\alpha_{0}^{2}}}} \cdot \operatorname{erfc}\left(\sqrt{\alpha_{0}^{2}\left(\frac{E_{b}}{N_{0}}+\frac{1}{1-\alpha_{0}^{2}}\right)}\right)\right]
$$

The above expression can also be directly obtained from (3.5.9) by realizing that the matrix $R_{f} \cdot \Delta t$ has only a single non-zero eigenvalue, $\lambda_{1}=1.0$, when the fading is very slow. 


\subsubsection{Three-Beam Case}

In the three-beam channel model, the transmitted signal arrives following three propagation paths of which the first (non-delayed) one is the direct component and the other two arriving at some delays, $\sigma_{1}$ and $\sigma_{2}$, are the diffused components. Accordingly the impulse response is given by

$$
z(t, \tau)=\alpha_{0} \delta(\tau)+\alpha_{1} z_{1}(t) \delta\left(\tau-\sigma_{1}\right)+\alpha_{2} z_{2}(t) \delta\left(\tau-\sigma_{2}\right), \quad \sigma_{2} \geq \sigma_{1}
$$

Comparing (3.2.2) with (3.5.15), $z_{\text {ray }}(t, \tau)=\alpha_{1} z_{1}(t) \delta\left(\tau-\sigma_{1}\right)+\alpha_{2} z_{2}(t) \delta\left(\tau-\sigma_{2}\right)$. Assuming a unity gain channel and $E\left[\left|z_{1}(t)\right|^{2}\right]=E\left[\left|z_{2}(t)\right|^{2}\right]=1$, we have

$$
\alpha_{0}^{2}+\alpha_{1}^{2}+\alpha_{2}^{2}=1
$$

The signal component corresponding to (3.5.15) is

$$
Y=\alpha_{0}^{2}+2 \alpha_{0} \int_{\sigma_{1}}^{T_{p}} \operatorname{Re}[f(t)] p(t) d t+\int_{\sigma_{1}}^{T}|f(t)|^{2} d t
$$

where, from Section 3.3.1, $f(t)=\alpha_{1} z_{1}(t) p\left(t-\sigma_{1}\right)+\alpha_{2} z_{2}(t) p\left(t-\sigma_{2}\right)$. Considering the general case where the two processes $z_{1}(t)$ and $z_{2}(t)$ have a normalized cross-correlation coefficient $\rho$, which is given as

$$
\rho=\frac{\phi_{z_{1} z_{2}}(\tau)}{\sqrt{\phi_{z_{1}}(\tau)} \cdot \sqrt{\phi_{z_{2}}(\tau)}}, \quad|\rho| \leq 1
$$

where $\phi_{z_{1} z_{2}}(\tau)$ is the cross-correlation function and $\phi_{z_{i}}(\tau), i=1,2$, is the autocorrelation function of the two processes. Assuming both the fading processes have identical statistical characteristics, we can write 


$$
\phi_{z_{1}}(\tau)=\phi_{z_{2}}(\tau)=J_{0}\left(\omega_{d} \cdot \tau\right)
$$

The autocorrelation function of $z_{\text {ray }}(t, \tau)$ is given as

$$
\begin{aligned}
\Re_{z}\left(t_{1}-t_{2} ;\left(\tau_{1}, \tau_{2}\right)\right)= & \alpha_{1}^{2} \cdot \phi_{z_{1}}\left(t_{1}-t_{2}\right) \delta\left(\tau_{1}-\sigma_{1}\right) \delta\left(\tau_{2}-\sigma_{1}\right) \\
& +\alpha_{2}^{2} \cdot \phi_{z_{2}}\left(t_{1}-t_{2}\right) \delta\left(\tau_{1}-\sigma_{2}\right) \delta\left(\tau_{2}-\sigma_{2}\right) \\
& +\alpha_{1} \alpha_{2} \rho \phi_{z_{1}}\left(t_{1}-t_{2}\right) \delta\left(\tau_{1}-\sigma_{1}\right) \delta\left(\tau_{2}-\sigma_{2}\right) \\
& +\alpha_{2} \alpha_{1} \rho^{*} \phi_{z_{1}}\left(t_{1}-t_{2}\right) \delta\left(\tau_{1}-\sigma_{2}\right) \delta\left(\tau_{2}-\sigma_{1}\right)
\end{aligned}
$$

which results in the following elements of matrix $R_{f}$

$$
\begin{aligned}
R_{f(l, m)} & =J_{0}\left(\omega_{d}(l-m) \Delta t\right)\left[\alpha_{1}^{2} p\left(l^{\prime} \Delta t-\sigma_{1}\right) p^{*}\left(m^{\prime} \Delta t-\sigma_{1}\right)+\alpha_{2}^{2} p\left(l^{\prime} \Delta t-\sigma_{2}\right) p^{*}\left(m^{\prime} \Delta t-\sigma_{2}\right)\right. \\
& \left.+\alpha_{1} \alpha_{2} \rho p\left(l^{\prime} \Delta t-\sigma_{1}\right) p^{*}\left(m^{\prime} \Delta t-\sigma_{2}\right)+\alpha_{1} \alpha_{2} \rho^{*} p\left(l^{\prime} \Delta t-\sigma_{2}\right) p^{*}\left(m^{\prime} \Delta t-\sigma_{1}\right)\right]
\end{aligned}
$$

Using (3.3.31)

$$
\begin{aligned}
Y_{2} & =2 \alpha_{0} \sqrt{\Delta t} \sum_{i=D}^{N}\left(\sum_{k=1}^{M} V_{k} \sqrt{\lambda_{k}} \cdot \varphi_{i^{\prime} k}\right) p_{i} \\
& =\sum_{k=1}^{M} V_{k} \sqrt{\lambda_{k}} \beta_{k}
\end{aligned}
$$

When the first two beams arrive simultaneously i.e. $\sigma_{1}=0$, the three-beam case may be interpreted as a two-beam signal reception of which the first one is Rician faded and the second one is Rayleigh faded. Under these circumstances, for the special case of slow fading Rician channel when the fading in the two diffused components is uncorrelated, $\alpha_{1}=\alpha_{2}$ and $\sigma_{2} \geq T_{p}$, the BER is given as (Appendix A)

$$
P_{b}=\frac{1}{2} \sum_{n=0}^{\infty} C_{n}\left[1-\sqrt{\frac{\lambda-1}{\lambda}}-\sum_{k=1}^{n+1} 2^{-2 k+1}\left(\begin{array}{c}
2 k-1 \\
k
\end{array}\right) \sqrt{\frac{\lambda-1}{\lambda}} \cdot\left(\frac{1}{\lambda}\right)^{k}\right]
$$


where $\lambda=1+\frac{E_{b}\left(1-\alpha_{0}^{2}\right)}{2 N_{0}}$ and the coefficients $C_{n}$ are defined in (A.13).

In another case when $\sigma_{1} \geq T_{0}(1+a)$, i.e. there is no overlap between the direct and the diffused beams, (3.5.17) reduces to

$$
\begin{aligned}
Y & =\alpha_{0}^{2}+\int_{\sigma_{1}}^{T}|f(t)|^{2} d t \\
& =\alpha_{0}^{2}+Y_{\text {ray }}
\end{aligned}
$$

where $Y_{\text {ray }}=\int_{\sigma_{1}}^{T}|f(t)|^{2} d t$. Comparing (3.5.24) with (3.3.14), we have $Y_{1}=\alpha_{0}^{2}, Y_{2}=0$ and $Y_{3}=Y_{\text {ray }}$. The pdf of $Y$ derived from the pdf of $Y_{\text {ray }}$ [14] is given as

$$
f_{Y}(y)=\left\{\begin{array}{l}
\sum_{l=1}^{L_{d}} \sum_{m=1}^{r_{l}} \frac{B_{l m}}{(m-1) ! \lambda_{l}^{m}} \cdot\left(y-\alpha_{0}^{2}\right)^{m-1} \exp \left(-\frac{\left(y-\alpha_{0}^{2}\right)}{\lambda_{l}}\right), y \geq \alpha_{0}^{2} \\
0, \text { otherwise }
\end{array}\right.
$$

where $L_{d}$ is the number of distinct and non-zero eigenvalues, $\left\{\lambda_{l}\right\}$, and $r_{l}$ is the order of $\lambda_{l}$. The constants $B_{l m}$ are given by

$$
B_{l m}=\frac{1}{\left(r_{l}-m\right) !\left(-j \lambda_{l}\right)^{r_{l}-m}}\left[\frac{d^{r_{l}-m}}{d v^{r_{l}-m}}(1-j \lambda, v)^{r_{l}} \Psi_{Y_{\text {ray }}}(v)\right] v=\frac{1}{j \lambda_{l}}
$$

where $\Psi_{Y_{r a y}}(v)=\prod_{i=1}^{M} \frac{1}{\left(1-j \lambda_{i} v\right)}$ is the characteristic function of $Y_{r a y}$.

Combining (3.5.25) with (3.3.13) yields 


$$
P_{b}=\frac{1}{2} \sum_{l=1}^{L_{d}} \sum_{m=1}^{r_{l}} \frac{B_{l m}}{(m-1) ! \lambda_{l}^{m}} \int_{\alpha_{0}^{2}}^{\infty} \operatorname{erfc}\left(\sqrt{\frac{E_{b} y}{N_{0}}}\right) \cdot\left(y-\alpha_{0}^{2}\right)^{m-1} \exp \left(-\frac{\left(y-\alpha_{0}^{2}\right)}{\lambda_{l}}\right) d y
$$

Evaluating the above integral (Appendix C) results in the following expression

$$
\begin{aligned}
& P_{b}=\frac{1}{2}-\frac{1}{2} \sum_{l=1}^{L_{d}} \sum_{m=1}^{r_{l}} \frac{B_{l m}}{(m-1) ! \lambda_{l}^{m}}\left[\operatorname{erf}\left(\sqrt{\frac{E_{b} \alpha_{0}^{2}}{N_{0}}}\right)(m-1) ! \lambda_{l}^{m}\right. \\
&+\sqrt{\frac{E_{b}}{N_{0} \pi}} \sum_{n=0}^{m-1} \frac{(m-1) ! \lambda_{l}^{n+1}}{(\mu-1) !} \exp \left[\left(\frac{1}{\lambda_{l}}-\frac{C_{l}}{2}\right) \alpha_{0}^{2}\right] C_{l}^{-\frac{\left(\mu+\frac{1}{2}\right)}{2}} \alpha_{0}^{\left(\mu-\frac{3}{2}\right)} \Gamma(\mu) W_{\frac{1}{2}-\mu} \frac{1}{2}-\mu \\
& 2
\end{aligned}
$$

where $\mu=m-n$ and constants $C_{l}=\frac{E_{b}}{N_{0}}+\frac{1}{\lambda_{l}} \cdot \Gamma(z)$ is the Gamma function and $W_{\lambda, v}(z)$ is known as the Whittaker function [23]. $r_{l}=2, \forall l$ applies when the two faded beams are independent and non-overlapping. However when $r_{l}=1, \forall l,(3.5 .28)$ simplifies to

$$
P_{b}=\frac{1}{2}-\frac{1}{2} \sum_{l=1}^{L_{d}} B_{l 1}\left[\operatorname{erf}\left(\sqrt{\frac{E_{b} \alpha_{0}^{2}}{N_{0}}}\right)+\sqrt{\frac{E_{b}}{N_{0} \pi}} \exp \left(\frac{\alpha_{0}^{2}}{\lambda_{l}}\right) \sqrt{\frac{\pi}{C_{l}}} \operatorname{erfc}\left(\sqrt{C_{l} \alpha_{0}^{2}}\right)\right]
$$

where $\operatorname{erf}(x)$ and $\operatorname{erfc}(x)$ are the error functions and complementary error functions respectively. 


\section{Chapter 4 Numerical Results}

\subsection{Introduction}

The expressions obtained from the analysis in Chapter 3 are used to obtain BER curves for different channel conditions. The results for flat fading Rician and frequency selective fading Rician channels are presented in Section 4.2 and Section 4.3 respectively. In the figures, the BER plotted against $E_{b} / N_{0}$ assuming a unity gain channel. Unless otherwise stated, a rect pulse shape is assumed.

In order to verify the results from our analysis, simulations using MATLAB ${ }^{\circledR}$ [22] were performed for all the curves presented. The Rayleigh fading simulator used in the simulation is based on Clarke's model, [17], and uses a modified version, [24], of the algorithm in [25]. In all cases, the simulation results were found to agree closely with the analytical curves. The $99 \%$ confidence intervals for the simulation results are within $\pm 5 \%$ of the corresponding mean values. Because of the close agreement, the simulation results are generally not plotted.

The results in Section 4.2 and Section 4.3 assume that the receiver has perfect knowledge of the channel. In Section 4.4, the effect of channel estimation errors is studied.

\subsection{Flat Rician Fading}

Figure 4.1 illustrates the BER, $P_{b}$, curves for different normalized Doppler rates, $f_{N}$, with a Rician factor of $0 \mathrm{~dB}$. The limiting case of very fast fading $\left(f_{N} \rightarrow \infty\right)$ which coincides with the non-fading AWGN channel case (Section 3.4.1.2) is also shown. The pair of dots marked at intervals of $2 \mathrm{~dB}$ depict the confidence intervals. It is observed that the system performance 
improves substantially with the fading rate.

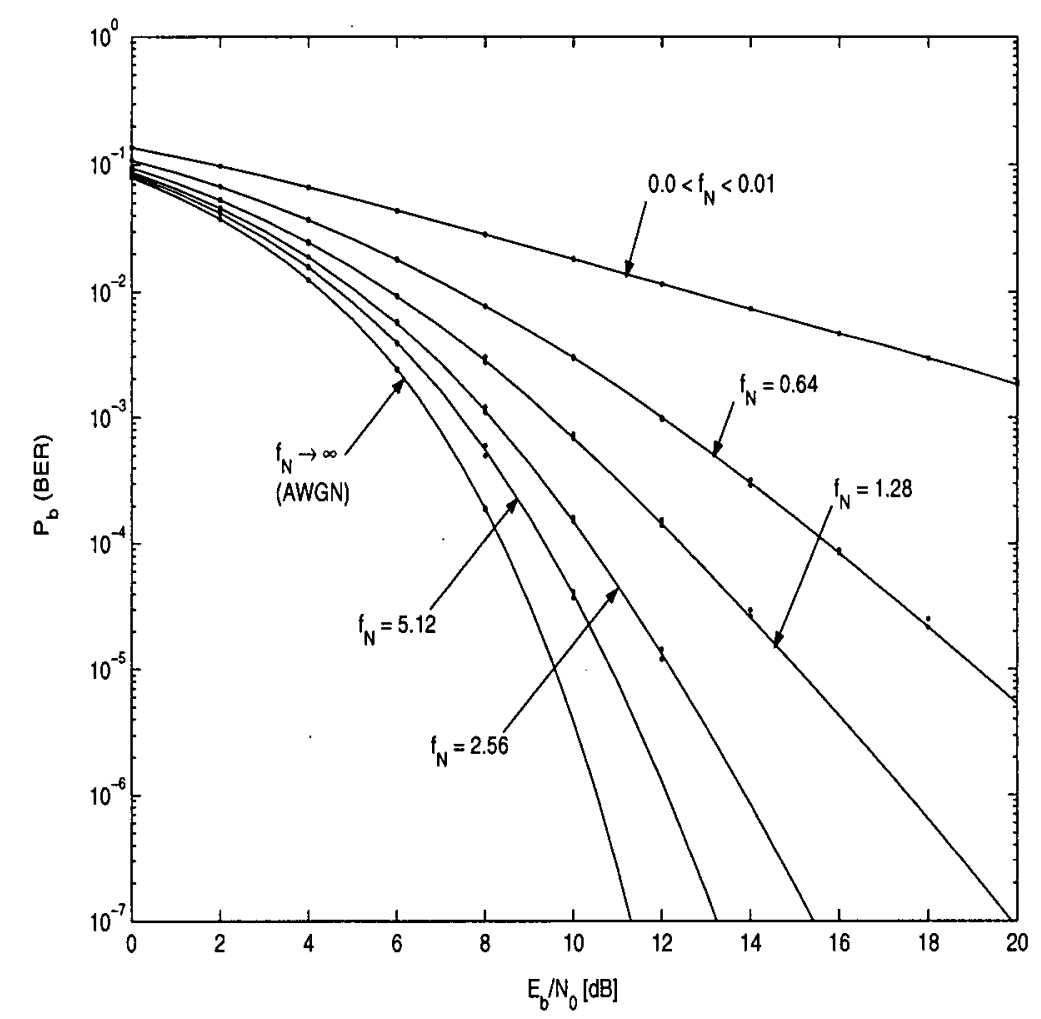

Figure 4.1 BER as a function of $f_{N}$ for the Rician channel, $K_{d B}=0 \mathrm{~dB}$

The case $0.0<f_{N}<0.01$, corresponding to very-slow fading, has the poorest performance and the curve shifts towards the non-fading case as $f_{N}$ increases. The improvement in performance with $f_{N}$ is more noticeable as we move from the very-slow fading to $f_{N}=0.64$ and it tends to diminish with further increase in $f_{N}$.

A comparison of the performance for the Rayleigh $\left(K_{d B} \rightarrow-\infty \mathrm{dB}\right)$, Rician $\left(K_{d B}=0 \mathrm{~dB}\right)$ and non-fading $\left(K_{d B} \rightarrow \infty \mathrm{dB}\right)$ channels as a function of $f_{N}$ is shown in Figure 4.2. As expected, the Rician channel has a lower BER than the Rayleigh channel. 


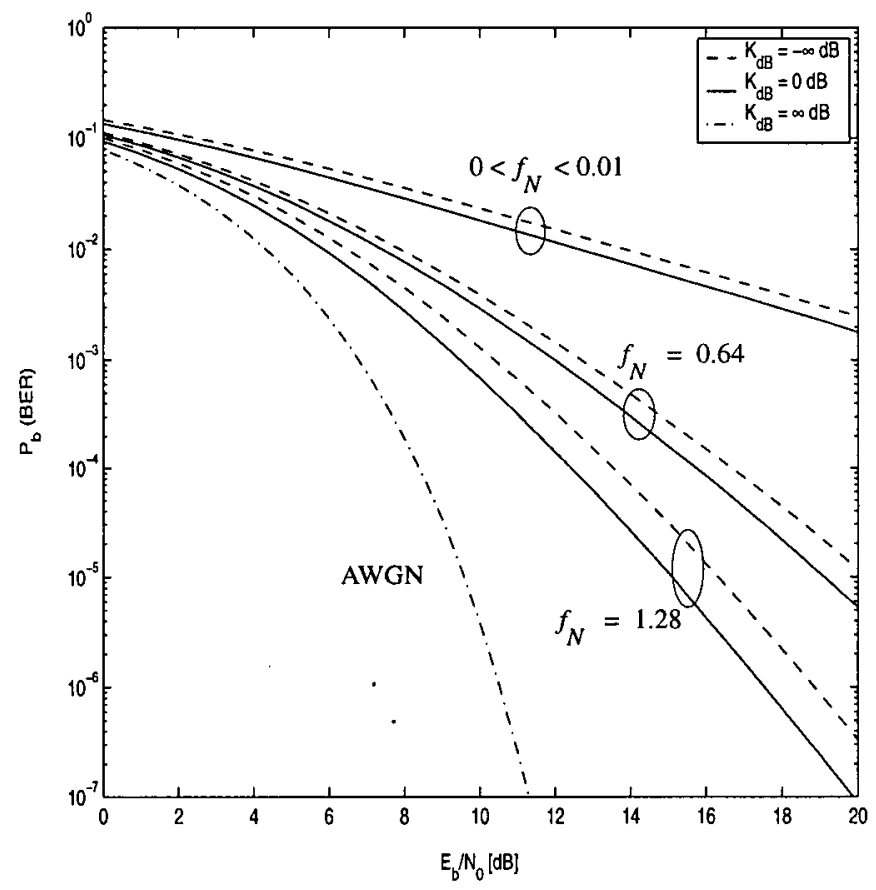

Figure 4.2 BER as a function of the Rician factor and $f_{N}$

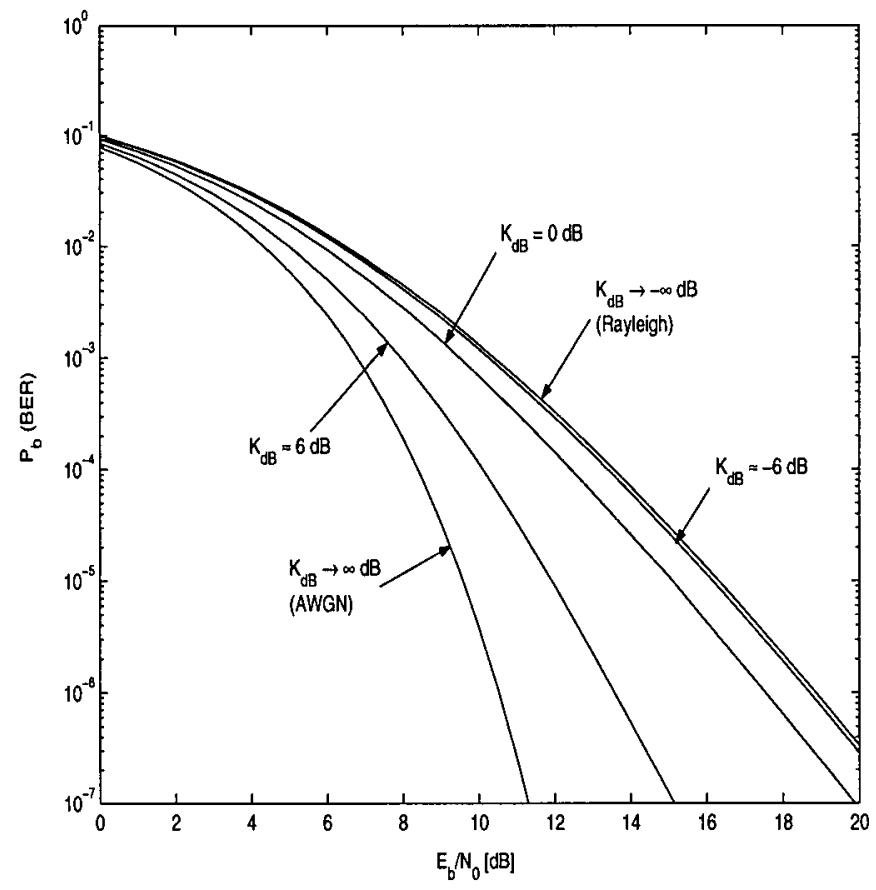

Figure 4.3 Effect of the Rician factor for $f_{N}=1.28$ 
In Figure 4.2 , for the values of $f_{N}$ chosen, the gap between the Rayleigh and Rician curves, for a given $E_{b} / N_{0}$, tends to increase with $f_{N}$. However, the two curves converge to the AWGN curve as $f_{N} \rightarrow \infty$. Thus, the specular component improves the BER at lower fading rates; for very high fading rates, the implicit diversity effect becomes dominant in diminishing the effect of fading.

The effect of the specular component on $P_{b}$ for a range of Rician factor, $K$, values is illustrated in Figure 4.3. As noted earlier, the BER decreases with $K$. However, a substantial gain in performance is observed for $K_{d B}>0 \mathrm{~dB}$. Since $K_{d B}=0 \mathrm{~dB}$ corresponds to equal powers in the fading and non-fading components of the channel, this behavior shows that a greater power in the non-faded signal component relative to the faded component diminishes the fading effect of the channel.

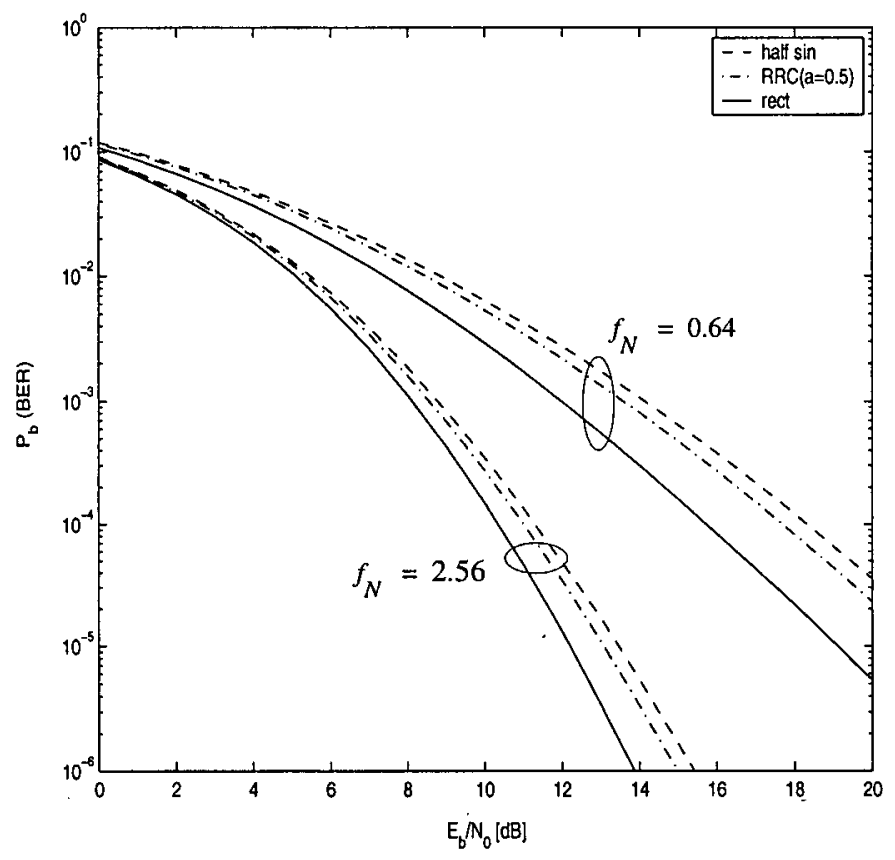

Figure 4.4 Effect of pulse shape in fast fading Rician channel, $K_{d B}=0 \mathrm{~dB}$ 
Unlike in very slow $\left(0.0<f_{N}<0.01\right)$ and nonfading conditions where the BER does not depend on the shape of the transmitted pulse, $p(t)$, the pulse shape does influence the BER in fast fading environments [8], [14]. This is illustrated in Figure 4.4 where the $P_{b}$ curves for three pulse shapes: rect, half sin and root raised cosine (RRC), are shown. The parameter ' $a$ ' refers to the roll-off factor for the RRC pulse. Due to less effective channel averaging for the half sin and RRC $(a>0)$ pulses, the BER, for the pulse shapes chosen, is found to be the lowest for the rect pulse.

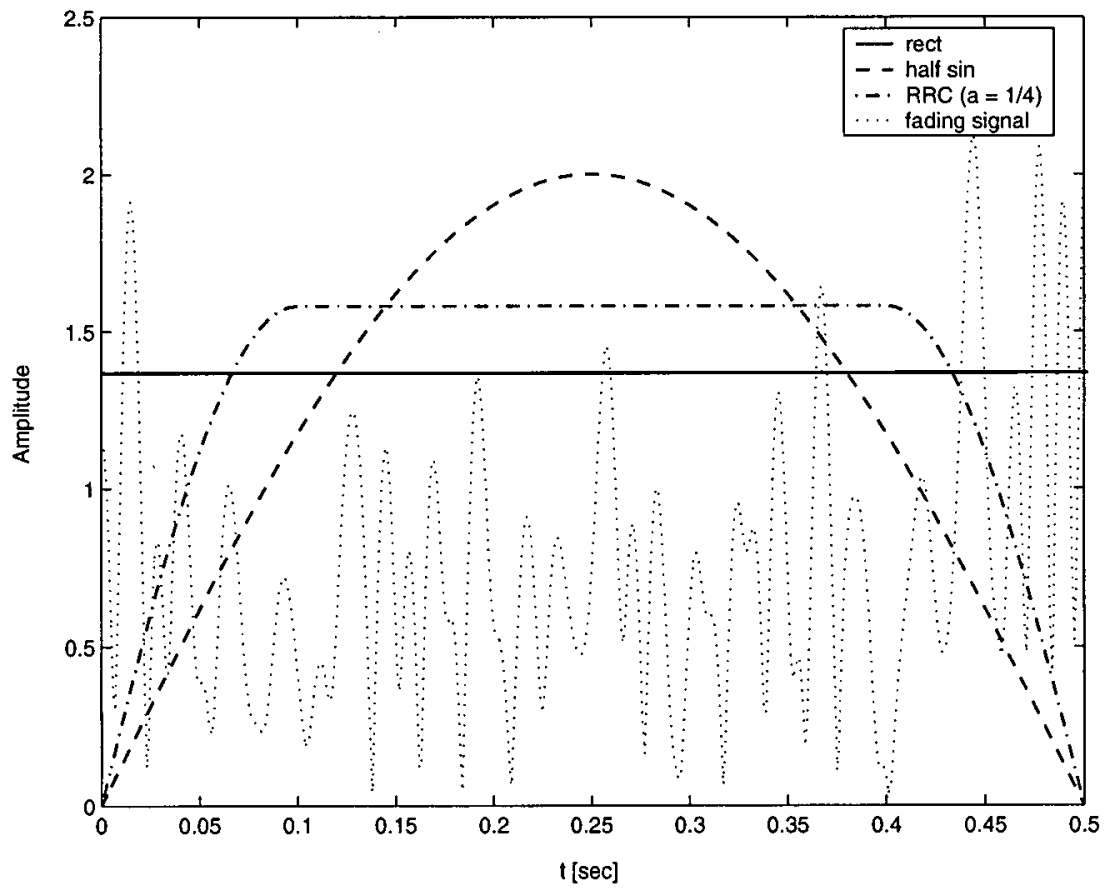

Figure 4.5 Channel averaging ability of different pulse shapes

Figure 4.5 shows the rect, half $\sin$ and RRC $(a=0.25)$ pulse shapes, all of the same pulse duration, $T_{p}=0.5 \mathrm{sec}$. A sample of a channel fading waveform is also shown. It can be seen that the amplitudes of the half sin and RRC pulse shapes are relatively smaller at the beginning and end of the pulses. 


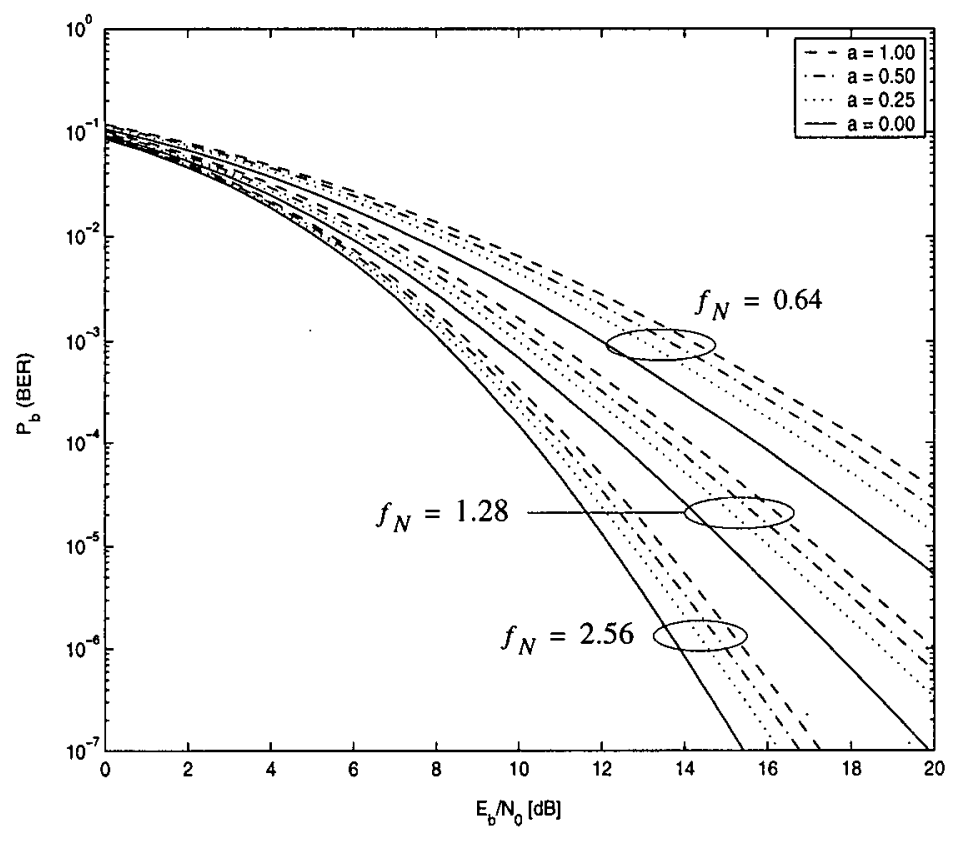

Figure 4.6 Effect of the roll-off factor on the system performance, $K_{d B}=0 \mathrm{~dB}$

It is important to understand the basis on which comparison among different pulse shapes is made, especially as it applies to the RRC pulse with varying roll-off factors. Since the normalized Doppler rate, $f_{N}=f_{d} T_{p}$ is a function of the total pulse duration, $T_{p}=T_{0}(1+a)$ where $T_{0}$ is the duration of a rect or half sin pulse, a roll-off factor of 1.0 (for instance) for the RRC pulse would correspond to twice the normalized Doppler rate for a rect pulse with the same Doppler frequency, $f_{d}$. Figure 4.6 shows how an increase in ' $a$ ' adversely affects the BER due to degradation in channel averaging ability of the pulse.

\subsection{Frequency-Selective Rician Fading}

The results for fast and slow fading Rician channel as two and three beam models are presented in this section. The curves are plotted for varying amount of delay (or overlap) and 
correlation between the beams and comparison is also made with respect to the Rician factor.

\subsubsection{Two-Beam Case}

MFB for the two-beam Rician channel, of which the first beam is the direct and the second the diffused beam, are illustrated in Figure 4.7 for both fast $\left(f_{N}=0.64\right)$ and very slow fading conditions. The curves are plotted for normalized delays of $0.0,0.5$ and 1.0 for the diffused beam which account for $100 \%$ overlap, $50 \%$ overlap and no overlap between the two beams. As expected the results for zero delay match with the flat fading BER and $P_{b}$ reduces with the decrease in the amount of overlapping (interference) between the two beams. Also the dB spread is found to be more in the slow fading case because in slow fading, diversity is achieved only from the extent of beam independence whereas in fast fading there is an additional advantage from the implicit diversity in each symbol.

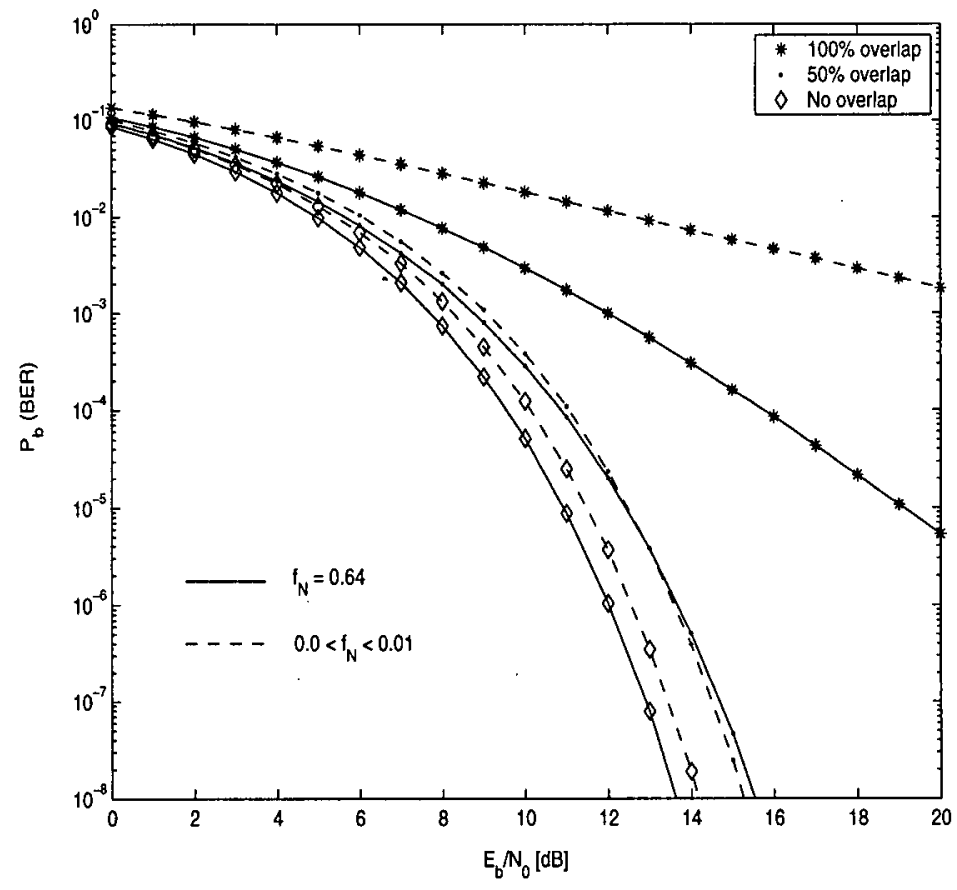

Figure 4.7 Two-beam frequency selective fading, $K_{d B}=0 \mathrm{~dB}$ 


\subsubsection{Three-Beam Case}

Figures 4.8 to 4.11 are plotted for the three-beam channel comprising of a direct/nonfaded beam and two diffused/faded beams. The curves are presented for varying normalized delays, $\sigma$, and correlation coefficients, $\rho$, between the two faded components assuming no overlapping with the non-faded component. The correlation [26] between the fading processes in the two beams is implemented as follows: two (independent) sets of fading samples are generated using the fading simulator. Samples from one of the sets are then scaled by the factor $\sqrt{1-\rho^{2}}$ and a fraction $(\rho)$ of the corresponding samples from the other set is added to the scaled samples. The two correlated sets of samples are then normalized independently.

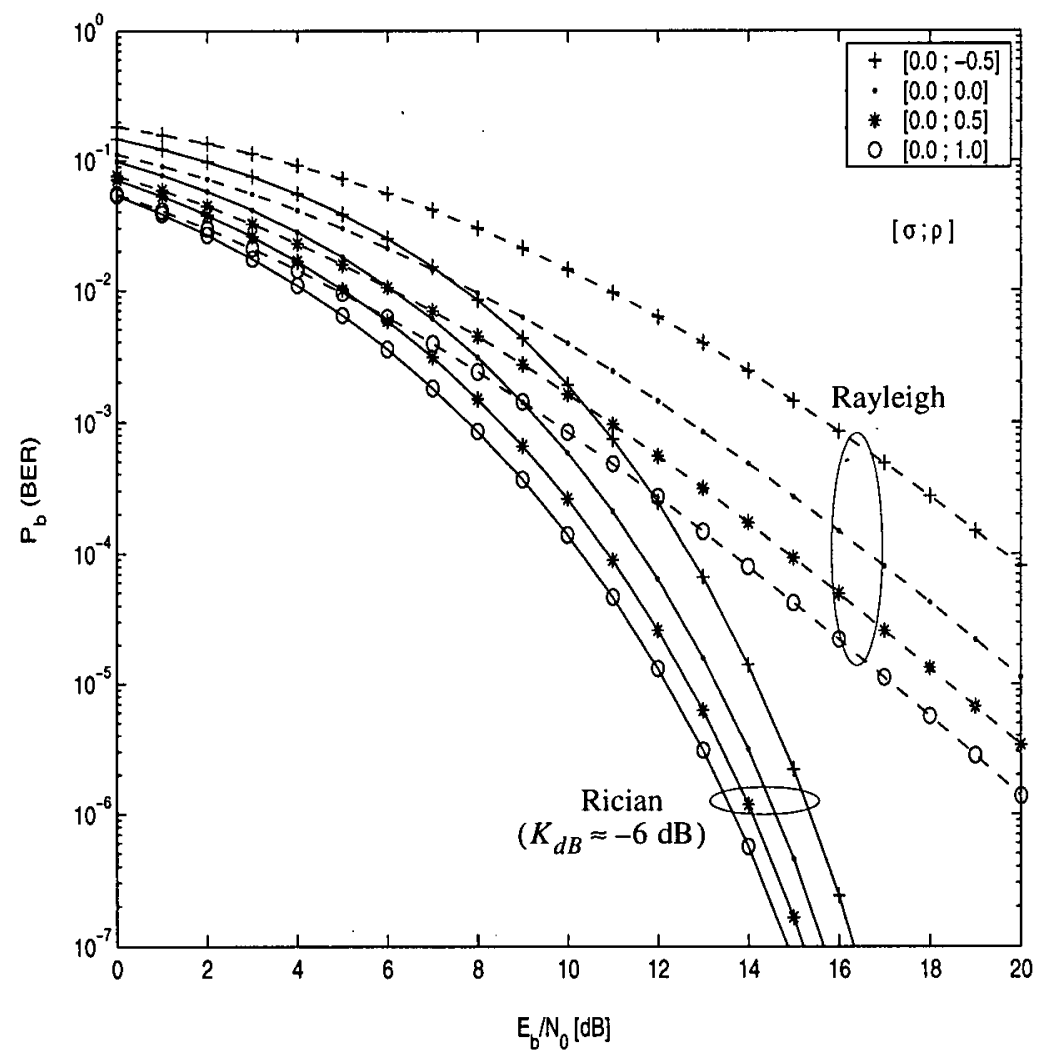

Figure 4.8 Three-beam frequency selective fast fading, $f_{N}=0.64$ and $\sigma=0$ 
Figures 4.8 and 4.9 refer to fast and slow fading conditions with no delay between the two diffused beams i.e. $\sigma=0$. In both cases it has been found that an increase in $\rho$ results in an improvement in system performance due to an increase in the received signal strength when the beams tend to be correlated and co-phased. At the other extreme, a condition of $[0.0 ;-1.0]$ would result in total cancellation of the diffused beams and reception of just the non-faded signal component. Although not shown in the figures for the sake of clarity, $[0.0 ;-1.0]$ corresponds to a constant BER of 0.5 for the Rayleigh channel; for the Rician channel since $K_{d B} \approx-6 \mathrm{~dB}$ corresponds to a power of 0.2 (for a unity gain channel) in the direct beam, $P_{b}=\frac{1}{2} \operatorname{erfc}\left(\sqrt{\frac{E_{b}}{5 N_{0}}}\right)$. Comparing Figures 4.8 and 4.9, the gain (obtained with an increase in $\rho$ ) in the latter is seen to be reduced. This is because in fast fading there is diversity within a single pulse.

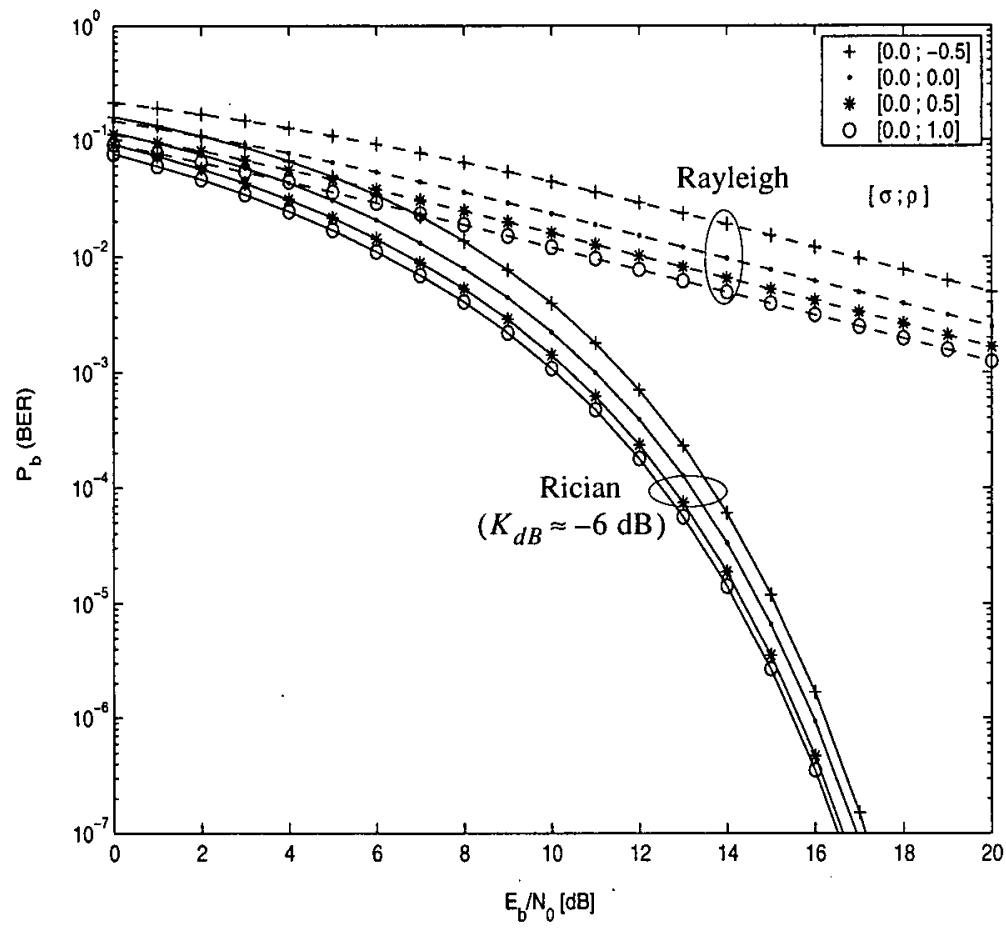

Figure 4.9 Three-beam frequency selective very-slow fading, $\sigma=0$ 
Figures 4.10 and 4.11 illustrate the BER curves assuming a delay between the diffused beams. The curves corresponding to the cases $[1.0 ;-1.0]$ and $[1.0 ; 1.0]$ (not shown in figures) coincide since the filter can resolve the two beams independently. In contrast to $\sigma=0$ case, a gain in performance is observed (especially for $\sigma \rightarrow 1$ ) with lesser correlation $(|\rho| \rightarrow 0)$ between the two beams. This occurs due to the diversity effect achieved when the fading in the two beams is uncorrelated. Also the gain is less for the fast fading case due to the inherent diversity present within a single pulse.

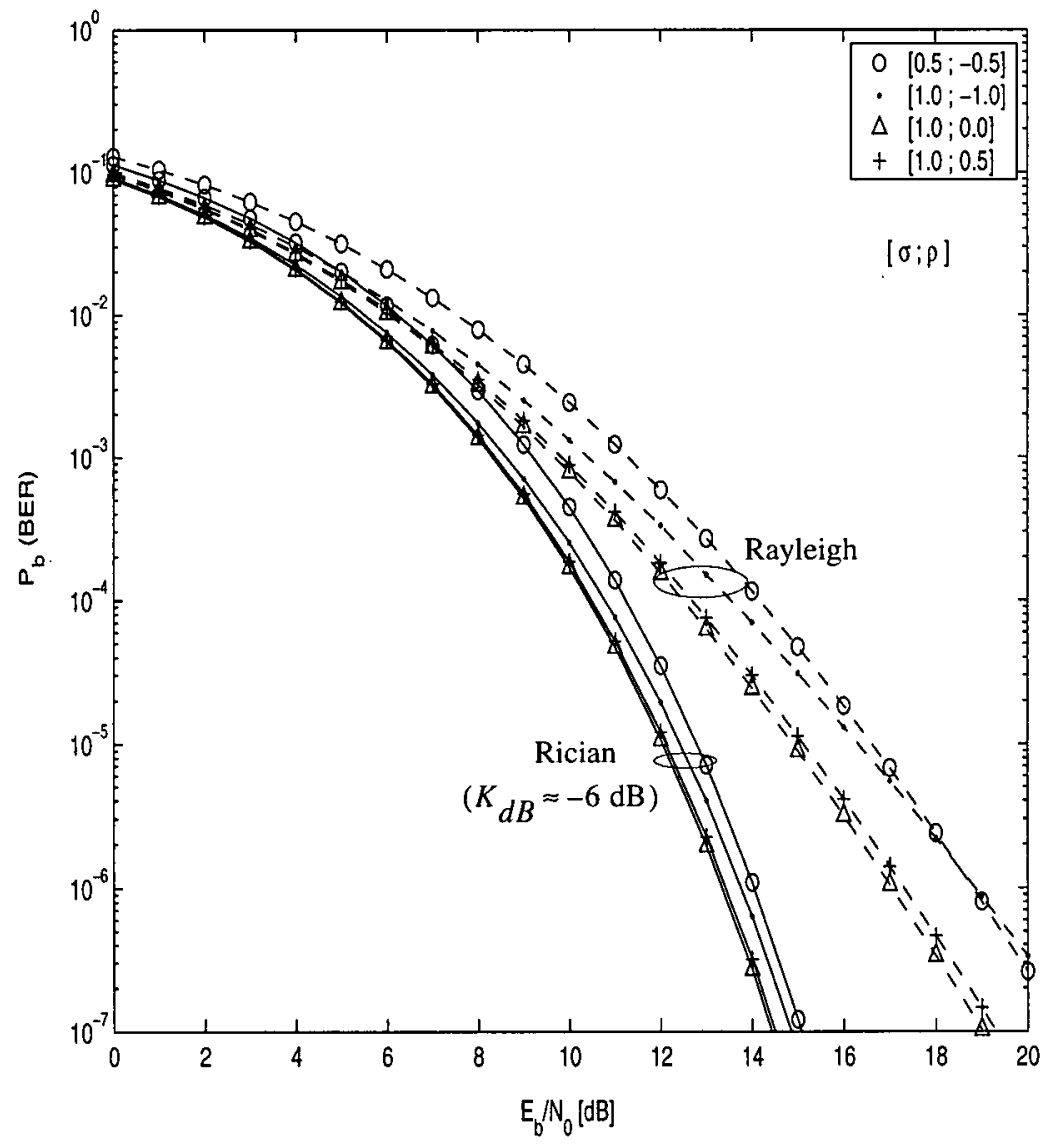

Figure 4.10 Three-beam frequency selective fast fading, $f_{N}=0.64$ and $\sigma>0$ 


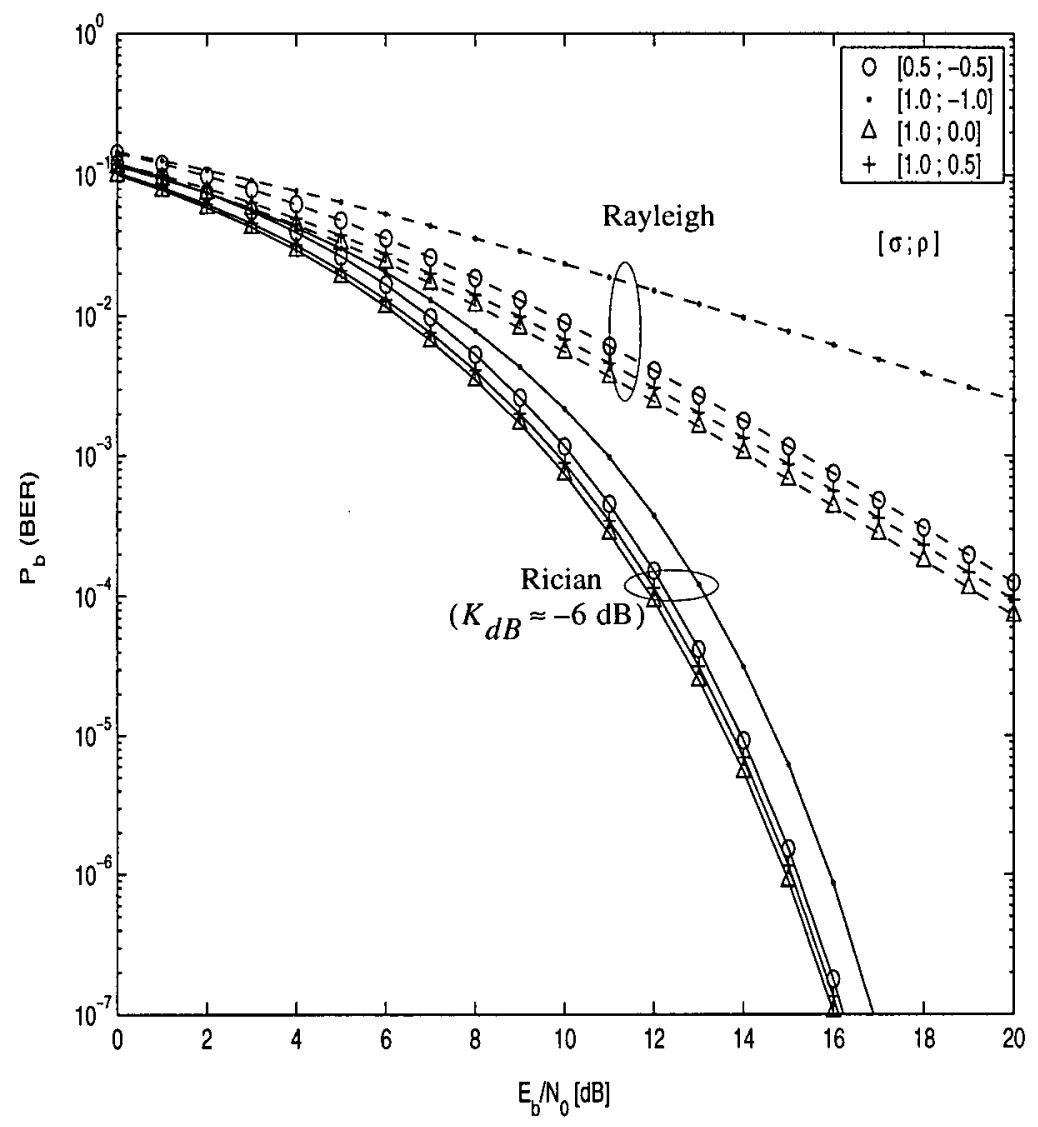

Figure 4.11 Three-beam frequency selective very-slow fading, $\sigma>0$

Another model of the three-beam channel is considered in Figures 4.12 to 4.16 for frequency selective fading. It is assumed that the non-faded beam and one of the faded beams coincide. The curves are plotted as a function of the normalized delay $(0 \leq \sigma \leq 1)$ and correlation coefficient $(-1 \leq \rho \leq 1)$ between the two faded beams.

Figure 4.12 displays the BER curves for a fast fading Rician channel with equal channel gain for the faded and the non-faded beams. In general, gain in BER performance is observed with an increase in $\sigma$. The case $[0.0 ;-1.0]$ is equivalent to transmitting the signal through a channel comprising of only the direct path of half the total strength of the Rician ( $\left.K_{d B}=0 \mathrm{~dB}\right)$ channel. 


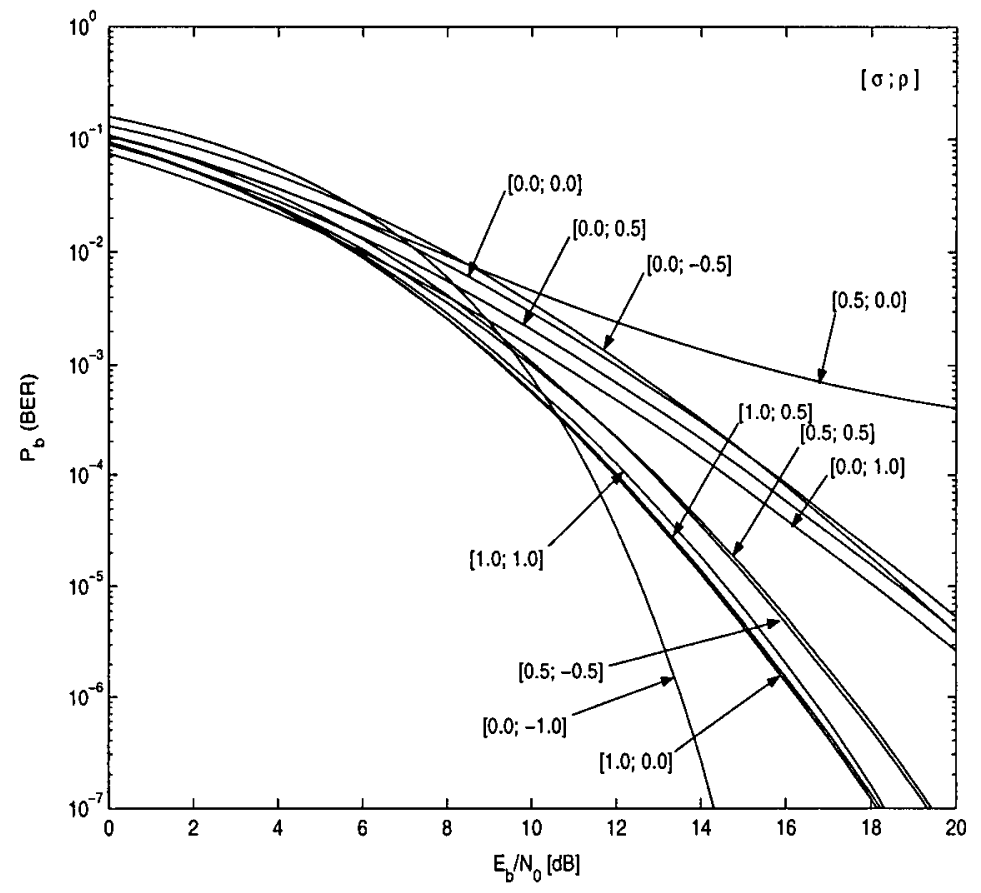

Figure 4.12 Three-beam fast fading, $K_{d B}=0 \mathrm{~dB}$ and $f_{N}=0.64$

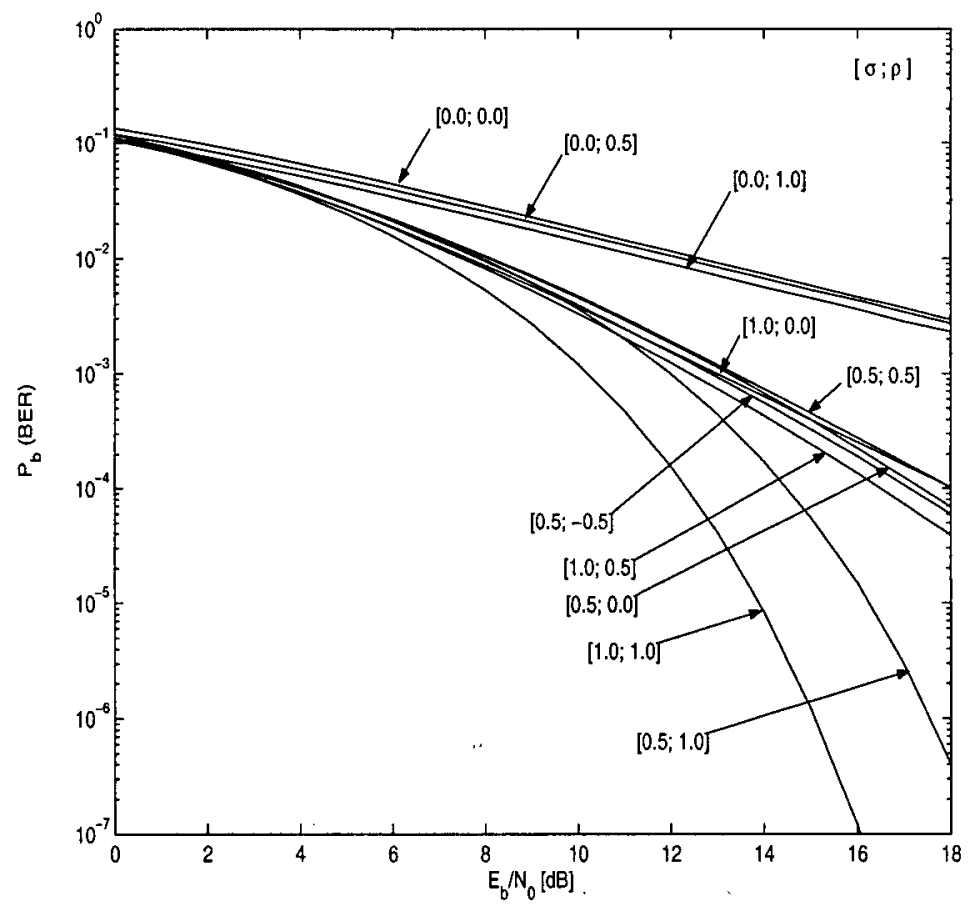

Figure 4.13 Three-beam slow fading, $K_{d B}=0 \mathrm{~dB}$ 
Figure 4.13 depicts BER for slow fading Rician channel. The variations of $P_{b}$ with [ $\left.\sigma ; \rho\right]$ are similar to the fast fading case except for a few cases of which $[1.0 ; 0.0]$ is particularly noteworthy. Since in a slow fading channel, the signal amplitude does not vary within the symbol duration, interference between the direct beam and the coinciding diffused beam results in a greater loss in the received signal energy when they tend to be out of phase. On comparing $[1.0 ; 1.0]$ with $[1.0 ; 0.0]$, in contrast to the fast fading case, we find the former has a lower BER and the gain $>5 \mathrm{~dB}$ at $P_{b}=10^{-4}$.

To facilitate comparison and in particular to observe the trends in system performance as a function of the Rician factor in fast and slow fading conditions, curves are plotted for four combinations of $[\sigma ; \rho]$

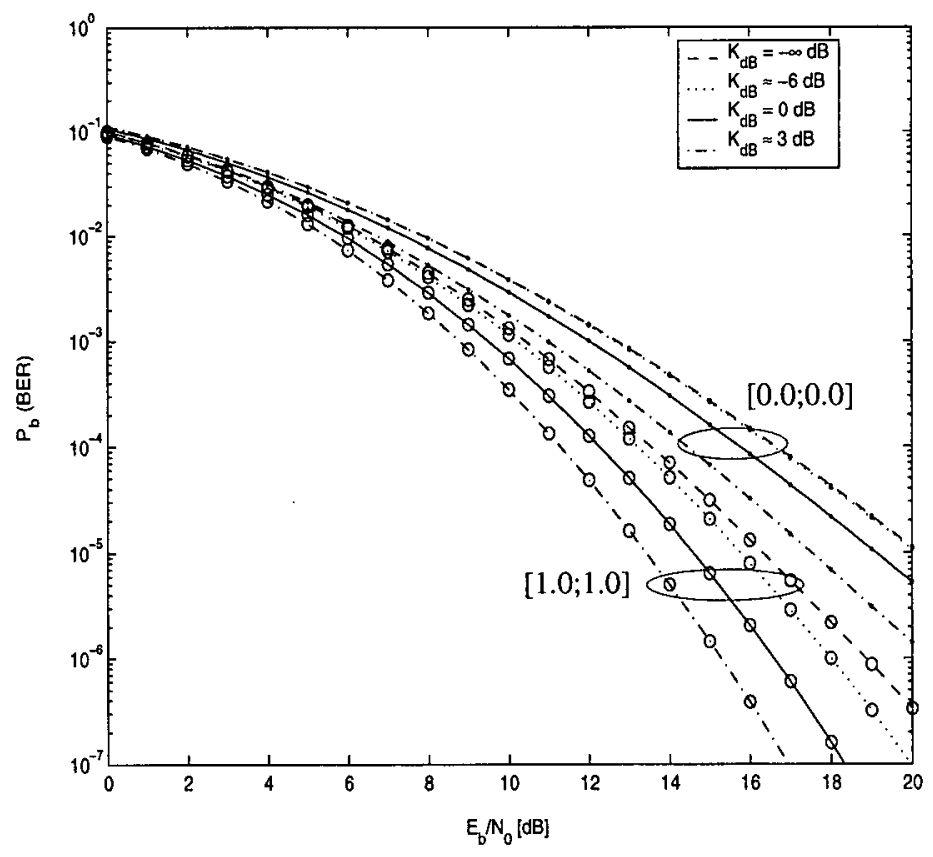

Figure 4.14 Three-beam fast fading as a function of $K$; $[\sigma ; \rho]:[0.0 ; 0.0],[1.0 ; 1.0]$

In Figure 4.14, the gain is seen to be more for $[1.0 ; 1.0]$ than for $[0.0 ; 0.0]$ for small $K$ 
values because of the lower received signal strength in $[0.0 ; 0.0]$ when the fading in the two coincident diffused beams is independent.

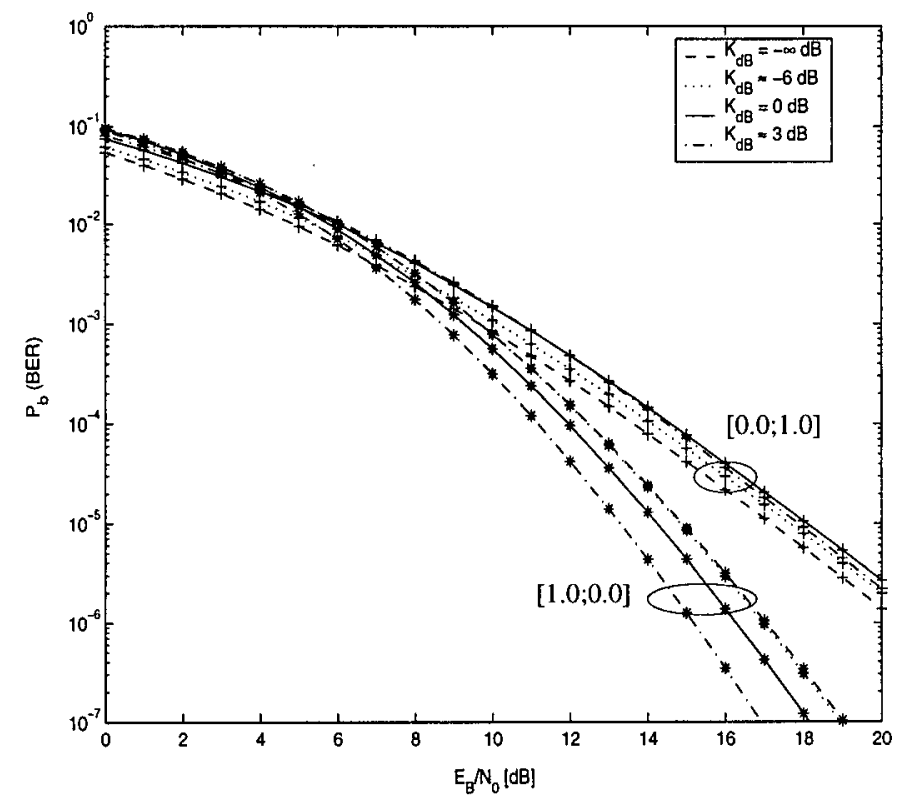

Figure 4.15 Three-beam fast fading as a function of $K ;[\sigma ; \rho]:[0.0 ; 1.0],[1.0 ; 0.0]$

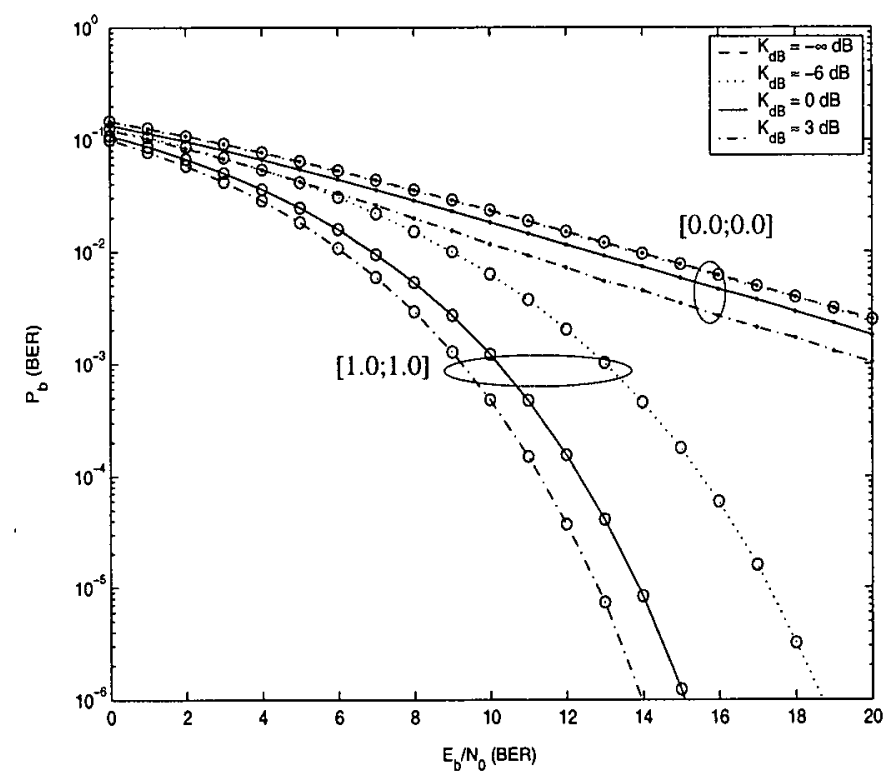

Figure 4.16 Three-beam slow fading as a function of $K ;[\sigma ; \rho]:[0.0 ; 0.0],[1.0 ; 1.0]$ 
In Figure 4.15 , the case $[0.0 ; 1.0]$ seems to follow an opposite trend i.e. $P_{b}$ increases as the Rician factor is increased from $K_{d B}=-\infty \mathrm{dB}$ to $K_{d B}=0 \mathrm{~dB}$. This occurs because at low Rician factors, i.e. $K_{d B}<0 \mathrm{~dB}$, when the strength of the direct beam is lower than that of the diffused beams, the faded component influences the received signal amplitude more than the nonfaded component; a decrease in the power of the diffused beam, due to an increase in $K$, tends to adversely affect the system performance. However for $K_{d B}=3 \mathrm{~dB}$, the non-faded signal dominates and a gain is observed with further increase in $K$.

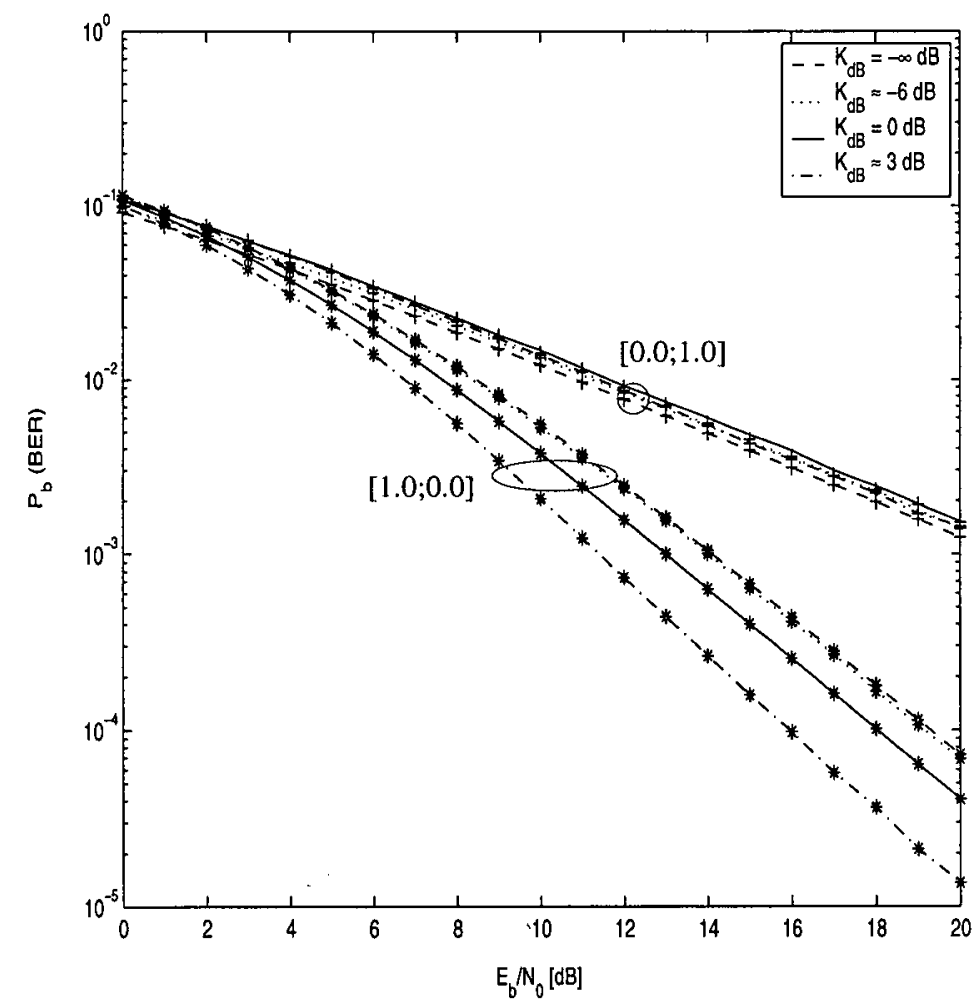

Figure 4.17 Three-beam slow fading as a function of $K$; $[\sigma ; \rho]:[0.0 ; 1.0],[1.0 ; 0.0]$

The variations of $P_{b}$ with $[\sigma ; \rho]$ for the slow fading channel as observed in Figures 4.16 and 4.17 are found to be similar to the fast fading case. 


\subsection{Imperfect channel estimation}

We have analyzed the system behavior when the receiver consists of a correlator demodulator which is perfectly matched to the fading channel. The case when the receive filter makes some error in estimating the channel variations is considered in the present section.

It is assumed that in estimating the channel fading samples, the receiver is liable to make random errors which are Gaussian distributed with zero mean and variance, $\sigma_{e}^{2}$, proportional to the average power, $\alpha^{2}$, of the faded beam. We study the performance for flat fading Rician $\left(K_{d B}=0 \mathrm{~dB}\right)$ and Rayleigh channels, each with a unit power gain.

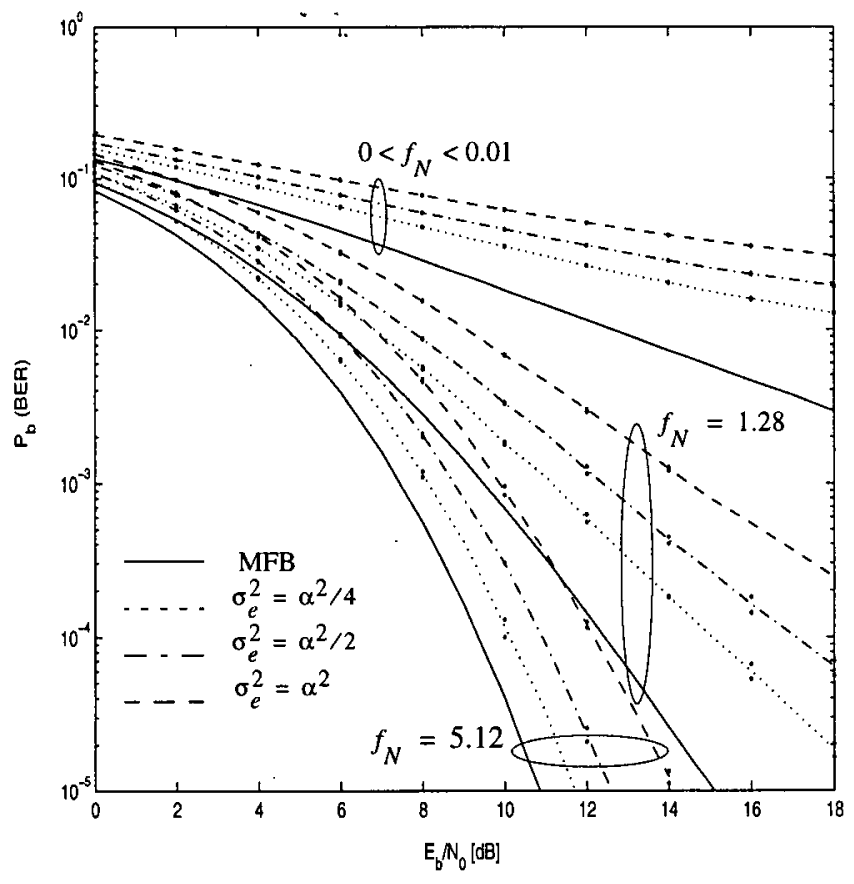

Figure 4.18 Performance degradation in a flat fading Rician channel

By modifying $\sigma_{e}^{2}$, the BER curves, obtained from simulations with $99 \%$ confidence intervals within $\pm 7 \%$ of the mean value, are plotted for varying degree of error in estimation. A 
rect pulse shape is used in the simulations. The mis-matched signal in the receiver is simulated by adding a complex Gaussian random process, with i.i.d. real and imaginary parts each with power spectral density $\sigma_{e}^{2} / 2$, to the fading waveform. In order to study the sensitivity of the (non-ideal) filter under different fading conditions, results for both fast and very-slow fading channels are shown.

The degradation in system performance for the Rician and Rayleigh channels as a function of $\sigma_{e}^{2}$ is shown in Figures 4.18 and 4.19 respectively. Since $\sigma_{e}^{2}$ depends on the power of the faded beam, the degradation in performance tends to be more for the Rayleigh channel.

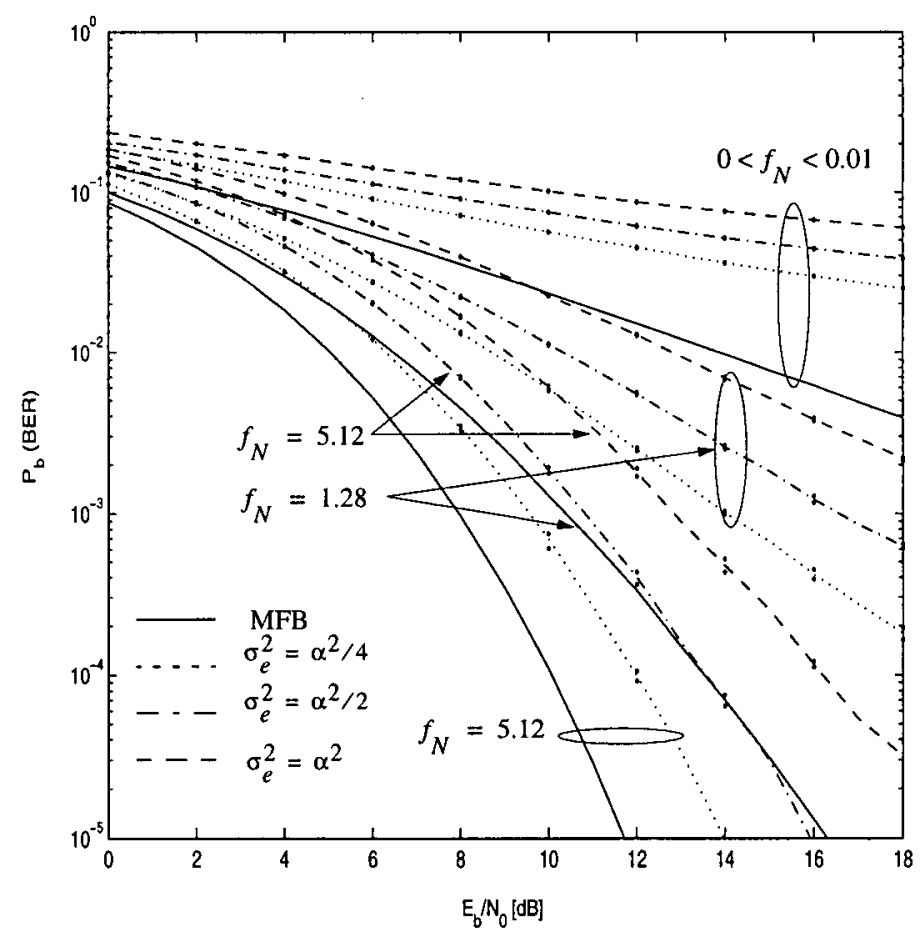

Figure 4.19 Performance degradation in a flat fading Rayleigh channel

The following plots (Figures 4.20 to 4.23) illustrate the influence of error in estimating the amplitude and phase, individually, on the BER. 


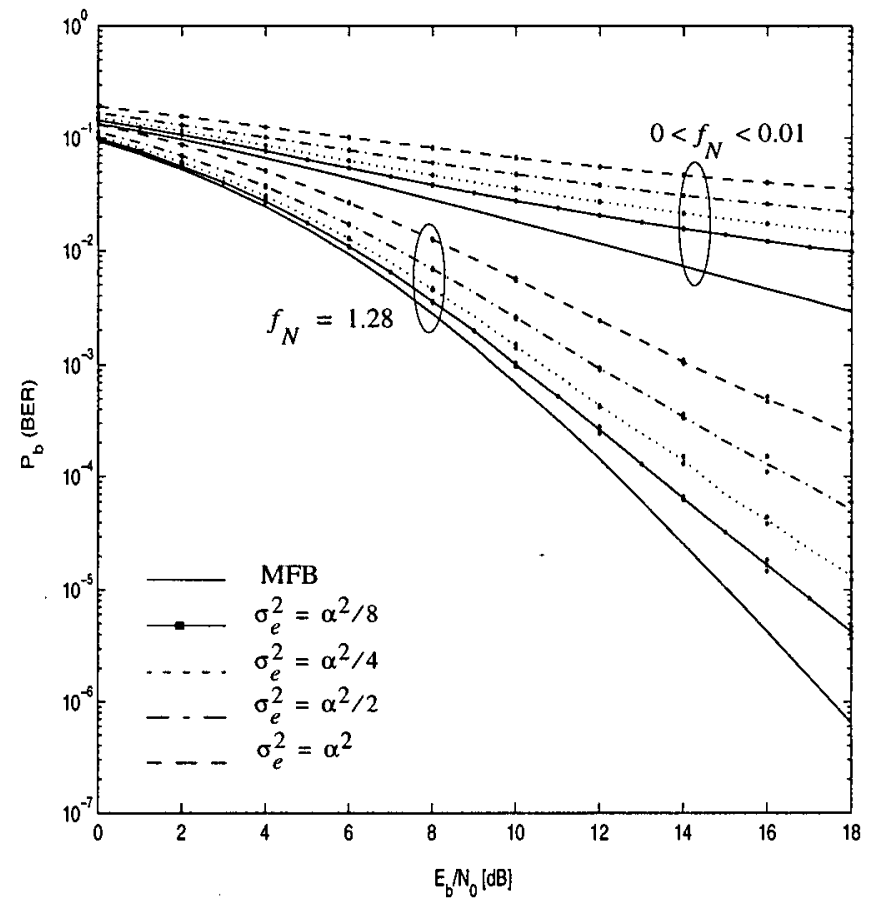

Figure 4.20 Effect of mis-match in phase on the BER in a Rician channel

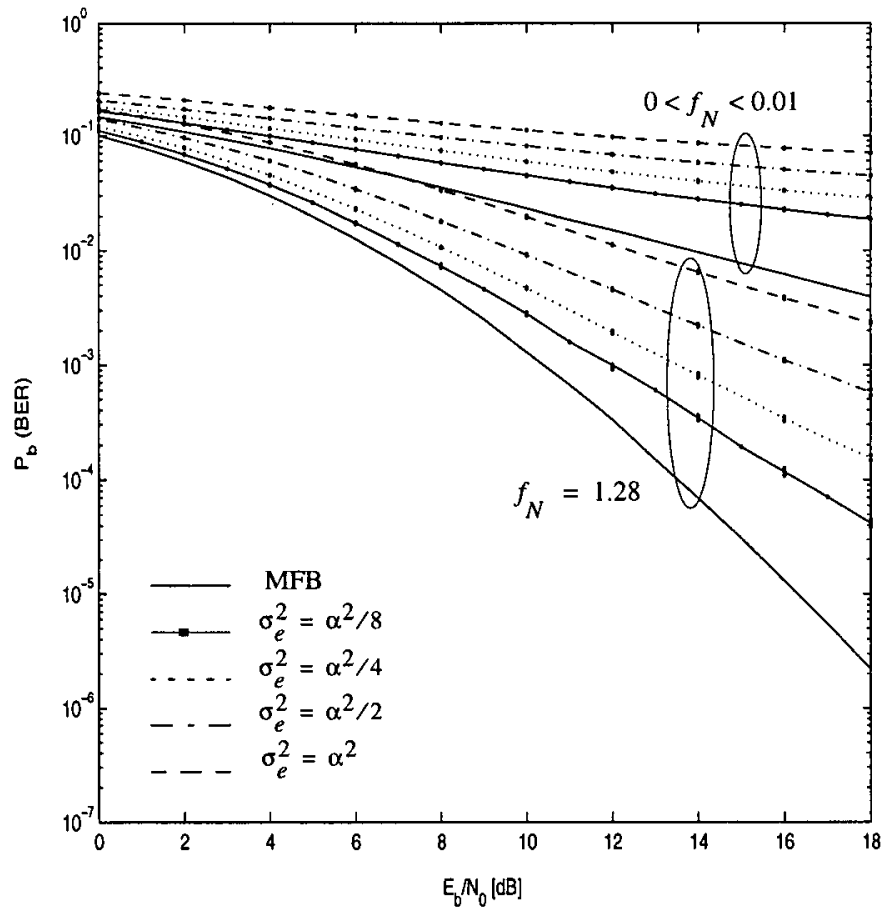

Figure 4.21 Effect of mis-match in phase on the BER in a Rayleigh channel 
Figures 4.20 and 4.21 correspond to the BER for the case when the filter is unable to correctly trace the phase of the fading waveform. Although, for all the values of $\sigma_{e}^{2}$ chosen, the BER for the fast fading case is found to be lower than that for the slow fading case, the $\mathrm{dB}$ spread corresponding to $f_{N}=1.28$ is found to be considerably greater than the dB spread corresponding to $0<f_{N}<0.01$. A similar observation can be made in Figures 4.22 and 4.23 which correspond to the effect of error in estimating the amplitude of the fading samples.

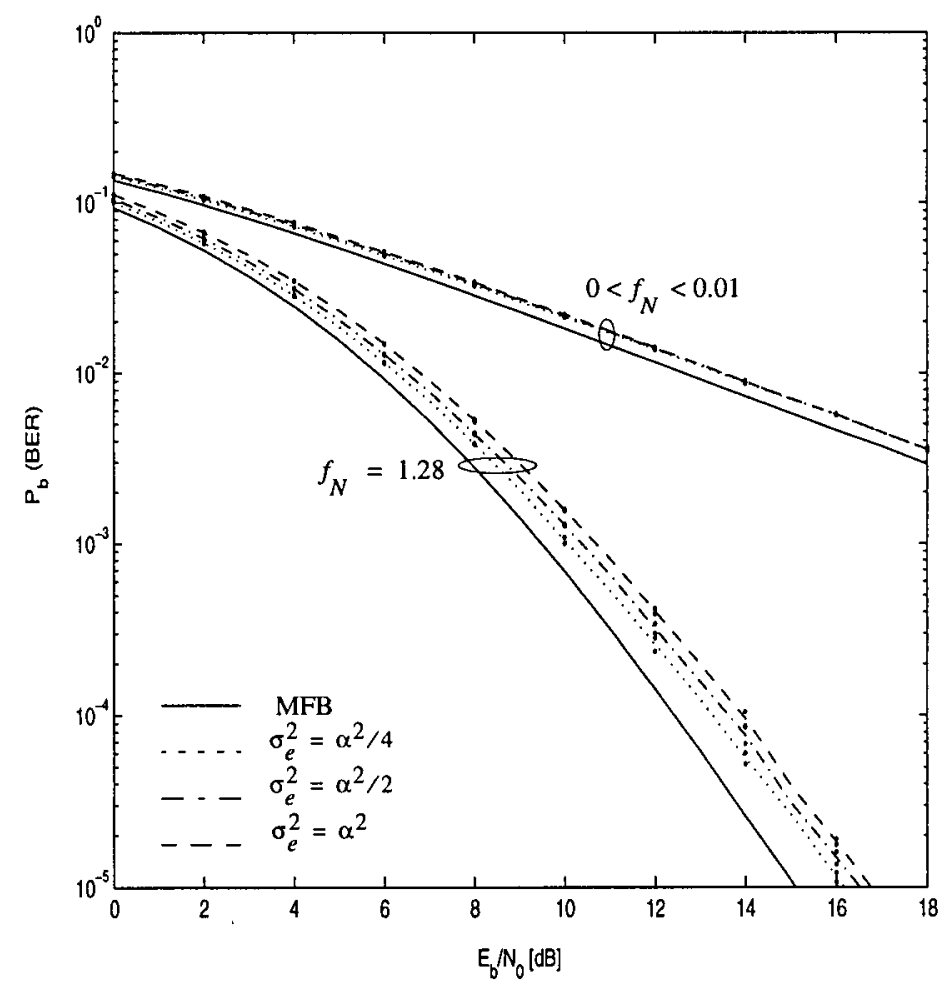

Figure 4.22 Effect of mis-match in amplitude on the BER in a Rician channel

Caution should be exercised in deriving conclusions from a comparison of the figures showing the effects of phase and amplitude estimation errors since the curves presented correspond to the specific model assumed in the simulations. The BER curves which are plotted 
as a function of $\sigma_{e}^{2}$ reveal a greater degradation in receiver performance caused by imperfect phase estimation than that due to imperfect amplitude estimation. The plots do not imply that in general the receivers are more sensitive to phase errors than to amplitude errors.

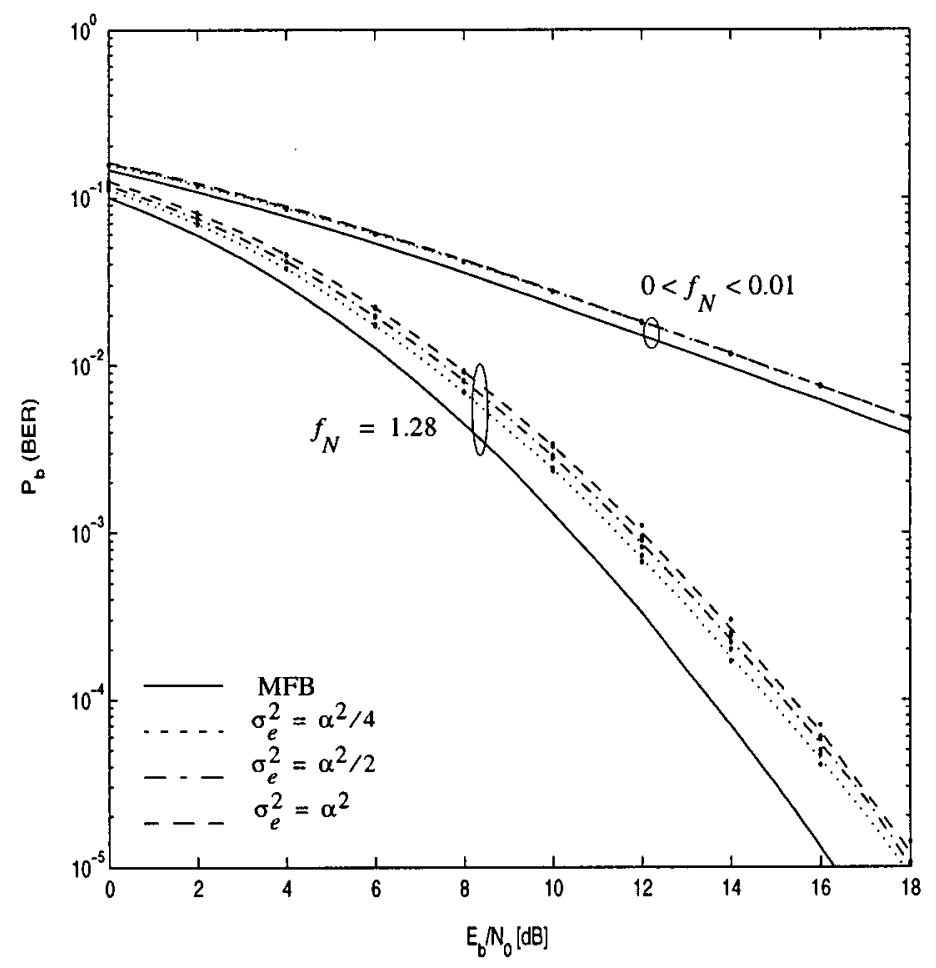

Figure 4.23 Effect of mis-match in amplitude on the BER in a Rayleigh channel

The calculations corresponding to the mean square error (MSE) in amplitude estimation and mean error in phase estimation are shown in Appendix D. 


\section{Chapter 5 Conclusions and Suggestions for Future Work}

\subsection{Conclusions}

Matched filter bounds for fast fading Rician channels have been derived to establish lower bounds on the BER for uncoded BPSK modulation over flat and frequency-selective fading channels. The analysis holds for any value of the normalized Doppler rate, $f_{N}$. The BER performance, in general, is found to improve with an increase in the fading rate due to the inherent diversity present within a single pulse. All the analytical results were verified to be in close agreement with simulation results.

Unlike the Rayleigh fading case where the statistical distribution of the (received) random signal component, after diagonalizing the covariance matrix of the fading samples, is given by the central chi squared distribution, the Rician case is more complex. The new random signal component is a sum of three terms: a constant term, a scaled version of the signal component encountered in the Rayleigh case and a term which is dependent on the second term. The distribution was derived by separating the i.i.d. real and imaginary parts of the uncorrelated complex Gaussian random variables encountered in the second term and grouping all the real components and imaginary components separately; the random signal component can then be expressed as a sum of a constant term and a random variable with a non-central chi squared distribution.

A comparison between the BER curves for the Rayleigh and the Rician channels, as a function of $f_{N}$ in flat fading suggests that under slow fading conditions, the performance gain is attributed mainly to the presence of the direct/specular component in the Rician channel whereas when $f_{N}$ increases without limit, the gap between the two tends to reduce and both the curves 
finally converge with the MFB for the non-fading AWGN channel. The latter effect shows the dominant role of the fading rate in lowering the BER in fast fading conditions. In general, the BER performance improves with an increase in $K$; little gain is observed when the ratio of the powers of the direct and diffused beams is less than $-6 \mathrm{~dB}$. The system performance was also studied for different transmitted pulse shapes: it was found that the pulse shape affects the BER significantly when the channel exhibits fast fading. This is consistent with earlier results for the Rayleigh case [8], [14]. Results for the frequency selective channel, derived for the general case of correlated scattering, were also examined. In two-beam frequency selective fading, particularly for slow fading, the BER is very sensitive to the degree of beam overlap.

The BER performance of a receive filter whose impulse response is not perfectly matched to the channel fading waveform was studied through simulation. It was observed that, for the specified model, the system degradation was higher for phase mismatches than for amplitude mismatches for a given error variance.

\subsection{Suggestions for Future Work}

1. In this thesis, the pdf, $f_{Y}(y)$, of the signal component, $Y$, is obtained from its characteristic function, $\Psi_{Y}(v)$, through numerical integration. To the best of the author's knowledge, no computationally effective closed form expression of the pdf of unequally weighted noncentral chi squared random variables is available. To avoid numerical approximation errors and computational complexity, it would be beneficial to obtain a closed form expression for $f_{Y}(y)$

2. In the present work, frequency selectivity in the channel is obtained by assuming a double 
spike model for the delay power spectrum of the fading channel with arbitrary correlation between the fading waveforms. The analysis is limited to a single branch system i.e. without explicit diversity. Deriving the MFB's for a multibranch system would be a useful extension to this work.

3. MFB's for both flat and frequency selective fading in a rapidly time-varying Rician channel have been derived assuming BPSK modulation. The same for any PAM(ASK) scheme can also be obtained with some minor modifications; the new (variable) threshold boundaries of the decision maker would be a function of the received signal component. Deriving the error bounds other modulation schemes can prove to be a little challenging especially when the transmitted pulse, $p(t)$, is a complex entity.

4. The BER corresponding to imperfect channel estimation were obtained by simulations and presented in Section 4.4. The theoretical analysis for the same is complicated since the additive Gaussian error in the matched signal, i.e. the impulse response of the receive filter, does not correspond exactly to the assumption that the noise component in the decision variable is Gaussian. 


\section{Appendix A : MFB (slow fading $0<f_{N}<0.01$ )}

The impulse response of a slow fading frequency-selective Rician channel assuming reception of two faded (Rayleigh) components in addition to the direct one, i.e. three-beam channel, when one of the faded beams and the non-faded beam coincide, is given as

$$
\begin{aligned}
z(t, \tau) & =\alpha_{0} \delta(\tau)+Z_{1} \delta(\tau)+Z_{2} \delta(\tau-\sigma) \\
& =\left(\alpha_{0}+Z_{1}\right) \delta(\tau)+Z_{2} \delta(\tau-\sigma)
\end{aligned}
$$

where $Z_{1}$ and $Z_{2}$ are complex Gaussian random variables with i.i.d. real and imaginary parts $Z_{n}=U_{n}+j V_{n}, \quad n=1,2$ and $\alpha_{0}^{2}$ is the power of the direct/specular path. Using the following expansion, i.e.

$$
\left|Z_{1}+Z_{2}\right|^{2}=\left|Z_{1}\right|^{2}+\left|Z_{2}\right|^{2}+2 \operatorname{Re}\left[Z_{1}\right] \operatorname{Re}\left[Z_{2}\right]+2 \operatorname{Im}\left[Z_{1}\right] \operatorname{Im}\left[Z_{2}\right]
$$

the corresponding signal component $Y$ can be written as

$$
Y=\alpha_{0}^{2}+\left|Z_{1}\right|^{2}+\left|Z_{2}\right|^{2}+2 \alpha_{0} U_{1}+2 \alpha_{0} U_{2} k+2 U_{1} U_{2} k+2 V_{1} V_{2} k
$$

where $k=\int_{\sigma}^{T_{p}} p(t) p(t-\sigma) d t$ is a constant symbolizing the amount of pulse overlap (between the two diffused beams) during signal reception.

For the special case when the fading in the delayed and non-delayed beams is uncorrelated and there is no overlapping i.e. $\sigma \geq T_{p}, k=0$ and (A.3) simplifies to

$$
Y=\left(U_{1}+\alpha_{0}\right)^{2}+U_{2}^{2}+V_{1}^{2}+V_{2}^{2}
$$

$Y$ is thus stated as a sum of non-central chi-squared degree 4 random variable. The cdf of $Y$ is 
given as [27]

$$
F_{Y}(y)=\sum_{n=0}^{\infty} C_{n} P_{r}\left(\chi_{4+2 n}^{2} \leq \frac{y}{\beta}\right)
$$

where $\beta=\frac{E\left[\left|Z_{1}\right|^{2}\right] E\left[\left|Z_{2}\right|^{2}\right]}{E\left[\left|Z_{1}\right|^{2}\right]+E\left[\left|Z_{2}\right|^{2}\right]}$ is a suitably chosen constant [7]. From [28], we have

$$
P_{r}\left(\chi_{4}^{2}+2 n \leq \frac{y}{\beta}\right)=1-\exp \left(-\frac{y}{2 \beta}\right) \cdot \sum_{k=0}^{n+1} \frac{1}{k !}\left(\frac{y}{2 \beta}\right)^{k}
$$

Substituting (A.6) into (A.5) and computing the pdf of $Y$ by differentiating $F_{Y}(y)$, we obtain

$$
\begin{aligned}
f_{Y}(y) & =\frac{d}{d y} F_{Y}(y)=\sum_{n=0}^{\infty} C_{n}\left[\frac{1}{2 \beta} e^{-\frac{y}{2 \beta}} \sum_{k=0}^{n+1} \frac{1}{k !}\left(\frac{y}{2 \beta}\right)^{k}-e^{-\frac{y}{2 \beta}} \sum_{k=1}^{n+1} \frac{1}{(k-1) ! \frac{1}{2 \beta}}\left(\frac{y}{2 \beta}\right)^{k-1}\right] \\
& =\sum_{n=0}^{\infty} C_{n} \frac{1}{2 \beta} e^{-\frac{y}{2 \beta}}\left[\sum_{k=0}^{n+1} \frac{1}{k !}\left(\frac{y}{2 \beta}\right)^{k-1}\left(\frac{y}{2 \beta}-k\right)\right] .
\end{aligned}
$$

The BER is thus given by (3.3.13)

$$
\begin{aligned}
P_{b} & =\frac{1}{2} \int_{0}^{\infty} \operatorname{erfc}\left(\sqrt{\frac{E_{b} y}{N_{0}}}\right) \cdot \sum_{n=0}^{\infty} C_{n} \frac{1}{2 \beta} e^{-\frac{y}{2 \beta}}\left[\sum_{k=0}^{n+1} \frac{1}{k !}\left(\frac{y}{2 \beta}\right)^{k-1}\left(\frac{y}{2 \beta}-k\right)\right] d y \\
& =\frac{1}{2} \int_{0}^{\infty} \operatorname{erfc}\left(\sqrt{\frac{E_{b} y}{N_{0}}}\right) \cdot F_{Y}^{\prime}(y) d y .
\end{aligned}
$$

Integrating (A.8) by parts and simplifying 


$$
\begin{aligned}
P_{b} & =\frac{1}{2}\left(\left.\operatorname{erfc}\left(\sqrt{\frac{E_{b} y}{N_{0}}}\right) F_{Y}(y)\right|_{0} ^{\infty}-\int_{0}^{\infty} F_{Y}(y) d\left[\operatorname{erfc}\left(\sqrt{\frac{E_{b} y}{N_{0}}}\right)\right]\right) \\
& =\frac{1}{2} \sqrt{\frac{E_{b}}{N_{0} \pi}} \int_{0}^{\infty} F_{Y}(y) \exp \left(-\frac{E_{b} y}{N_{0}}\right) y^{-1 / 2} d y \\
& =\frac{1}{2} \sqrt{\frac{E_{b}}{N_{0} \pi}} \cdot \sum_{n=0}^{\infty} C_{n} \int_{0}^{\infty}\left[1-\exp \left(-\frac{y}{2 \beta}\right) \cdot \sum_{k=0}^{n+1} \frac{1}{k !}\left(\frac{y}{2 \beta}\right)^{k}\right] \exp \left(-\frac{E_{b} y}{N_{0}}\right) y^{-1 / 2} d y
\end{aligned}
$$

Knowing that [23]

$$
\int_{0}^{\infty} \exp (-q y) y^{-1 / 2} d y=\sqrt{\frac{\pi}{q}}
$$

and

$$
\int_{0}^{\infty} e^{-q y} y^{n-\frac{1}{2}} d y=\sqrt{\pi} 2^{-n} q^{-n-\frac{1}{2}}(2 n-1) ! !, \quad n \geq 0
$$

equation (A.9) further simplifies to

$$
P_{b}=\frac{1}{2} \sum_{n=0}^{\infty} C_{n}\left[1-\sqrt{\frac{\mu-1}{\mu}}-\sum_{k=1}^{n+1} 2^{-2 k+1}\left(\begin{array}{c}
2 k-1 \\
k
\end{array}\right) \sqrt{\frac{\mu-1}{\mu}} \cdot\left(\frac{1}{\mu}\right)^{k}\right]
$$

where $\mu=1+2 \beta\left(E_{b} / N_{0}\right)$ is a constant. Assuming $E\left[\left|Z_{1}\right|^{2}\right]=E\left[\left|Z_{2}\right|^{2}\right]$, the coefficients $C_{n}$ are given as [27]

$$
\begin{aligned}
& C_{0}=\exp \left(-2 \alpha_{0}^{2} /\left(1-\alpha_{0}^{2}\right)\right) \\
& C_{n}=1 / n \cdot \sum_{j=0}^{n-1} b_{n-j} C_{j}, \quad n \geq 1 \\
& b_{n}= \begin{cases}2 \alpha_{0}^{2} /\left(1-\alpha_{0}^{2}\right), & n=1 \\
0, & n>1\end{cases}
\end{aligned}
$$




\section{Appendix B : MFB (fast fading $f_{N} \rightarrow \infty$ )}

We are interested in finding the limiting case of the variance of the signal component $Y$, $\sigma_{Y}^{2}$, as $f_{N} \rightarrow \infty$

For the flat-fading Rician channel, $Y$ is given by

$$
Y=\int\left|\alpha_{0}+\alpha_{1} z(t)\right|^{2} \cdot|p(t)|^{2} d t
$$

and its mean

$$
\begin{aligned}
E[Y] & =\int|p(t)|^{2} E\left[\left|\alpha_{0}+\alpha_{1} z(t)\right|^{2}\right] d t \\
& =\int|p(t)|^{2}\left(\alpha_{0}^{2}+\alpha_{1}^{2}\right) d t \\
& =\alpha_{0}^{2}+\alpha_{1}^{2}
\end{aligned}
$$

where we have used $E\left[|z(t)|^{2}\right]=1$. Accordingly we have

$$
\begin{aligned}
\sigma_{Y}^{2} & =E\left[Y^{2}\right]-(E[Y])^{2} \\
& =E\left[Y^{2}\right]-\left(\alpha_{0}^{2}+\alpha_{1}^{2}\right)^{2}
\end{aligned}
$$

Finding the second moment of $Y$

$$
E\left[Y^{2}\right]=\iint\left|p\left(t_{1}\right)\right|^{2}\left|p\left(t_{2}\right)\right|^{2} E\left[\left|\alpha_{0}+\alpha_{1} z\left(t_{1}\right)\right|^{2}\left|\alpha_{0}+\alpha_{1} z\left(t_{2}\right)\right|^{2}\right] d t_{1} d t_{2}
$$

Expanding the expectation term in the integral gives

$$
\begin{aligned}
\left|\alpha_{0}+\alpha_{1} z\left(t_{1}\right)\right|^{2}\left|\alpha_{0}+\alpha_{1} z\left(t_{2}\right)\right|^{2}= & \alpha_{0}^{4}+\alpha_{0}^{2} \alpha_{1}^{2}\left(\left|z\left(t_{1}\right)\right|^{2}+\left|z\left(t_{2}\right)\right|^{2}\right)+\alpha_{1}^{4}\left|z\left(t_{1}\right)\right|^{2}\left|z\left(t_{2}\right)\right|^{2} \\
& +2 \alpha_{0}^{3} \alpha_{1} \operatorname{Re}\left[z\left(t_{1}\right)\right]+2 \alpha_{0}^{3} \alpha_{1} \operatorname{Re}\left[z\left(t_{2}\right)\right] \\
& +2 \alpha_{0} \alpha_{1}^{3} \operatorname{Re}\left[z\left(t_{1}\right)\right]\left|z\left(t_{2}\right)\right|^{2}+2 \alpha_{0} \alpha_{1}^{3} \operatorname{Re}\left[z\left(t_{2}\right)\right]\left|z\left(t_{1}\right)\right|^{2} \\
& +4 \alpha_{0}^{2} \alpha_{1}^{2} \operatorname{Re}\left[z\left(t_{1}\right)\right] \operatorname{Re}\left[z\left(t_{2}\right)\right]
\end{aligned}
$$

and taking the expectation of both sides in (B.5) yields 


$$
E\left[\left|\alpha_{0}+\alpha_{1} z\left(t_{1}\right)\right|^{2}\left|\alpha_{0}+\alpha_{1} z\left(t_{2}\right)\right|^{2}\right]=\alpha_{0}^{4}+2 \alpha_{0}^{2} \alpha_{1}^{2}+\alpha_{1}^{4} E\left[\left|z\left(t_{1}\right)\right|^{2}\left|z\left(t_{2}\right)\right|^{2}\right]
$$

Substituting (B.6) into (B.4) and using [14]

$$
E\left[\left|z\left(t_{1}\right)\right|^{2}\left|z\left(t_{2}\right)\right|^{2}\right]=1+\rho^{2}
$$

where $\rho$ is the normalized cross-correlation between the two samples of the real or imaginary part of the process $z(t)$ [16]. Thus, we have

$$
E\left[Y^{2}\right]=\alpha_{0}^{4}+2 \alpha_{0}^{2} \alpha_{1}^{2}+\alpha_{1}^{4} \iint\left|p\left(t_{1}\right)\right|^{2}\left|p\left(t_{2}\right)\right|^{2}\left(1+\rho^{2}\right) d t_{1} d t_{2}
$$

Since $\rho$ can be viewed as the correlation of the fading process, from [15]

$$
\rho(\tau)=J_{0}\left(2 \pi f_{D} \tau\right)
$$

where $J_{O}(x)$ is the zeroth order Bessel function of the first kind and $f_{D}$ is the maximum Doppler frequency in Hertz. Applying the transformation $t_{1}-t_{2}=\tau$ and using (B.3), (B.8) and (B.9), the variance of $Y$ can be written as

$$
\sigma_{Y}^{2}=\alpha_{1}^{4} \iint\left|p\left(t_{1}\right)\right|^{2}\left|p\left(t_{1}-\tau\right)\right|^{2} J_{0}^{2}\left(2 \pi f_{D} \tau\right) d \tau d t_{1}
$$

Following the analysis in [14], we substitute the autocorrelation of the squared pulse magnitude which is given by

$$
R_{p}(\tau)=\int|p(t)|^{2}|p(t-\tau)|^{2} d t
$$

in (B.10) resulting in

$$
\sigma_{Y}^{2}=\alpha_{1}^{4} \int_{-T_{p}}^{T_{p}} R_{p}(\tau) \cdot J_{0}^{2}\left(2 \pi f_{D} \tau\right) d \tau
$$

The limiting variance of $Y$ as $f_{N} \rightarrow \infty$ can be obtained by taking the limit as $T_{p} \rightarrow \infty$. 
Writing $p(t)$ in terms of $u(t)$, a unit energy and unit duration pulse, i.e.

$$
p(t)=\frac{1}{\sqrt{T_{p}}} u\left(\frac{t}{T_{p}}\right)
$$

Substituting (B.13) in (B.11) and evaluating at $\tau=0$

$$
\begin{aligned}
R_{p}(0) & =\frac{1}{T_{p}^{2}} \int_{0}^{T_{p}} \mid u\left(\frac{t}{T_{p}}\right)^{4} d t \\
& =\frac{1}{T_{p}} \int_{0}^{1}|u(q)|^{4} d q \\
& =\frac{Q}{T_{p}}
\end{aligned}
$$

where $Q=\int_{0}^{1}|u(q)|^{4} d q$ is a constant. Since an autocorrelation function has it's maximum value at zero delay, it is convenient to write

$$
R_{p}(\tau) \leq \frac{Q}{T_{p}}
$$

which implies

$$
\sigma_{Y}^{2} \leq \frac{2 \alpha_{1}^{4} \int_{0}^{T_{p}} Q \cdot J_{0}^{2}\left(2 \pi f_{D} \tau\right) d \tau}{T_{p}}
$$

Taking the limit as $T_{p} \rightarrow \infty$ and applying L'Hopital's rule

$$
\lim _{T_{p} \rightarrow \infty} \sigma_{Y}^{2}=\lim _{T_{p} \rightarrow \infty} 2 \alpha_{1}^{4} Q J_{0}^{2}\left(2 \pi f_{D} T_{p}\right) d \tau=0
$$

Thus the variance of the signal component $Y$ approaches zero for any unit energy pulse as the normalized Doppler rate increases without limit. 


\section{Appendix C : Solving the Integral}

To evaluate the following integral encountered in Section 3.5.2:

$$
\begin{aligned}
P_{b} & =\frac{1}{2} \sum_{l=1}^{L_{d}} \sum_{m=1}^{r_{l}} \frac{B_{l m}}{(m-1) ! \lambda_{l}^{m}} \int_{\alpha_{0}^{2}}^{\infty} \operatorname{erfc}\left(\sqrt{\frac{E_{b} y}{N_{0}}}\right) \cdot\left(y-\alpha_{0}^{2}\right)^{m-1} \exp \left(-\frac{\left(y-\alpha_{0}^{2}\right)}{\lambda_{l}}\right) d y \\
& =\frac{1}{2}-\frac{1}{2} \sum_{l=1}^{L_{d}} \sum_{m=1}^{r_{l}} \frac{B_{l m}}{(m-1) ! \lambda_{l}^{m}} \cdot \int_{\alpha_{0}^{2}}^{\infty} \operatorname{erf}\left(\sqrt{\frac{E_{b} y}{N_{0}}}\right) \cdot\left(y-\alpha_{0}^{2}\right)^{m-1} \exp \left(-\frac{\left(y-\alpha_{0}^{2}\right)}{\lambda_{l}}\right) d y
\end{aligned}
$$

Substituting the integral expression in the above equation for $I$

$$
I=\int_{\alpha_{0}^{2}}^{\infty} u d v
$$

where

$$
\begin{aligned}
& u=\operatorname{erf}\left(\sqrt{\frac{E_{b} y}{N_{0}}}\right)=\frac{2}{\sqrt{\pi}} \int_{0}^{\sqrt{\frac{E_{b} y}{N_{0}}}} \exp \left(-t^{2}\right) d t \\
& d v=\left(y-\alpha_{0}^{2}\right)^{m-1} \exp \left(-\frac{\left(y-\alpha_{0}^{2}\right)}{\lambda_{l}}\right) d y
\end{aligned}
$$

which gives

$$
\begin{gathered}
d u=\sqrt{\frac{E_{b}}{N_{0} \pi}} \exp \left(-\frac{E_{b} y}{N_{0}}\right) y^{-1 / 2} d y \\
v=-\sum_{n=0}^{m-1} \frac{(m-1) ! \lambda_{l}^{n+1}}{(m-n-1) !}\left(y-\alpha_{0}^{2}\right)^{m-n-1} \exp \left(-\frac{y-\alpha_{0}^{2}}{\lambda_{l}}\right) .
\end{gathered}
$$

Integrating (C.2) by parts yields 


$$
\begin{aligned}
I= & {\left[-\operatorname{erf}\left(\sqrt{\frac{E_{b} y}{N_{0}}}\right)_{n=0}^{m-1} \frac{(m-1) ! \lambda_{l}^{n+1}}{(m-n-1) !}\left(y-\alpha_{0}^{2}\right)^{m-n-1} \exp \left(-\frac{y-\alpha_{0}^{2}}{\lambda_{l}}\right)\right]_{y=\alpha_{0}^{2}}^{\infty} } \\
& +\sqrt{\frac{E_{b}}{N_{0} \pi}} \sum_{n=0}^{m-1} \frac{(m-1) ! \lambda_{l}^{n+1}}{(m-n-1) !} \exp \left(\frac{\alpha_{0}^{2}}{\lambda_{l}}\right) \int_{\alpha_{0}^{2}}^{\infty} \frac{\left(y-\alpha_{0}^{2}\right)^{m-n-1}}{\sqrt{y}} \exp \left[-\left(\frac{E_{b}}{N_{0}}+\frac{1}{\lambda_{l}}\right) y\right] d y
\end{aligned}
$$

From [23] we obtain the following expression for the integral term in (C.7)

$$
\int_{\alpha_{0}^{2}}^{\infty} \frac{\left(y-\alpha_{0}^{2}\right)^{\mu-1}}{\sqrt{y}} e^{-C_{l} y} d y=C_{l}^{-\frac{\left(\mu+\frac{1}{2}\right)}{2}} \alpha_{0}^{\left(\mu-\frac{3}{2}\right)} \Gamma(\mu) \exp \left(-\frac{C_{l} \alpha_{0}^{2}}{2}\right) W_{\frac{1}{2}-\mu} \frac{\frac{1}{2}-\mu}{2}\left(C_{l} \alpha_{0}^{2}\right)
$$

where $\mu=m-n, C_{l}=\frac{E_{b}}{N_{0}}+\frac{1}{\lambda_{l}}$ and $\Gamma(z)$ is the Gamma function. $W_{\lambda, v}(z)$ is known as the Whittaker function which is defined as [23]

$$
W_{\lambda, v}(z)=\frac{\Gamma(-2 v)}{\Gamma\left(\frac{1}{2}-v-\lambda\right)} M_{\lambda, v}(z)+\frac{\Gamma(2 v)}{\Gamma\left(\frac{1}{2}+v-\lambda\right)} M_{\lambda,-v}(z)
$$

where

$$
\begin{gathered}
M_{\lambda, v}(z)=z^{v+\frac{1}{2}} e^{-z / 2} \Phi\left(v-\lambda+\frac{1}{2}, 2 v+1 ; z\right) \\
M_{\lambda,-v}(z)=z^{-v+\frac{1}{2}} e^{-z / 2} \Phi\left(-v-\lambda+\frac{1}{2},-2 v+1 ; z\right)
\end{gathered}
$$

$\Phi(\alpha, \gamma ; z)$ is the confluent hypergeometric function [23]

$$
\Phi(\alpha, \gamma ; z)=1+\frac{\alpha z}{\gamma 1 !}+\frac{\alpha(\alpha+1) z^{2}}{\gamma(\gamma+1) 2 !}+\ldots
$$

Equation (C.7) thus simplifies to 


$$
\begin{aligned}
I & =\operatorname{erf}\left(\sqrt{\frac{E_{b} \alpha_{0}^{2}}{N_{0}}}\right)(m-1) ! \lambda_{l}^{m} \\
& +\sqrt{\frac{E_{b}}{N_{0} \pi}} \sum_{n=0}^{m-1} \frac{(m-1) ! \lambda_{l}^{n+1}}{(\mu-1) !} \exp \left[\left(\frac{1}{\lambda_{l}}-\frac{C_{l}}{2}\right) \alpha_{0}^{2}\right] C_{l}^{-\frac{\left(\mu+\frac{1}{2}\right)}{2}} \alpha_{0}^{\left(\mu-\frac{3}{2}\right)} \Gamma(\mu) W_{\frac{1}{2}-\mu} \frac{\frac{1}{2}-\mu}{2}\left(C_{l} \alpha_{0}^{2}\right)
\end{aligned}
$$

which results in the following expression for $P_{b}$,

$$
\begin{aligned}
& P_{b}=\frac{1}{2}-\frac{1}{2} \sum_{l=1}^{L_{d}} \sum_{m=1}^{r_{l}} \frac{B_{l m}}{(m-1) ! \lambda_{l}^{m}}\left[\operatorname{erf}\left(\sqrt{\frac{E_{b} \alpha_{0}^{2}}{N_{0}}}\right)(m-1) ! \lambda_{l}^{m}\right. \\
& \left.+\sqrt{\frac{E_{b}}{N_{0} \pi}} \sum_{n=0}^{m-1} \frac{(m-1) ! \lambda_{l}^{n+1}}{(\mu-1) !} \exp \left[\left(\frac{1}{\lambda_{l}}-\frac{C_{l}}{2}\right) \alpha_{0}^{2}\right] C_{l}^{-\frac{\left(\mu+\frac{1}{2}\right)}{2}} \alpha_{0}^{\left(\mu-\frac{3}{2}\right)} \Gamma(\mu) W_{\frac{1}{2}-\mu}, \frac{\frac{1}{2}-\mu}{2}\left(C_{l} \alpha_{0}^{2}\right)\right]^{-(\text {С. } \beta) ~}
\end{aligned}
$$

Equation (C.13) can be written in a more simplified form when $r_{l}=1, \forall l$

$$
P_{b}=\frac{1}{2}-\frac{1}{2} \sum_{l=1}^{L_{d}} B_{l 1}\left[\operatorname{erf}\left(\sqrt{\frac{E_{b} \alpha_{0}^{2}}{N_{0}}}\right)+\sqrt{\frac{E_{b}}{N_{0} \pi}} \exp \left(\frac{\alpha_{0}^{2}}{\lambda_{l}}\right) \sqrt{\frac{\pi}{C_{l}}} \operatorname{erfc}\left(\sqrt{C_{l} \alpha_{0}^{2}}\right)\right]
$$

where $\operatorname{erf}(x)$ and $\operatorname{erfc}(x)$ are the error and complementary error functions respectively. 


\section{Appendix D : Calculating the Errors in Amplitude and Phase estimation}

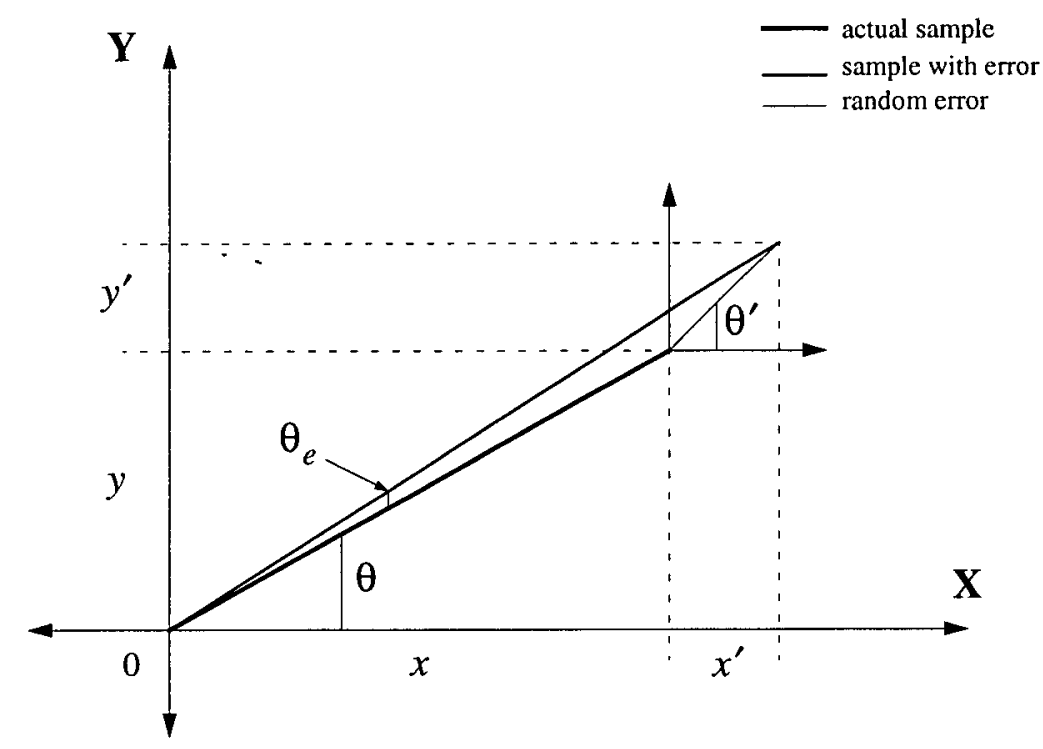

Figure D.1 Resolution of the fading sample with/without error

Figure D. 1 illustrates the resolution of the fading sample, $s=x+j y$, the error variable, $e=x^{\prime}+j y^{\prime}$ and the fading sample with error, $s_{e}=\left(x+x^{\prime}\right)+j\left(y+y^{\prime}\right)$ in the XY-plane. $x^{\prime}$ and $y^{\prime}$ are both i.i.d. Gaussian random variables with variance, $\sigma_{x^{\prime}}^{2}=\sigma_{y^{\prime}}^{2}=\sigma_{e}^{2 / 2}$ where $\sigma_{e}^{2}$ is the variance of $e$.

The amplitude estimation error is given by

$$
\varepsilon_{r}=\left|s_{e}\right|-|s|
$$

where $\left|s_{e}\right|=\sqrt{\left(x+x^{\prime}\right)^{2}+\left(y+y^{\prime}\right)^{2}}$ has a Rician distribution and $|s|=\sqrt{(x)^{2}+(y)^{2}}$ is a constant. The mean square error (MSE) is therefore a function of the amplitude of the fading sample and is given by 


$$
\begin{aligned}
E\left[\varepsilon_{r}^{2}\right] & =E\left[\left|s_{e}\right|^{2}+|s|^{2}-2\left|s_{e}\right||s|\right] \\
& =E\left[\left|s_{e}\right|^{2}\right]+|s|^{2}-2|s| E\left[\left|s_{e}\right|\right] \\
& =\sigma_{e}^{2}+2 \cdot|s|^{2}-\left(|s| \sqrt{\pi} \cdot \sqrt{\sigma_{e}^{2}} \exp \left(-\frac{|s|^{2}}{\sigma_{e}^{2}}\right) \Phi\left(\frac{3}{2}, 1 ; \frac{|s|^{2}}{\sigma_{e}^{2}}\right)\right)
\end{aligned}
$$

where $\Phi(a, b ; z)$ is the confluent hypergeometric function [23]. $E\left[\left|s_{e}\right|^{2}\right]$ and $E\left[\left|s_{e}\right|\right]$ are the first moments of the non-central chi squared and Rician distributed random variables respectively as shown in [12]. The normalized amplitude estimation error, $E_{N}$, is written as

$$
E_{N}=\frac{E\left[\varepsilon_{r}^{2}\right]}{|s|^{2}}
$$

Since the phase of the fading sample is uniform from 0 to $2 \pi$, the phase estimation error, $\theta_{e}$, is independent of $\theta$ and can be obtained by assuming $\theta=0$ in Figure D.1. It thus follows

$$
\theta_{e}=\operatorname{atan}\left(\frac{|e| \sin \theta^{\prime}}{|s|+|e| \cos \theta^{\prime}}\right)
$$

Letting $R=|e|$ and knowing from [19] the joint pdf, $f_{R \theta^{\prime}}(r, \varphi)$, of $R$ and $\theta^{\prime}$ which is given by

$$
f_{R \theta^{\prime}}(r, \varphi)=\frac{r}{\pi \sigma_{e}^{2}} \exp \left(-\frac{r^{2}}{\sigma_{e}^{2}}\right)
$$

the mean phase estimation error, $E\left[\theta_{e}\right]$, is obtained by integrating (D.4) over $f_{R \theta^{\prime}}(r, \varphi)$, i.e.

$$
E\left[\theta_{e}\right]=\frac{1}{\pi \sigma_{e}^{2}} \int_{0}^{2 \pi} \int_{0}^{\infty} r \operatorname{atan}\left(\frac{r \sin \varphi}{|s|+r \cos \varphi}\right) \exp \left(-\frac{r^{2}}{\sigma_{e}^{2}}\right) d r d \varphi
$$




\section{Bibliography}

[1] T. S. Rappaport, Wireless Communications Principles \& Practice. N.J.: Prentice Hall PTR, 1996.

[2] L. Litwin, “An introduction to multicarrier modulation," IEEE Potentials, pp. 36-38, Apr/ May 2000.

[3] R. J. C. Bultitude and G. K. Bedal, "Propagation characteristics on microcellular urban mobile radio channels at $910 \mathrm{MHz}$," IEEE Journal on Selected Areas in Comm., vol. 7, no. 1, pp. 31-39, Jan. 1989.

[4] W. C. Lindsey, "Error probabilities for Rician fading multichannel reception of binary and N-ary signals," IEEE Trans. Inform. Theory, pp. 339-350, Oct. 1964.

[5] J. E. Mazo, "Exact matched filter bound for two-beam Rayleigh fading," IEEE Trans. Comm., vol. 39, no. 7, pp. 1027-1030, Jul. 1991.

[6] M.V. Clark, L. J. Greenstein, W. K. Kennedy, and M. Shafi, "Matched filter performance bounds for diversity combining receivers in digital mobile radio," IEEE Trans. Veh. Tech., vol. 41, no. 4, pp. 356-362, Nov. 1992.

[7] K. W. Yip and T. S. Ng, "Matched filter bound for multipath Rician-fading channels," IEEE Trans. Comm., vol. 46, no. 4, pp. 441-445, Apr. 1998.

[8] W. Burchill and C. Leung, "Matched filter bound for OFDM on Rayleigh fading channels," Electronics Letters, vol. 31, no. 20, pp. 1716-1717, Sep. 1995.

[9] N. J. Baas and D. P. Taylor, "Matched filter bounds for wireless communication over Rayleigh fading dispersive channels," IEEE Trans. Comm., vol. 49, no. 9, pp. 1525-1528, Sept. 2001.

[10] R. Boudreau, J. Y. Chouinard and A. Yongacoglu, "Exploiting Doppler-diversity in flat, fast fading channels," 2000 Canadian Conference on Electrical and Computer Engineering, vol.1, pp. 270-274, 2000.

[11] B. Sklar, "Rayleigh fading channels in mobile digital communication systems part I: characterization," IEEE Comm. Mag., pp. 90-100, Jul. 1997.

[12] J. G. Proakis, Digital Communications. N.Y.: McGraw-Hill, 3rd Edition, 1995.

[13] D. C. Cox and R. P. Leck, "Distributions of multipath delay spread and average excess delay for $910 \mathrm{MHz}$ urban mobile radio paths," IEEE Trans. Antennas Propagat., vol. AP-23, pp. 206-213, Mar. 1975. 
[14] W. S. Burchill, Performance and Receiver Structures for OFDM on Rayleigh Fading Channels. Ph.D. thesis, Dept. of Elec. \& Comp. Eng., U.B.C., 1997.

[15] W. C. Jakes, Microwave Mobile Communications. N.Y.: Wiley, 1974.

[16] A. Papoulis, Probability, Random Variables, and Stochastic Processes. N.Y.: McGraw-Hill, 1984.

[17] R. H. Clarke, “A statistical theory of mobile-radio reception," Bell Syst. Tech. J., vol. 47, pp. 957-1000, 1968.

[18] S. Haykin, Communication Systems. N.Y.: Wiley, 3rd edition, 1999.

[19] W. B. Davenport, Jr. and W. L. Root, An Introduction to the Theory of Random Signals and Noise. N.Y.: McGraw-Hill, 1958.

[20] E. Kreyszig, Advanced Engineering Mathematics. N.Y.: Wiley, 8th edition, 1999.

[21] S. S. Haykin, Introduction to Adaptive Filters. N.Y.: MacMillan, 1984.

[22] The MathWorks, Inc., Matlab ${ }^{\circledR}$, ver. 6.1.0.450, release 12.1, May 2001.

[23] I. S. Gradshteyn, I. M. Ryzhik, Table of Integrals, Series and Products. London: Academic Press, 6th edition, 2000.

[24] D. J. Young and N. C. Beaulieu, "The generation of correlated Rayleigh random variates by inverse discrete Fourier transform," IEEE Trans. Comm., vol. 48, no. 7, pp. 1114-1127, Jul.2000.

[25] J. I. Smith, “A computer generated multipath fading simulation for mobile radio," IEEE Trans. Veh. Tech., vol. VT-24, no. 3, pp. 39-40, Aug. 1975.

[26] J. M. Wozencraft, I. M. Jacobs, Principles of Communication Engineering. N.Y.: Wiley, 1965.

[27] S. Kotz, N. L. Johnson, D. W. Boyd, "Series representations of distributions of quadratic forms in normal variables II. Non-central case," Annals of Mathematical Statistics, vol. 38, issue 3, pp. 838-848, Jun. 1967.

[28] M. Abramowitz and I.A. Stegun, Handbook of Mathematical functions. N.Y.: Dover Publications, Inc., 1965. 